\title{
HEISENBERG CATEGORIFICATION AND HILBERT SCHEMES
}

SABIN CAUTIS and ANTHONY LICATA

\begin{abstract}
Given a finite subgroup $\Gamma \subset \mathrm{SL}_{2}(\mathbb{C})$ we define an additive 2-category $\mathscr{H}_{\Gamma}$ whose Grothendieck group is isomorphic to an integral form $\mathfrak{h}_{\Gamma}$ of the Heisenberg algebra. We construct an action of $\mathscr{H}_{\Gamma}$ on derived categories of coherent sheaves on Hilbert schemes of points on the minimal resolutions $\overline{\mathbb{C}^{2} / \Gamma}$.
\end{abstract}

\section{Contents}

1. Introduction . . . . . . . . . . . . . . . . . . . . . . . . . . 2469

2. Quantum Heisenberg algebras . . . . . . . . . . . . . . . . 2472

3. The 2 -category $\mathscr{H}_{\Gamma} \ldots \ldots \ldots \ldots$

4. A 2-representation of $\mathscr{H}_{\Gamma} \ldots \ldots \ldots$

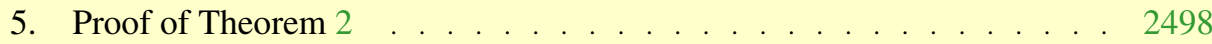

6. The alternate 2 -category $\mathscr{H}^{\Gamma} \ldots \ldots \ldots$

7. Proof of Theorem 1 . . . . . . . . . . . . . . . . . . . . . . . . . . . 2525

8. Relation to Hilbert schemes $\quad \ldots \quad \ldots \quad \ldots$

9. An abelian 2-representation of $\mathscr{H}_{\Gamma} \ldots \ldots \ldots$

10. Other remarks and open problems . . . . . . . . . . . . . . . . 2538

Appendix. The case $\Gamma=\mathbb{Z}_{2} \ldots \ldots \ldots \ldots$. . . . . . . . . . . . . . . . . . 2543

References . . . . . . . . . . . . . . . . . . . . 2545

\section{Introduction}

The well-documented relationship between the Heisenberg algebra and Hilbert schemes of points on a surface $S$ involves an action of the Heisenberg algebra on the cohomology $\bigoplus_{n} H^{*}\left(\operatorname{Hilb}^{n}(S)\right)$ (see [12], [19]). Inspired by other constructions from geometric representation theory, one expects this Heisenberg action on cohomology to lift to algebraic $K$-theory, though, in general, lifting this action is not completely straightforward (see [25] and [8] for $K$-theoretic constructions when the 
surface is $\mathbb{C}^{2}$ ). Of particular interest in representation theory is the case when the surface $S=\widehat{\mathbb{C}^{2} / \Gamma}$ is the minimal resolution of the quotient of $\mathbb{C}^{2}$ by a nontrivial finite subgroup $\Gamma \subset \mathrm{SL}_{2}(\mathbb{C})$; in this case one expects a Heisenberg action on $K$-theory to be the basic building block for a $K$-theoretic construction of the basic representation of the corresponding affine Lie algebra.

After $K$-theory, however, one can investigate yet another level of structure by considering the derived categories of coherent sheaves $\bigoplus_{n} D \operatorname{Coh}\left(\operatorname{Hilb}^{n}(S)\right)$ on these Hilbert schemes. Then one expects a Heisenberg algebra action on $K$-theory to be the shadow of a richer categorical Heisenberg action on these triangulated categories.

More precisely, the Hilbert schemes $\left\{\operatorname{Hilb}^{n}(S)\right\}_{n \in \mathbb{N}}$ naturally form a 2-category $\operatorname{Hilb}(S)$ :

(1) The objects of $\operatorname{Hilb}(S)$ are the natural numbers $\mathbb{N}$.

(2) The 1-morphisms from $m$ to $n$ are compactly supported objects in $D \mathrm{Coh}\left(\operatorname{Hilb}^{m}(S) \times \operatorname{Hilb}^{n}(S)\right)$. The composition of 1-morphisms is given by convolution.

(3) For $\mathcal{P}_{1}, \mathcal{P}_{2} \in D \operatorname{Coh}\left(\operatorname{Hilb}^{m}(S) \times \operatorname{Hilb}^{n}(S)\right)$, the space of 2-morphisms from $\mathcal{P}_{1}$ to $\mathcal{P}_{2}$ is the space $\operatorname{Ext}^{*}\left(\mathcal{P}_{1}, \mathcal{P}_{2}\right)$. The composition of 2-morphisms is the natural composition of Exts.

So to give a categorical Heisenberg action one should define both a Heisenberg 2category $\mathscr{H}_{S}$ and a 2 -functor $\mathscr{H}_{S} \rightarrow \operatorname{Hilb}(S)$. The Grothendieck group of $\mathscr{H}_{S}$ should be isomorphic to the Heisenberg algebra so that by passing to $K$-theory one gets a Heisenberg action on $\bigoplus_{n} K_{0}\left(\operatorname{Hilb}^{n}(S)\right)$. In this paper we construct such a 2-category $\mathscr{H}_{S}=\mathscr{H}_{\Gamma}$ and a 2-functor $\mathscr{H}_{\Gamma} \rightarrow \operatorname{Hilb}(S)$ when $S=\widehat{\mathbb{C}^{2} / \Gamma}$ is the minimal resolution of the quotient of $\mathbb{C}^{2}$ by a nontrivial finite subgroup $\Gamma \subset \mathrm{SL}_{2}(\mathbb{C})$ (Theorems 1 and 2). In particular, we obtain a (quantum) Heisenberg algebra action on $\bigoplus_{n} K_{0}\left(\operatorname{Hilb}^{n}(S)\right)$. (We also note here that in the special case when $S=\mathbb{C}^{2}$ a natural $\mathbb{C}^{\times}$-action together with localization techniques have already been used to define Heisenberg algebra actions on localized equivariant $K$-theory; see [8], [25]).

\subsection{Analogy with Kac-Moody Lie algebra actions}

There are clear parallels between our story for Heisenberg algebras $\mathfrak{h}_{\Gamma}$ and the categorification of Kac-Moody algebras $U(\mathfrak{g})$. In [20] and [22], Nakajima constructed actions of the enveloping algebra $U(\mathfrak{g})$ on the cohomology and $K$-theory of quiver varieties, generalizing earlier work of Ginzburg for $U\left(\mathfrak{s l}_{n}\right)$ (see [7]). On the other hand, in [15], [16], and [17], Khovanov and Lauda define a 2-category $U(\mathfrak{g})$ whose Grothendieck group is isomorphic to (an integral version of) the quantized enveloping algebra. There is also independent and very similar work of Rouquier [24] in the same direction. 
In [5], the authors, together with Joel Kamnitzer, construct a "geometric categorical $\mathfrak{g}$ action" on the derived category of coherent sheaves on quiver varieties. This action conjecturally induces an action of the 2-categories $\mathcal{U}(\mathfrak{g})$ on the derived categories of quiver varieties. The 2-categories $\mathcal{U}(\mathfrak{g})$ and these actions on the derived categories of quiver varieties are analogous to our Heisenberg 2-categories $\mathscr{H}_{\Gamma}$ and their action on the derived categories of Hilbert schemes.

\subsection{The 2-category $\mathscr{H}_{\Gamma}$}

By the McKay correspondence, isomorphism classes of finite subgroups $\Gamma \subset \mathrm{SL}_{2}(\mathbb{C})$ are parameterized by simply-laced affine Dynkin diagrams (i.e., diagrams of type $\widehat{A}_{n}$, $\widehat{D}_{n}, \widehat{E}_{6}, \widehat{E}_{7}$, and $\widehat{E}_{8}$ ). Each of these affine Dynkin diagrams, in turn, gives rise to a presentation for a Heisenberg algebra $\mathfrak{h}_{\Gamma}$, which we describe in Section 2.2, and a presentation of a 2-category $\mathscr{H}_{\Gamma}$, which we define in Section 3. The 2-morphisms in $\mathscr{H}_{\Gamma}$ are defined using a planar graphical calculus reminiscent of the graphical calculus used by Khovanov and Lauda [16] in their categorification of quantum groups. Our first main theorem (Theorem 1$)$ says that the Grothendieck group $K_{0}\left(\mathscr{H}_{\Gamma}\right)$ is isomorphic to the Heisenberg algebra $\mathfrak{h}_{\Gamma}$. In particular, the indecomposable 1-morphisms of $\mathcal{H}_{\Gamma}$ descend to a "canonical basis" of the Heisenberg algebra.

\subsection{Actions of $\mathscr{H}_{\Gamma}$}

A direct relationship between the finite group $\Gamma \subset \mathrm{SL}_{2}(\mathbb{C})$ and the Heisenberg algebra $\mathfrak{h}_{\Gamma}$ can be formulated in two closely related ways (one algebraic and the other geometric). In the algebraic setting one can consider the wreath products $\Gamma^{n} \rtimes S_{n}$ of $\Gamma$ with the symmetric group $S_{n}$. Then, following [9], one constructs the basic representation of $\mathfrak{h}_{\Gamma}$ on the Grothendieck groups of the module categories $\mathbb{C}\left[\Gamma^{n} \rtimes S_{n}\right]-$ mod. Geometrically, following [19] and [12], one considers the Hilbert schemes $\operatorname{Hilb}^{n}\left(\widehat{\mathbb{C}^{2} / \Gamma}\right)$ and constructs a representation of $\mathfrak{h}_{\Gamma}$ on their cohomology.

We will work in a setting that shares features with both the algebraic and geometric constructions above by considering directly the bounded derived categories $D\left(A_{n}^{\Gamma}-\mathrm{gmod}\right)$ of finitely generated graded $A_{n}^{\Gamma}$-modules. Here $A_{n}^{\Gamma}$ is the algebra

$$
A_{n}^{\Gamma}:=\left[\left(\operatorname{Sym}^{*}\left(V^{\vee}\right) \rtimes \Gamma\right) \otimes\left(\operatorname{Sym}^{*}\left(V^{\vee}\right) \rtimes \Gamma\right) \otimes \cdots \otimes\left(\operatorname{Sym}^{*}\left(V^{\vee}\right) \rtimes \Gamma\right)\right] \rtimes S_{n},
$$

which inherits the natural grading from $\operatorname{Sym}^{*}\left(V^{\vee}\right)$ (here $\left.V=\mathbb{C}^{2}\right)$.

We recall why $D\left(A_{n}^{\Gamma}-\mathrm{gmod}\right)$ is equivalent to derived categories of Hilbert schemes in Section 8 and explain the relationship to $\mathbb{C}\left[\Gamma^{n} \rtimes S_{n}\right]-$ mod in Section 9.6. Our second main theorem (Theorem 2) constructs a natural action of the Heisenberg

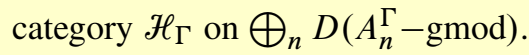

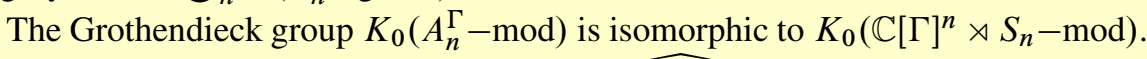
On the other hand, $K_{0}\left(A_{n}^{\Gamma}-\mathrm{mod}\right) \cong K_{0}\left(\mathrm{Hilb}^{n}\left(\widehat{\mathbb{C}^{2} / \Gamma}\right)\right)$ is isomorphic to the coho- 
mology of $\operatorname{Hilb}^{n}\left(\widehat{\mathbb{C}^{2} / \Gamma}\right)$. Thus the category $\bigoplus_{n} D\left(A_{n}^{\Gamma}-\right.$ mod $)$ gives a common categorification of the spaces used by Nakajima, Grojnowski, and Frenkel, Jing, and Wang (see [9], [10], [12], and [19]).

\subsection{Organization}

- $\quad$ Section 2 defines the (quantum) Heisenberg algebra $\mathfrak{h}_{\Gamma}$. Our choice of generators for $\mathfrak{h}_{\Gamma}$ differs somewhat from the choices made elsewhere in the literature.

- $\quad$ Section 3 defines the 2-category $\mathscr{H}_{\Gamma}$ which categorifies $\mathfrak{h}_{\Gamma}$. We also define a map from $\mathfrak{h}_{\Gamma}$ to the Grothendieck group of $\mathscr{H}_{\Gamma}$. This map is shown to be an isomorphism in Section 7 (Theorem 1).

- Sections 4 and 5 are concerned with defining an action of $\mathscr{H}_{\Gamma}$ on $\bigoplus_{n} D\left(A_{n}^{\Gamma}-\mathrm{mod}\right)$.

- In Section 6 we study a slightly different 2-category $\mathscr{H}^{\Gamma}$ using an alternative simplified graphical calculus. $\mathscr{H}^{\Gamma}$ is "Morita equivalent" to $\mathscr{H}_{\Gamma}$ in the sense that the spaces of 2-morphisms in $\mathscr{H}^{\Gamma}$ and $\mathscr{H}_{\Gamma}$ are Morita equivalent. (Subsequently, the 2-representation theories of $\mathscr{H}^{\Gamma}$ and $\mathscr{H}_{\Gamma}$ are equivalent.) We construct a natural functor $\eta: \mathscr{H}^{\Gamma} \rightarrow \mathscr{H}_{\Gamma}$, which induces an isomorphism at the level of Grothendieck groups. (So both $\mathscr{H}^{\Gamma}$ and $\mathscr{H}_{\Gamma}$ categorify $\mathfrak{h}_{\Gamma}$.) The 2category $\mathscr{H}^{\Gamma}$ is of independent interest but another motivation for introducing it is to facilitate the graphical computations needed to prove Theorem 1.

- In Section 9 we sketch a second, simpler (nonderived) action of $\mathscr{H}_{\Gamma}$, related to the action of Section 4 by Koszul duality.

- Section 10 contains a discussion of currently unanswered questions and further directions.

- $\quad$ Appendix: most of the paper assumes that the Dynkin diagram associated to $\Gamma \subset \mathrm{SL}_{2}(\mathbb{C})$ is simply-laced, which simplifies notation but fails to cover the case $\Gamma=\mathbb{Z}_{2}$. In the appendix we collect the required definitions for $\Gamma=\mathbb{Z}_{2}$. (With these modifications the theorems of the paper hold for any nontrivial $\Gamma$.) The definition of the Heisenberg category for the trivial subgroup of $\mathrm{SL}_{2}(\mathbb{C})$ is of interest in its own right, but is not discussed directly in this paper.

\section{Quantum Heisenberg algebras}

\subsection{The McKay correspondence}

Fix an algebraically closed field $\mathbb{k}$ of characteristic zero. Let $\Gamma \subset \mathrm{SL}_{2}(\mathbb{k})$ denote a finite subgroup. (In our discussion this can include $\Gamma=\mathbb{k}^{\times}$, the Cartan subgroup of diagonal matrices.) For notational convenience, the body of the paper assumes that $\Gamma \neq \mathbb{Z}_{2}$. We deal with this extra case in the appendix. 
Denote by $V$ the standard two-dimensional representation of $\Gamma$. Under the McKay correspondence, the finite subgroup $\Gamma$ corresponds to an affine Dynkin diagram with vertex set $I_{\Gamma}$ and edge set $E_{\Gamma}$. By definition, each vertex $i \in I_{\Gamma}$ is indexed by an irreducible representation $V_{i}$ of $\Gamma$ and two vertices $i, j \in I_{\Gamma}$ are joined by $k$ edges, where $k$ is the number of times $V_{j}$ appears as a direct summand of $V_{i} \otimes V$. Notice that since $V$ is self-dual this relation is symmetric in $i$ and $j$.

For instance, when $G=\mathbb{Z} / n \mathbb{Z}$ we get the affine Dynkin diagram $\widehat{A_{n-1}}$, which is an $n$ cycle. When $\Gamma=\mathbb{k}^{\times}$, the associated diagram is the Dynkin diagram $A_{\infty}$ of the Lie algebra $\mathfrak{s l}_{\infty}$.

Define the quantum Cartan matrix $C_{\Gamma}$ to be the matrix with entries $\langle i, j\rangle$ indexed by $i, j \in I_{\Gamma}$ given by

$$
\langle i, j\rangle= \begin{cases}t+t^{-1} & \text { if } i=j, \\ -1 & \text { if } i \neq j \text { are connected by an edge } \\ 0 & \text { if } i \neq j \text { are not connected by an edge. }\end{cases}
$$

Note that at $t=1$ the matrix $C_{\Gamma}$ becomes the extended Cartan matrix of type ADE. (This is the McKay correspondence between nontrivial finite subgroups $\Gamma \subset \mathrm{SL}_{2}(\mathbb{C})$ and simply-laced affine Dynkin diagrams.)

\subsection{The quantum Heisenberg algebra associated to $\Gamma$}

We define the Heisenberg algebra $\mathfrak{h}_{\Gamma}$ associated to $\Gamma$ to be the unital $\mathbb{k}\left[t, t^{-1}\right]$-algebra with generators $p_{i}^{(n)}, q_{i}^{(n)}$ for $i \in I_{\Gamma}$ and integers $n \geq 0$ and relations

$$
\begin{aligned}
& p_{i}^{(n)} p_{j}^{(m)}=p_{j}^{(m)} p_{i}^{(n)} \quad \text { for all } i, j \in I_{\Gamma}, \\
& q_{i}^{(n)} q_{j}^{(m)}=q_{j}^{(m)} q_{i}^{(n)} \quad \text { for all } i, j \in I_{\Gamma}, \\
& q_{i}^{(n)} p_{i}^{(m)}=\sum_{k \geq 0}[k+1] p_{i}^{(m-k)} q_{i}^{(n-k)} \quad \text { for all } i \in I_{\Gamma}, \\
& q_{i}^{(n)} p_{j}^{(m)}=p_{j}^{(m)} q_{i}^{(n)}+p_{j}^{(m-1)} q_{i}^{(n-1)} \quad \text { for all } i \neq j \in I_{\Gamma} \text { with }\langle i, j\rangle=-1, \\
& q_{i}^{(n)} p_{j}^{(m)}=p_{j}^{(m)} q_{i}^{(n)} \quad \text { for all } i \neq j \in I_{\Gamma} \text { with }\langle i, j\rangle=0 .
\end{aligned}
$$

Here $[k+1]=t^{-k}+t^{-k+2}+\cdots+t^{k-2}+t^{k}$ denotes the quantum integer, and in the above relations we have set $p_{i}^{(0)}=q_{j}^{(0)}=1$ and $p_{i}^{(k)}=q_{i}^{(k)}=0$ when $k<0$. (Thus the summations in the above relations are all finite.)

\subsubsection{The usual Heisenberg presentation}

Most mathematical literature about the Heisenberg algebra uses a slightly different presentation than the one above. In level 1, which is the case of interest for this paper, 
the standard presentation of the Heisenberg algebra is as a unital $\mathbb{k}\left[t, t^{-1}\right]$-algebra generated by $a_{i}(n)$, for $i \in I_{\Gamma}$ and $n \in \mathbb{Z}-0$. The relations are

$$
\left[a_{i}(m), a_{j}(n)\right]=\delta_{m,-n}[n\langle i, j\rangle] \frac{[n]}{n} .
$$

Since the Dynkin diagram with vertex set $I_{\Gamma}$ is of affine type, this algebra is sometimes called the quantum toroidal Heisenberg algebra (since, in this case, the Heisenberg algebra is a subalgebra of the corresponding level 1 quantum toroidal algebra). By omitting the affine node from consideration, one recovers a smaller Heisenberg algebra, which is a subalgebra of the corresponding level 1 quantum affine algebra.

\section{Remark 1}

Sometimes in the literature, relation (6) above appears with a minus sign on the righthand side. However, this change of presentation does not alter the isomorphism type of the algebra, since replacing $a_{i}(m)$ by $-a_{i}(m)$ for $m>0$ takes one presentation to the other.

As will be shown in Lemma 1 below, the relationship between the generators $a_{i}(m)$ and $p_{i}^{(m)}, q_{i}^{(m)}$ is given by the following generating functions:

$$
\exp \left(\sum_{m \geq 1} \frac{a_{i}(-m)}{[m]} z^{m}\right)=\sum_{n \geq 0} p_{i}^{(n)} z^{n} \quad \text { and } \quad \exp \left(\sum_{m \geq 1} \frac{a_{i}(m)}{[m]} z^{m}\right)=\sum_{n \geq 0} q_{i}^{(n)} z^{n} .
$$

For example, comparing the first few coefficients gives the following equalities:

$$
\begin{aligned}
& p_{i}^{(0)}=1=q_{i}^{(0)}, \\
& p_{i}^{(1)}=a_{i}(-1), \quad q_{i}^{(1)}=a_{i}(1), \\
& p_{i}^{(2)}=\frac{1}{[2]} a_{i}(-2)+\frac{1}{2} a_{i}(-1)^{2}, \quad q_{i}^{(2)}=\frac{1}{[2]} a_{i}(2)+\frac{1}{2} a_{i}(1)^{2}, \quad \text { and so on. }
\end{aligned}
$$

The expressions in Equation 7 are known as "halves of vertex operators" and they appear naturally in vertex operator constructions of representation of quantum affine and toroidal algebras. For our categorical considerations, we find the generators $p_{i}^{(n)}, q_{i}^{(n)}$ more natural than the generators $a_{i}(m)$.

\section{LEMMA 1}

Relation (6) corresponds to relations (1)-(5) under the identification given by the vertex generating functions (7). 


\section{Proof}

We prove relations (3) and (4) above, the others being completely straightforward to check.

To prove relation (3), let us denote

$$
A(z):=\sum_{\ell \geq 1} \frac{a_{i}(-\ell)}{[\ell]} z^{\ell} \quad \text { and } \quad B(w):=\sum_{\ell \geq 1} \frac{a_{i}(\ell)}{[\ell]} w^{\ell} .
$$

Then $q_{i}^{(n)} p_{i}^{(m)}=\left[z^{m} w^{n}\right] \exp (B) \exp (A)$, where $\left[z^{m} w^{n}\right]$ means taking the coefficient of the polynomial $z^{m} w^{n}$ in the subsequent power series. Now, using relation (6), it follows that

$$
\begin{aligned}
{[A, B] } & =\sum_{\ell \geq 1} \frac{[2 \ell]}{\ell[\ell]} w^{\ell} z^{\ell} \\
& =\sum_{\ell \geq 1} \frac{\left(t^{\ell}+t^{-\ell}\right)}{\ell} w^{\ell} z^{\ell} \\
& =\log \left((1-t w z)\left(1-t^{-1} w z\right)\right) .
\end{aligned}
$$

It follows that $[A, B]$ commutes with $A$ and $B$ and hence that

$$
\exp (B) \exp (A)=\exp (-[A, B]) \exp (A) \exp (B)
$$

(see, e.g., [21, Lemma 9.43]). Thus

$$
\begin{aligned}
{\left[z^{m} w^{n}\right] \exp (B) \exp (A) } & =\left[z^{m} w^{n}\right] \frac{1}{(1-t w z)\left(1-t^{-1} w z\right)} \exp (A) \exp (B) \\
& =\left[z^{m} w^{n}\right] \sum_{\ell \geq 0}[\ell+1](w z)^{\ell} \exp (A) \exp (B) \\
& =\sum_{k \geq 0}[k+1] p_{i}^{(m-k)} q_{i}^{(n-k)},
\end{aligned}
$$

which is what we needed to prove.

In the case of relation (4), let us denote

$$
A(z):=\sum_{\ell \geq 1} \frac{a_{j}(-\ell)}{[\ell]} z^{\ell} \quad \text { and } \quad B(w):=\sum_{\ell \geq 1} \frac{a_{i}(\ell)}{[\ell]} w^{\ell} .
$$

Then $q_{i}^{(n)} p_{j}^{(m)}=\left[z^{m} w^{n}\right] \exp (B) \exp (A)$. However, this time

$$
[A, B]=\sum_{\ell \geq 1} \frac{[-1]}{\ell} w^{\ell} z^{\ell}=-\log (1-w z)
$$


and so

$$
\begin{aligned}
{\left[z^{m} w^{n}\right] \exp (B) \exp (A) } & =\left[z^{m} w^{n}\right](1-w z) \exp (A) \exp (B) \\
& =p_{j}^{(m)} q_{i}^{(n)}+p_{j}^{(m-1)} q_{i}^{(n-1)},
\end{aligned}
$$

which is what we needed to prove.

\subsubsection{The transposed generators}

There is an alternative generating set of $\mathfrak{h}_{\Gamma}$ given by elements $p_{i}^{\left(1^{n}\right)}$ and $a_{i}^{\left(1^{n}\right)}$. These are defined by halves of vertex operators which are similar to those of (7):

$$
\begin{aligned}
\exp \left(-\sum_{m \geq 1} \frac{a_{i}(-m)}{[m]} z^{m}\right) & =\sum_{n \geq 0}(-1)^{n} p_{i}^{\left(1^{n}\right)} z^{n} \quad \text { and } \\
\exp \left(-\sum_{m \geq 1} \frac{a_{i}(m)}{[m]} z^{m}\right) & =\sum_{n \geq 0}(-1)^{n} q_{i}^{\left(1^{n}\right)} z^{n} .
\end{aligned}
$$

Thus, for example,

$p_{i}^{\left(1^{0}\right)}=q_{i}^{\left(1^{0}\right)}=1$,

$p_{i}^{\left(1^{1}\right)}=a_{i}(-1), \quad q_{i}^{\left(1^{1}\right)}=a_{i}(1)$,

$p_{i}^{\left(1^{2}\right)}=-\frac{1}{[2]} a_{i}(-2)+\frac{1}{2} a_{i}(-1)^{2}, \quad q_{i}^{\left(1^{2}\right)}=-\frac{1}{[2]} a_{i}(2)+\frac{1}{2} a_{i}(1)^{2}, \quad$ and so on.

The commutation relations among the $p_{i}^{\left(1^{n}\right)}$ and $q_{i}^{\left(1^{n}\right)}$ are the same as those between the $p_{i}^{(n)}$ and $q_{i}^{(n)}$. (Just replace $(n)$ by $\left(1^{n}\right)$ everywhere.) These relations can be proven directly by arguments similar to those of Lemma 1, though they also follow from the existence of an automorphism $\psi$ defined in Section 2.2.3.

Perhaps more interesting are the following relations:

$$
\begin{aligned}
p_{i}^{(m)} p_{j}^{\left(1^{n}\right)} & =p_{j}^{\left(1^{n}\right)} p_{i}^{(m)} \quad \text { and } \quad q_{i}^{(m)} q_{j}^{\left(1^{n}\right)}=q_{j}^{\left(1^{n}\right)} q_{i}^{(m)}, \\
q_{i}^{\left(1^{m}\right)} p_{j}^{(n)} & = \begin{cases}p_{i}^{(n)} q_{i}^{\left(1^{m}\right)}+[2] p_{i}^{(n-1)} q_{i}^{\left(1^{m-1}\right)}+p_{i}^{(n-2)} q_{i}^{\left(1^{m-2}\right)} & \text { if } i=j, \\
\sum_{k \geq 0} p_{j}^{(n-k)} q_{i}^{\left(1^{m-k}\right)} & \text { if }\langle i, j\rangle=-1, \\
p_{j}^{(n)} q_{i}^{\left(1^{m}\right)} & \text { if }\langle i, j\rangle=0 .\end{cases}
\end{aligned}
$$

These relations can be checked directly in the same way as in the proof of Lemma 1.

\subsubsection{The automorphism $\psi$}

The Heisenberg algebra $\mathfrak{h}_{\Gamma}$ has an involutive automorphism: 


$$
\psi: \mathfrak{h}_{\Gamma} \longrightarrow \mathfrak{h}_{\Gamma}, \quad a_{i}(k) \mapsto(-1)^{k+1} a_{i}(k) .
$$

In terms of the alternative generators $p_{i}^{(m)}, q_{i}^{(m)}, p_{j}^{\left(1^{m}\right)}, q_{i}^{\left(1^{m}\right)}$, this has the effect of exchanging $(n)$ and $\left(1^{n}\right)$ :

$$
\psi: p_{i}^{(n)} \leftrightarrow p_{i}^{\left(1^{n}\right)}, \quad q_{i}^{(n)} \leftrightarrow q_{i}^{\left(1^{n}\right)} .
$$

\subsubsection{Idempotent modification}

The version of the Heisenberg algebra we use is actually an idempotent modification of $\mathfrak{h}_{\Gamma}$. (This is similar to the appearance of Lusztig's idempotent version of $U_{q}(\mathfrak{g})$ in the categorification of quantum groups; see [15]-[17], [24].) In the idempotent modification, the unit 1 is replaced by a collection of orthogonal idempotents $\left\{1_{m}\right\}_{m \in \mathbb{Z}}$, with

$$
1_{k+m} p_{i}^{(m)}=1_{k+m} p_{i}^{(m)} 1_{k}=p_{i}^{(m)} 1_{k}
$$

and

$$
1_{k-m} q_{i}^{(m)}=1_{k-m} q_{i}^{(m)} 1_{k}=q_{i}^{(m)} 1_{k} .
$$

The remaining defining relations in the unital algebra $h_{\Gamma}$ give, for each $k \in \mathbb{Z}$, a defining relation of the idempotent modification. Namely, take the original relation and add the idempotent $1_{k}$ at the ends of the left- and right-hand sides; for example,

$$
q_{i}^{(n)} p_{j}^{(m)} 1_{k}=p_{j}^{(m)} q_{i}^{(n)} 1_{k} \quad \text { for all } i \neq j \in I_{\Gamma} \text { with }\langle i, j\rangle=0 \text { and all } k \in \mathbb{Z} .
$$

Note that these relations do not depend essentially on the particular idempotent $1_{k}$. As a result, we abuse notation slightly and also denote both the unital algebra and its idempotent modification by $\mathfrak{h}_{\Gamma}$.

One feature of the idempotent modified $\mathfrak{h}_{\Gamma}$ is that it is a $\mathbb{k}\left[t, t^{-1}\right]$-linear category. (Just as any $\mathbb{k}$-algebra with a collection of idempotents is a $\mathbb{k}$-linear category.) The objects of this category are then the integers, while the space of morphisms from $n$ to $m$ is the $\mathbb{k}\left[t, t^{-1}\right]$-module $1_{n} \mathfrak{h}_{\Gamma} 1_{m}$. Since $\mathfrak{h}_{\Gamma}$ is already a category, its categorification $\mathscr{H}_{\Gamma}$ defined in Section 3 will be a 2-category.

\subsection{The Fock space}

Let $\mathfrak{h}_{\Gamma}^{-} \subset \mathfrak{h}_{\Gamma}$ denote the subalgebra generated by the $q_{i}^{(n)} 1_{k}$ for all $i \in I_{\Gamma}, k \leq 0$, and $n \geq 0$. Let $\operatorname{triv}_{0}$ denote the trivial representation of $\mathfrak{h}_{\Gamma}^{-}$, where $1_{0}$ acts as the identity and $1_{k}$ acts by 0 for $k<0$. The $\mathfrak{h}_{\Gamma}$-module $\mathcal{F}_{\Gamma}=\operatorname{Ind}_{\mathfrak{h}} \frac{\mathfrak{h}_{\Gamma}}{\Gamma}\left(\operatorname{triv}_{0}\right)$ given by inducing the trivial representation from $\mathfrak{h}_{\Gamma}^{-}$to $\mathfrak{h}_{\Gamma}$ is called the Fock space representation of $\mathfrak{h}_{\Gamma}$.

The Fock space $\mathcal{F}_{\Gamma}$ is naturally isomorphic to the space of polynomials in the commuting variables $\left\{p_{j}^{(m)}\right\}_{j \in I_{\Gamma}, m \geq 0}$. If we grade the Fock space by declaring 
$\operatorname{deg}\left(p_{j}^{(m)}\right)=m$, then the idempotent $1_{l} \in \mathfrak{h}_{\Gamma}$ acts by projecting onto the degree $l$ subspace inside $\mathcal{F}_{\Gamma} \cong \mathbb{k}\left[\left\{p_{j}^{(m)}\right\}_{j, m}\right]$. (In particular, when $l<0$ the idempotent $1_{l}$ acts by 0 .) We will construct categorifications of this representation in Sections 4 and 9.

\section{The 2-category $\mathscr{H}_{\Gamma}$}

\subsection{The algebra $B^{\Gamma}$}

To define the 2-category $\mathscr{H}_{\Gamma}$ which categorifies $\mathfrak{h}_{\Gamma}$ we first need to define the algebra $B^{\Gamma}$ and fix notation involving idempotents in this algebra.

Since $\Gamma$ acts on $V$, it also acts on the exterior algebra $\Lambda^{*}(V)$. Let $B^{\Gamma}:=$ $\Lambda^{*}(V) \rtimes \Gamma$ be their semidirect product which contains both $\Lambda^{*}(V)$ and $\mathbb{k}[\Gamma]$ as subalgebras. This semidirect product is also called the smash product of $\mathbb{k}[\Gamma]$ and $\Lambda^{*}(V)$ and is sometimes denoted $\Lambda^{*}(V) \# \mathbb{k}[\Gamma]$ in the Hopf algebra literature, though we prefer the term semidirect product and avoid the \# notation.

Explicitly, an element in $B^{\Gamma}$ is a linear combination of terms $(v, \gamma)$, where $v \in$ $\Lambda^{*}(V)$ and $\gamma \in \Gamma$. The multiplication in $B^{\Gamma}$ is given by

$$
(v, \gamma) \cdot\left(v^{\prime}, \gamma^{\prime}\right)=\left(v \wedge\left(\gamma \cdot v^{\prime}\right), \gamma \gamma^{\prime}\right) .
$$

The natural $\mathbb{Z}$-grading on $\Lambda^{*}(V)$ extends to a grading of $B^{\Gamma}$ by putting $\mathbb{k}[\Gamma] \subset B^{\Gamma}$ in degree 0 . This makes $B^{\Gamma}$ into a superalgebra. We denote the degree of a homogeneous element $b \in B^{\Gamma}$ by $|b|$.

If we fix a basis $\left\{v_{1}, v_{2}\right\}$ of $V$ and let $\omega:=v_{1} \wedge v_{2} \in \wedge^{2}(V)$, then $B^{\Gamma}$ has a homogeneous basis over $\mathbb{k}$ given by $\left\{(1, \gamma),\left(v_{1}, \gamma\right),\left(v_{2}, \gamma\right),(\omega, \gamma)\right\}_{\gamma \in \Gamma}$.

Define a $\mathbb{k}$-linear trace $\operatorname{tr}: B^{\Gamma} \longrightarrow \mathbb{k}$ by setting

$$
\operatorname{tr}((\omega, \gamma))=\delta_{\gamma, 1} 1 \quad \text { and } \quad \operatorname{tr}((1, \gamma))=\operatorname{tr}\left(\left(v_{1}, \gamma\right)\right)=\operatorname{tr}\left(\left(v_{2}, \gamma\right)\right)=0 .
$$

The trace tr is supersymmetric (for any $a, b \in B^{\Gamma}$ we have $\operatorname{tr}(a b)=(-1)^{|a||b|} \operatorname{tr}(b a)$ ) and nondegenerate. This also induces a trace on $\mathbb{k}[\Gamma]$ via $\operatorname{tr}(\gamma):=\operatorname{tr}((\omega, \gamma))$. This corresponds to the usual trace on $\mathbb{k}[\Gamma]$ divided by $|\Gamma|$. (In this way $\operatorname{tr}(1)=1$.)

For a fixed $\mathbb{k}$-basis $\mathcal{B}$ of $B^{\Gamma}$, let $\mathscr{B}^{\vee}$ denote the basis of $B^{\Gamma}$ dual to $\mathscr{B}$ with respect to the associated nondegenerate bilinear form $\langle a, b\rangle:=\operatorname{tr}(a b)$. We denote the dual vector of $b \in \mathscr{B}$ by $b^{\vee} \in \mathscr{B}^{\vee}$.

\section{Remark 2}

We may think of elements of $B^{\Gamma}$ as $\mathbb{k}[\Gamma]$-module homomorphisms:

$$
B^{\Gamma} \cong \operatorname{Hom}_{\mathbb{k}[\Gamma]}\left(\mathbb{k}[\Gamma], \Lambda^{*}(V) \otimes \mathbb{k}[\Gamma]\right) .
$$

The above isomorphism is given by the following composition: 


$$
\begin{aligned}
B^{\Gamma} & \cong \operatorname{Hom}_{B \Gamma}\left(B^{\Gamma}, B^{\Gamma}\right) \cong \operatorname{Hom}_{B^{\Gamma}}\left(\operatorname{Ind}_{\mathbb{k}[\Gamma]}^{B^{\Gamma}} \mathbb{k}[\Gamma], B^{\Gamma}\right) \cong \operatorname{Hom}_{\mathbb{k}[\Gamma]}\left(\mathbb{k}[\Gamma], \operatorname{Res}_{B^{\Gamma}}^{\mathbb{k}[\Gamma]} B^{\Gamma}\right) \\
& \cong \operatorname{Hom}_{\mathbb{k}[\Gamma]}\left(\mathbb{k}[\Gamma], \Lambda^{*}(V) \otimes \mathbb{k}[\Gamma]\right) .
\end{aligned}
$$

\subsubsection{Idempotents}

Let $V_{1}, \ldots, V_{m}$ denote the distinct irreducible representations of $\Gamma$. By Maschke's theorem, the group algebra $\mathbb{k}[\Gamma]$ decomposes as a direct product of matrix algebras

$$
\mathbb{k}[\Gamma] \cong M_{n_{1}}(\mathbb{k}) \times \cdots \times M_{n_{m}}(\mathbb{k}),
$$

where the distinct irreducible representations $V_{1}, \ldots, V_{m}$ are each realized as an irreducible representation of one of the matrix algebras $M_{n_{i}}(\mathbb{k})$. Let $f_{1}, \ldots, f_{m}$ denote the distinct pairwise orthogonal central idempotents of $\mathbb{k}[\Gamma]$; each $f_{i}$ is the identity matrix in the matrix algebra $M_{n_{i}}(\mathbb{k})$, and

$$
1=\sum_{i} f_{i} \in \mathbb{k}[\Gamma]
$$

The above is not, in general, a minimal decomposition of 1 as a sum of orthogonal idempotents, since the idempotents $f_{i}$ themselves are not minimal if $\operatorname{dim}\left(V_{i}\right)>1$. For each $i$ and $s=1, \ldots, n_{i}$, let $e_{i, s}$ denote the matrix unit of $M_{n_{i}}(\mathbb{k})$ whose $(s, s)$ entry is equal to one and whose other entries are zero. Then the $e_{i, s}$ are minimal orthogonal idempotents in $\mathbb{k}[\Gamma]$, with

$$
f_{i}=\sum_{s=1}^{n_{i}} e_{i, s} .
$$

An important role will be played by the (super)algebra

$$
B_{n}^{\Gamma}:=\left(B^{\Gamma} \otimes \cdots \otimes B^{\Gamma}\right) \rtimes S_{n} .
$$

The above tensor product is understood as the tensor product in the category of superalgebras, and the action of $S_{n}$ is by superpermutations: if $s_{k} \in S_{n}$ is the simple transposition $(k, k+1)$, then

$s_{k} \cdot\left(b_{1} \otimes \cdots \otimes b_{k} \otimes b_{k+1} \otimes \cdots \otimes b_{n}\right)=(-1)^{\left|b_{k}\right|\left|b_{k+1}\right|} b_{1} \otimes \cdots \otimes b_{k+1} \otimes b_{k} \otimes \cdots \otimes b_{n}$.

The degree 0 subalgebra $\mathbb{k}\left[\Gamma^{n} \rtimes S_{n}\right] \subset B_{n}^{\Gamma}$ contains all the idempotents of $B_{n}^{\Gamma}$. This subalgebra is isomorphic to a direct product of matrix algebras

$$
\mathbb{k}\left[\Gamma^{n} \rtimes S_{n}\right]=M_{l_{1}}(\mathbb{k}) \times \cdots \times M_{l_{s}}(\mathbb{k})
$$

with one matrix algebra for each isomorphism class of irreducible $\mathbb{k}\left[\Gamma^{n} \rtimes S_{n}\right]$ modules. Just as the isomorphism classes of irreducible $\mathbb{k}\left[S_{n}\right]$-modules are parameterized by partitions of $n$, the isomorphism classes of irreducible $\mathbb{k}\left[\Gamma^{n} \rtimes S_{n}\right]$-modules 
are parameterized by partition-valued functions of $I_{\Gamma}$ (i.e., $\left|I_{\Gamma}\right|$-tuples of partitions of $n$ ).

\subsection{The category $\mathscr{H}_{\Gamma}^{\prime}$}

We define an additive, $\mathbb{k}$-linear, $\mathbb{Z}$-graded 2-category $\mathscr{H}_{\Gamma}^{\prime}$ as follows. The objects of $\mathscr{H}_{\Gamma}^{\prime}$ are indexed by the integers $\mathbb{Z}$. The 1 -morphisms are generated by $P$ and $Q$, where for each $n, P$ denotes a 1-morphism from $n$ to $n+1$ and $Q$ is a 1-morphism from $n+1$ to $n$. Thus a 1 -morphism of $\mathscr{H}_{\Gamma}^{\prime}$ is a finite composition (sequence) of $P$ 's and $Q$ 's. The identity 1-morphism of $n$ is denoted by $\mathbf{1}$ (the empty sequence).

\section{Remark 3}

Technically, we should write $P(n)$ and $(n) Q$ to identify the domain and range of $P$ and $Q$. However, the properties of $P$ and $Q$ do not depend on $n$, so we usually omit this extra parameter in order to simplify notation. We could have chosen to define $\mathscr{H}_{\Gamma}^{\prime}$ as a monoidal 1-category instead of as a 2-category, but we prefer to consider $\mathscr{H}_{\Gamma}^{\prime}$ as a 2-category because this better parallels the story for Kac-Moody Lie algebras in [15], [16], [17], and [24].

The space of 2-morphisms between two 1-morphisms is a $\mathbb{Z}$-graded $\mathbb{k}$-algebra generated by suitable planar diagrams modulo local relations. The diagrams consist of oriented compact 1 -manifolds immersed into the plane strip $\mathbb{R} \times[0,1]$ modulo isotopies fixing the boundary which preserve the relative height of dots. (Isotopic diagrams which change the relative height of dots are equal to one another up to a sign, as will be indicated below.) The source of a given 2-morphism lies on the boundary $y=0$, while the target lies on $y=1$. Thus 2-morphisms are read from bottom to top.

A single upward-oriented strand denotes the identity 2-morphism id $: P \rightarrow P$, while a downward-oriented strand denotes the identity 2-morphism id : $Q \rightarrow Q$ :

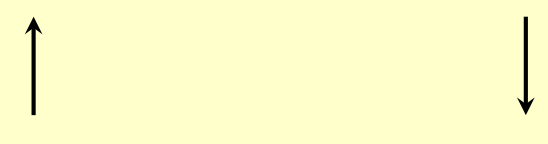

An upward-oriented line is allowed to carry dots labeled by elements $b \in B^{\Gamma}$. For example,

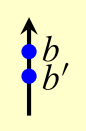

is an element of $\operatorname{Hom}_{\mathcal{H}_{\Gamma}^{\prime}}(P, P)$, while 


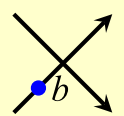

is an element of $\operatorname{Hom}_{\mathscr{H}_{\Gamma}^{\prime}}(P Q, Q P)$. Note that the domain of a 2-morphism is specified at the bottom of the diagram and the codomain is specified at the top, and compositions of 2-morphisms are read from bottom to top.

The local relations we impose are the following. First we have relations involving the movement of dots along the carrier strand. We allow dots to move freely along strands and through intersections:
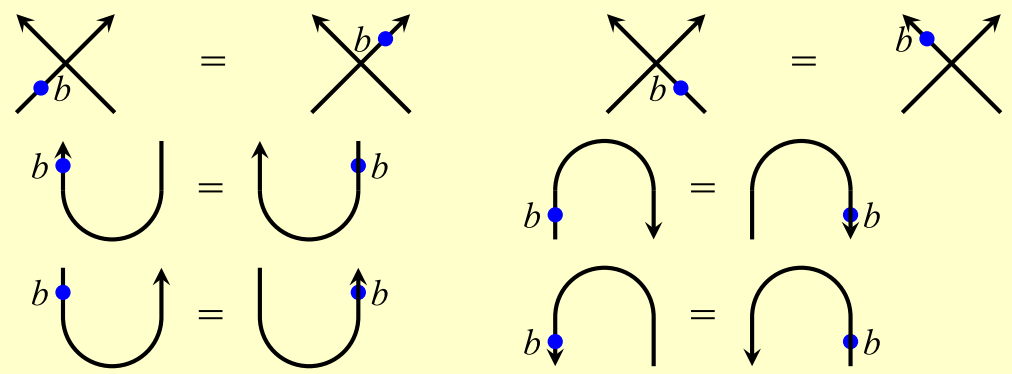

The U-turn 2-morphisms are adjunctions making $P$ and $Q$ biadjoint up to a grading shift that will be defined later in this section. Collision of dots is controlled by the algebra structure on $B^{\Gamma}$ :
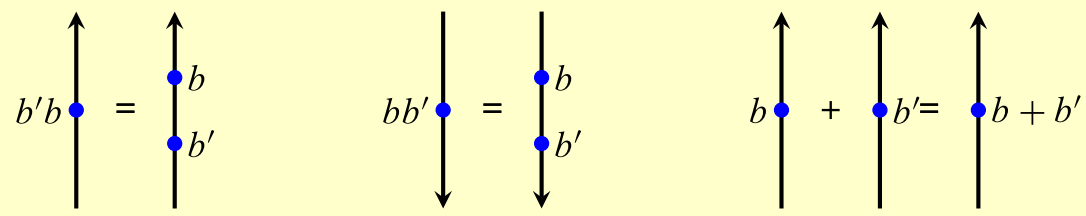

Note that dots collide, so to speak, in the direction of the arrow.

Dots on distinct strands supercommute when they move past one another:

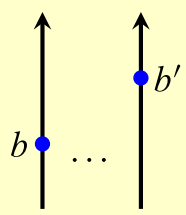

$$
=(-1)^{|b|\left|b^{\prime}\right|} b \uparrow_{\ldots}^{b \oint^{\prime}}
$$

In addition to specifying how dots collide and slide we impose the following local relations in the $\Gamma$ graphical calculus: 

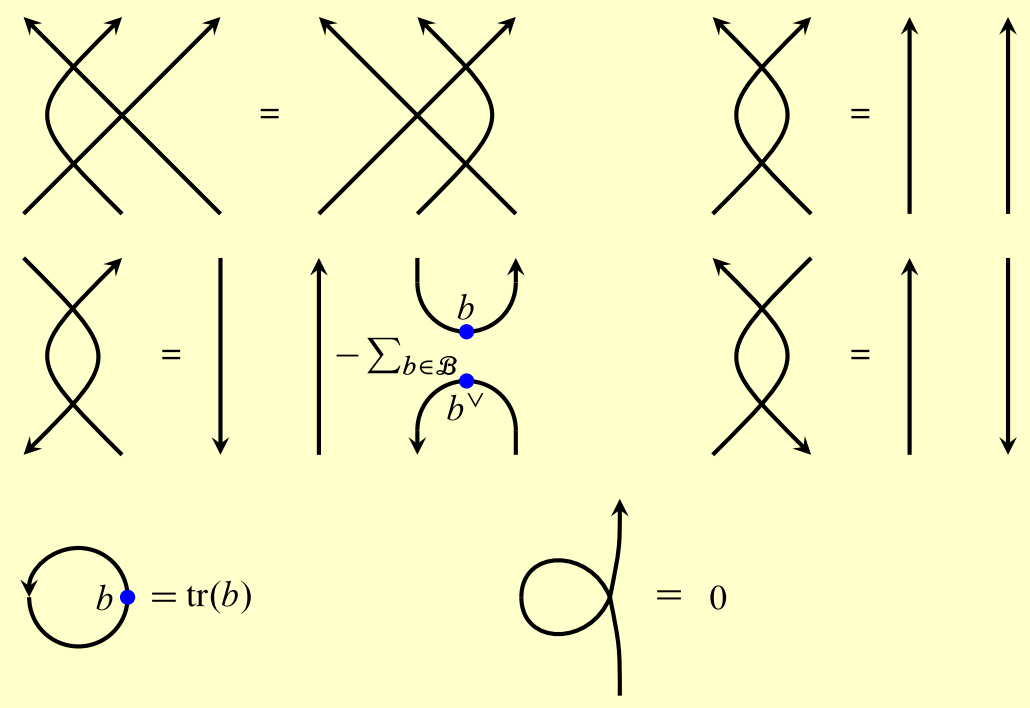

In the first equation on line (10) above, the summation is taken over a basis $\mathscr{B}$ of $B^{\Gamma}$ - this morphism is easily seen to be independent of the choice of basis. We assign a $\mathbb{Z}$-grading to the space of planar diagrams by defining
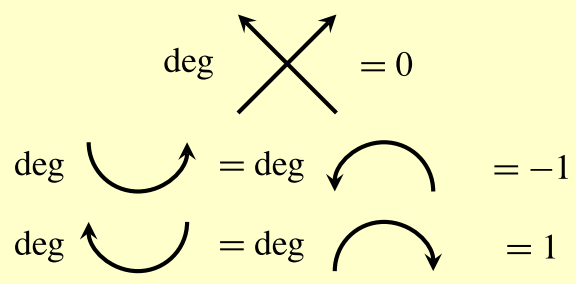

and by defining the degree of a dot labeled by $b$ to be the degree of $b$ in the graded algebra $B^{\Gamma}$. When equipped with these assignments all of the graphical relations are graded. Thus $\operatorname{Hom}_{\mathscr{H}_{\Gamma}^{\prime}}(\cdot, \cdot)$ is a $\mathbb{Z}$-graded vector space and composition of morphisms is compatible with the grading.

Because of the relation dictating how dots slide through crossings, there is a natural map from the semidirect product

$$
B_{n}^{\Gamma}:=\left(B^{\Gamma} \otimes \cdots \otimes B^{\Gamma}\right) \rtimes S_{n}
$$

to the endomorphism algebra $\operatorname{Hom}_{\mathscr{H}_{\Gamma}^{\prime}}\left(P^{n}, P^{n}\right)$ whose image is the subalgebra spanned by braid-like diagrams (i.e., diagrams with no local maxima or local minima). Notice that if we denote by $T_{k}$ the upward crossings of the $k$ and $(k+1)$ st adjacent strands and by $X_{k}(b)$ the dots labeled $b \in B^{\Gamma}$ on the $k$ th strand, then this subalgebra is generated by $T_{1}, \ldots, T_{n-1}$ and $X_{1}(b), \ldots, X_{n}(b)$ for $b \in B^{\Gamma}$. The $T$ 's and $X$ 's are subject to the following relations: 


$$
\begin{aligned}
T_{k}^{2} & =1 \quad \text { and } \quad T_{k} T_{l}=T_{l} T_{k} \quad \text { for }|k-l|>1, \\
T_{k} T_{k+1} T_{k} & =T_{k+1} T_{k} T_{k+1} \quad \text { for all } k=1, \ldots, n-2, \\
T_{k} X_{k}(b) & =X_{k+1}(b) T_{k} \quad \text { for all } k=1, \ldots, n-1, \\
X_{k}(b) X_{k}\left(b^{\prime}\right) & =X_{k}\left(b b^{\prime}\right) \quad \text { and } \\
X_{k}(b) X_{l}\left(b^{\prime}\right) & =(-1)^{|b|\left|b^{\prime}\right|} X_{l}\left(b^{\prime}\right) X_{k}(b) \quad \text { if } k \neq l .
\end{aligned}
$$

We will write $T$ and $X(b)$ instead of $T_{k}$ and $X_{k}(b)$ when the subscript is understood. In fact, it will follow from the construction of Section 9 that the map $B_{n}^{\Gamma} \rightarrow$ $\operatorname{End}_{\mathscr{H}_{\Gamma}^{\prime}}\left(P^{n}\right)$ is injective, so that the subalgebra of endomorphisms spanned by braidlike diagrams is isomorphic $B_{n}^{\Gamma}$ (see Remark 8).

\subsection{The category $\mathscr{H}_{\Gamma}$}

We define $\mathscr{H}_{\Gamma}$ to be the Karoubi envelope (also known as the idempotent completion) of $\mathscr{H}_{\Gamma}^{\prime}$. By definition, the objects of $\mathscr{H}_{\Gamma}$ are also indexed by $\mathbb{Z}$, while a 1 -morphism consists of a pair $(R, e)$, where $R$ is a 1-morphism of $\mathscr{H}_{\Gamma}^{\prime}$ and $e: R \rightarrow R$ is an idempotent endomorphism $e^{2}=e$. Morphisms from $(R, e)$ to $\left(R^{\prime}, e^{\prime}\right)$ are morphisms $g: R \rightarrow R^{\prime}$ such that $g e=g$ and $e^{\prime} g=g$. The idempotent $e$ defines the identity morphism of $(R, e)$.

Since $\mathscr{H}_{\Gamma}$ is a graded 2-category, the (split) Grothendieck group $K_{0}\left(\mathscr{H}_{\Gamma}\right)$ of $\mathscr{H}_{\Gamma}$ is a $\mathbb{k}\left[q, q^{-1}\right]$-linear category where multiplication by $q$ corresponds to the shift $\langle 1\rangle$. (We will assume Grothendieck groups are tensored with the base field $\mathbb{k}$.) The objects of $K_{0}\left(\mathscr{H}_{\Gamma}\right)$ are the same as the objects of $\mathscr{H}_{\Gamma}$, namely the integers. The space of morphisms $\operatorname{Hom}_{K_{0}\left(\mathscr{H}_{\Gamma}\right)}(n, m)$ is the split Grothendieck group of the additive category $\operatorname{Hom}_{\mathscr{H}_{\Gamma}}(n, m)$. Composition of 1-morphisms in $\mathscr{H}_{\Gamma}$ gives the Grothendieck group $K_{0}\left(\mathscr{H}_{\Gamma}\right)$ the structure of a $\mathbb{k}$-algebra.

\subsection{First main theorem}

\section{THEOREM 1}

There is a canonical isomorphism of algebras:

$$
\pi: \mathfrak{h}_{\Gamma} \stackrel{\sim}{\rightarrow} K_{0}\left(\mathcal{H}_{\Gamma}\right) .
$$

This theorem will be proved in Section 7. In the rest of this section we want to sketch how the morphism $\pi$ comes about.

To do this consider various idempotent 2-morphisms in the category $\mathscr{H}_{\Gamma}^{\prime}$. Since $M_{n_{i}}(\mathbb{k}) \subset \mathbb{k}[\Gamma] \subset B^{\Gamma} \subset \operatorname{End}_{\mathcal{H}_{\Gamma}^{\prime}}(P)$, any idempotent $e \in M_{n_{i}}(\mathbb{k})$ gives rise (for any $n \in \mathbb{Z})$ to a 1 -morphism $(P, e)$ in $\operatorname{Hom}_{\mathscr{H}_{\Gamma}}(n, n+1)$. Let $\tilde{P}_{i}=\left(P, f_{i}\right) \in \mathscr{H}_{\Gamma}$, where 
the $f_{i}$ are the pairwise orthogonal central idempotents of $\mathbb{C}[\Gamma]$ described in Section 3.1.1.

LEMMA 2

There is an isomorphism of 1-morphisms in the category $\mathscr{H}_{\Gamma}$ :

$$
P \cong \bigoplus_{i=1}^{m} \tilde{P}_{i} .
$$

\section{Proof}

The idempotents $f_{i}$ define a 2 -morphism from $\bigoplus_{i=1}^{m} \tilde{P}_{i}$ to $P$ in $\mathscr{H}_{\Gamma}$. Since $1=$ $\sum_{i=1}^{m} f_{i}$ and $f_{i} f_{j}=\delta_{i, j} f_{i}$, this 2-morphism is an isomorphism.

The 1-morphisms $\tilde{P}_{i}$ can be further decomposed in $\mathscr{H}_{\Gamma}$ whenever the idempotents $f_{i}$ are not minimal. Recall the minimal idempotents $e_{i, 1}, \ldots, e_{i, n_{i}}$ from Section 3.1.1. Note that for fixed $i$ the $e_{i, j}$, which are distinct idempotents in $M_{n_{i}}(\mathbb{k})$, define isomorphic representations $\mathbb{C}[\Gamma] e_{i, 1} \cong \mathbb{C}[\Gamma] e_{i, l}$ of $\mathbb{C}[\Gamma]$. In particular, the objects $\left(P, e_{i, j}\right)$ for distinct $j$ are all isomorphic in $\mathscr{H}_{\Gamma}$. Let $P_{i}$ denote the 1morphism $\left(P, e_{i, 1}\right) \in \mathscr{H}_{\Gamma}$. Then we have the following.

\section{LEMMA 3}

There is an isomorphism in $\mathscr{H}_{\Gamma}$,

$$
\tilde{P}_{i} \cong \bigoplus_{j=1}^{n_{i}} P_{i}^{\oplus n_{i}},
$$

where $n_{i}$ is the dimension (as a $\mathbb{k}$ vector space) of the $\mathbb{k}[\Gamma]$-module $V_{i}$.

\section{Proof}

The proof is just as in Lemma 2, using the fact that $f_{i}=\sum_{j=1}^{n_{i}} e_{i, j}$.

We will prove later in Section 7 that the objects $P_{i} \in \mathscr{H}_{\Gamma}$ are indecomposable.

The canonical adjunctions in $\mathscr{H}_{\Gamma}^{\prime}$ involving $P$ and $Q$ (the cups and caps) also allow us to decompose $Q$, since any idempotent $e \in \operatorname{End}_{\mathscr{H}_{\Gamma}^{\prime}}(P)$ gives rise to an idempotent $e \in \operatorname{End}_{\mathscr{H}_{\Gamma}^{\prime}}(Q)$. We define 1-morphisms $Q_{i} \in \mathscr{H}_{\Gamma}$ by $Q_{i}=\left(Q, e_{i, 1}\right)$. It follows immediately by adjunction that $Q_{i}$ is indecomposable too.

A basic consequence of the relations between 2-morphisms in $\mathscr{H}_{\Gamma}$ is the following result proved in Section 6. 
PROPOSITION 1

For $i, j \in I_{\Gamma}$, we have

$$
Q_{j} P_{i} \cong \begin{cases}P_{i} Q_{j} \oplus \mathrm{id}\langle-1\rangle \oplus \mathrm{id}\langle 1\rangle & \text { if } i=j, \\ P_{i} Q_{j} \oplus \mathrm{id} & \text { if }\langle i, j\rangle=-1,\end{cases}
$$

where $\langle\cdot\rangle$ denotes the grading shift in $\mathscr{H}_{\Gamma}$.

More generally, we define 1-morphisms $P_{i}^{(n)}$ and $Q_{i}^{(n)}$ by

$$
P_{i}^{(n)}=\left(P^{n}, e_{\text {triv }, i, 1}\right) \quad \text { and } \quad Q_{i}^{(n)}=\left(Q^{n}, e_{\text {triv }, i, 1}\right),
$$

where the idempotent $e_{\text {triv }, i, 1}$ is a minimal idempotent in $M_{n_{i}}(\mathbb{k})^{n} \rtimes S_{n} \subset \mathbb{k}\left[\Gamma^{n} \rtimes S_{n}\right]$ corresponding to the trivial representation of $S_{n}$. In Section 6 we prove the following proposition.

\section{PROPOSITION 2}

For $i, j \in I_{\Gamma}$, we have isomorphisms in $\mathscr{H}_{\Gamma}$,

$$
\begin{aligned}
P_{i}^{(n)} P_{j}^{(m)} \cong P_{j}^{(m)} P_{i}^{(n)} \quad \text { and } \quad Q_{i}^{(n)} Q_{j}^{(m)} \cong Q_{j}^{(m)} Q_{i}^{(n)} \text { for all } i, j \in I_{\Gamma}, \\
Q_{i}^{(n)} P_{j}^{(m)} \cong \begin{cases}\bigoplus_{k \geq 0} P_{i}^{(m-k)} Q_{i}^{(n-k)} \otimes H^{\star}\left(\mathbb{P}^{k}\right) & \text { if } i=j \in I_{\Gamma}, \\
P_{j}^{(m)} Q_{i}^{(n)} \oplus P_{j}^{(m-1)} Q_{i}^{(n-1)} & \text { if }\langle i, j\rangle=-1, \\
P_{j}^{(m)} Q_{i}^{(n)} & \text { if }\langle i, j\rangle=0,\end{cases}
\end{aligned}
$$

where $H^{\star}\left(\mathbb{P}^{k}\right)$ denotes the graded cohomology of projective spaces $\mathbb{P}^{k}$ shifted so that it lies in degrees $-k,-k+2, \ldots, k-2, k$.

Notice that in the third equation above the graded dimension of $H^{\star}\left(\mathbb{P}^{k}\right)$ is the quantum integer $[k+1]$. By convention, $H^{\star}\left(\mathbb{P}^{k}\right)=0$ if $k<0$. Also, $P_{i}^{(l)}=Q_{i}^{(l)}=0$ when $l<0$, so the summand corresponding to a $k>\min (n, m)$ is zero (so the direct sum on the right is finite).

It follows immediately from Proposition 2 that there is a well-defined morphism

$$
\pi: \mathfrak{h}_{\Gamma} \longrightarrow K_{0}\left(\mathcal{H}_{\Gamma}\right)
$$

given by sending $p_{i}^{(n)}$ to the class $\left[P_{i}^{(n)}\right]$ and sending $q_{j}^{(m)}$ to the class $\left[Q_{j}^{(m)}\right]$. That the map $\pi$ is an isomorphism is a rigorous formulation of the informal statement " $\mathscr{H}_{\Gamma}$ categorifies the Heisenberg algebra."

\subsection{Remarks on relations in $\mathscr{H}_{\Gamma}^{\prime}$}

We end this section with some remarks on the relations from lines (9)-(11). The second relation in (11) is natural for degree reasons; in fact, imposing this relation is 
equivalent to declaring that the object $P$ does not have negative-degree endomorphisms.

Moreover, if we believe that the identity should not have negative-degree endomorphisms, then the left relation in (11) is immediate unless $a$ has degree 2; that is, unless $a$ is a linear combination of elements of the form $\left(v_{1} \wedge v_{2}, \gamma\right)$. Since

$$
\left(v_{1} \wedge v_{2}, \gamma\right)=\left(v_{1}, \gamma\right) \cdot\left(\gamma^{-1} v_{2}, 1\right)
$$

from moving $\gamma^{-1} v_{2}$ around the circle we get

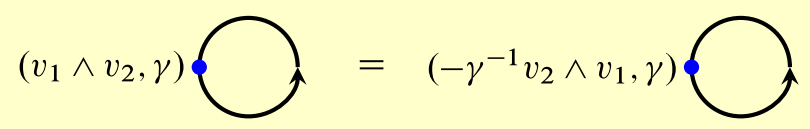

where the minus sign is a consequence of passing the dot labeled $\left(v_{1}, \gamma\right)$ by the dot labeled $\left(\gamma^{-1} v_{2}, 1\right)$. Since $\gamma$ acts nontrivially on $V$, it follows that the counterclockwise circle with a solid dot labeled by $\left(v_{1} \wedge v_{2}, \gamma\right)$ is zero unless $\gamma=1$.

To understand the relation imposed when $\gamma=1$, we add a cup at the bottom of the first relation in (10). Simplifying, we obtain

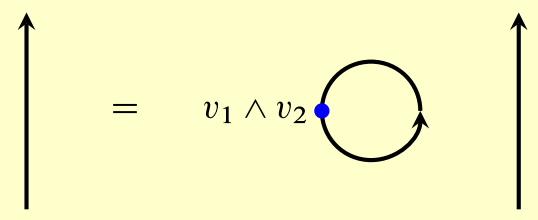

It follows that if the closed diagram which appears above is a scalar, then it must be equal to one. Alternatively, if we multiply the upward strand by $v_{1} \wedge v_{2}$ and close off we get that the counterclockwise circle with a solid dot labeled by $v_{1} \wedge v_{2}$ is an idempotent. So, if this idempotent were not equal to one, then the identity map would break up into a direct sum of multiple 1-morphisms in the idempotent completion. This would result in extra unwanted 1-morphisms in our Heisenberg category $\mathscr{H}_{\Gamma}$.

\subsubsection{Further relations}

Although left-twist curls on an upward-pointing strand are zero, right-twist curls (which have degree 2) may be nonzero. For shorthand, we will draw right-twist curls as hollow dots:

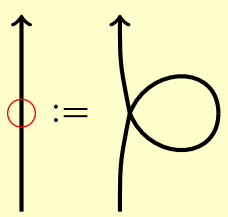


To pass a solid dot past a hollow dot involves sliding the solid dot along the right-twist curl. Thus solid dots commute with hollow dots:

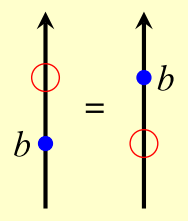

Hollow dots have an interesting "affine Hecke"-type relation with crossings, which involves the creation of labeled solid dots,

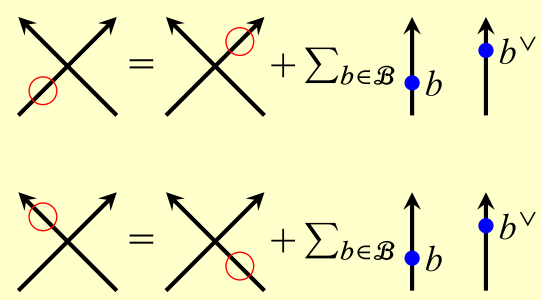

where again the summations are over the basis $\mathscr{B}$. These relations are reminiscent of relations in the degenerate affine Hecke algebra associated to the symmetric group and its wreath products (see, e.g., [23], [27], [28]). The proof of these relations is an easy calculation using the defining relations for 2-morphisms in $\mathscr{H}_{\Gamma}^{\prime}$.

Finally, it also follows directly from the graphical relations that the triple point move,

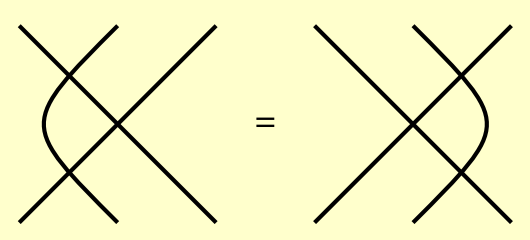

which was defined to hold when all three strands are oriented up, in fact holds in all possible orientations.

\section{A 2-representation of $\mathscr{H}_{\Gamma}$}

In this section we construct an action of $\mathscr{H}_{\Gamma}$ on the derived categories of finitedimensional graded modules over certain algebras $A_{n}^{\Gamma}$. This induces an action on derived categories of coherent sheaves on Hilbert schemes and allows us to show in the next section that our categories $\mathscr{H}_{\Gamma}$ are nondegenerate (subsequently proving Theorem 1). 


\subsection{Categories}

Since $\Gamma \subset \mathrm{SL}_{2}(\mathbb{k})$ acts on $V \cong \mathbb{k}^{2}$ it acts naturally on $\operatorname{Sym}^{*}\left(V^{\vee}\right)$, and we denote by $\operatorname{Sym}^{*}\left(V^{\vee}\right) \rtimes \Gamma$ their semidirect product (also known as the smash product $\left.\operatorname{Sym}^{*}\left(V^{\vee}\right) \# \mathbb{k}[\Gamma]\right)$. For instance, if $\Gamma=\mathbb{Z} / n \mathbb{Z}$, identified as $n$th roots of unity, then we can identify $\operatorname{Sym}^{*}\left(V^{\vee}\right)$ with $\mathbb{k}[x, y]$ and the action is $\zeta \cdot(x, y)=\left(\zeta x, \zeta^{-1} y\right)$. $\operatorname{Sym}^{*}\left(V^{\vee}\right) \rtimes \Gamma$ is a $\mathbb{k}$-algebra, and an element of $\operatorname{Sym}^{*}\left(V^{\vee}\right) \rtimes \Gamma$ is a linear combination of elements of the form $(f, \gamma)$, where $f \in \operatorname{Sym}^{*}\left(V^{\vee}\right)$ and $\gamma \in \Gamma$ with multiplication given by

$$
(f, \gamma) \cdot\left(f^{\prime}, \gamma^{\prime}\right)=\left(f \gamma \cdot f^{\prime}, \gamma \gamma^{\prime}\right)
$$

For each nonnegative integer $n$ define

$$
A_{n}^{\Gamma}:=\left[\left(\operatorname{Sym}^{*}\left(V^{\vee}\right) \rtimes \Gamma\right) \otimes\left(\operatorname{Sym}^{*}\left(V^{\vee}\right) \rtimes \Gamma\right) \otimes \cdots \otimes\left(\operatorname{Sym}^{*}\left(V^{\vee}\right) \rtimes \Gamma\right)\right] \rtimes S_{n},
$$

where $S_{n}$ is the symmetric group acting by permuting the $n$ terms in the product. Notice that here we use the ordinary tensor product and not the super tensor product used to define $B_{n}^{\Gamma}$. These algebras inherit the natural grading from $\operatorname{Sym}^{*}\left(V^{\vee}\right)$. We denote by $D\left(A_{n}^{\Gamma}\right.$-gmod $)$ the bounded derived category of finite-dimensional, graded (left) $A_{n}^{\Gamma}$-modules.

We have maps,

$$
\mathbb{k}[\Gamma] \stackrel{i}{\rightarrow} A_{1}^{\Gamma} \stackrel{p}{\rightarrow} \mathbb{k}[\Gamma],
$$

where the first denotes the natural inclusion of the group algebra $\mathbb{k}[\Gamma]$ and the second is the projection which takes $\operatorname{Sym}^{>0}(V)$ to zero. Thus any $\mathbb{k}[\Gamma]$-module is also an $A_{1}^{\Gamma}$-module and vice versa.

\subsection{Functors $\mathrm{P}$ and $\mathrm{Q}$}

Consider the natural inclusion $A_{n}^{\Gamma} \otimes A_{1}^{\Gamma} \rightarrow A_{n+1}^{\Gamma}$ making use of the embedding $S_{n}=$ $S_{n} \times S_{1} \hookrightarrow S_{n+1}$. This gives $A_{n+1}^{\Gamma}$ and $A_{n}^{\Gamma} \otimes \mathbb{k}[\Gamma]$ the structure of a module over $A_{n}^{\Gamma} \otimes A_{1}^{\Gamma}$.

Define the $\left(A_{n+1}^{\Gamma}, A_{n}^{\Gamma}\right)$-bimodule

$$
P^{\Gamma}(n):=A_{n+1}^{\Gamma} \otimes_{A_{n}^{\Gamma} \otimes A_{1}^{\Gamma}}\left(A_{n}^{\Gamma} \otimes \mathbb{k}[\Gamma]\right)
$$

and the $\left(A_{n}^{\Gamma}, A_{n+1}^{\Gamma}\right)$-bimodule

$$
\text { (n) } Q^{\Gamma}:=\left(A_{n}^{\Gamma} \otimes \mathbb{k}[\Gamma]\right) \otimes_{A_{n}^{\Gamma} \otimes A_{1}^{\Gamma}} A_{n+1}^{\Gamma} .
$$

Define the functor $\mathrm{P}(n): D\left(A_{n}^{\Gamma}-\mathrm{gmod}\right) \rightarrow D\left(A_{n+1}^{\Gamma}-\mathrm{gmod}\right)$ by

$$
\mathrm{P}(n)(\cdot):=P^{\Gamma}(n) \otimes_{A_{n}^{\Gamma}}(\cdot) .
$$


Similarly, we define $(n) \mathrm{Q}: D\left(A_{n+1}^{\Gamma}-\right.$ gmod $) \rightarrow D\left(A_{n}^{\Gamma}-\right.$ gmod $)$ by

$$
(n) \mathrm{Q}(\cdot):=(n) Q^{\Gamma} \otimes_{A_{n+1}^{\Gamma}}(\cdot)[-1]\{1\},
$$

where $[\cdot]$ denotes the cohomological shift, while $\{\cdot\}$ denotes the grading shift. The relationship between these shifts and the shift $\langle\cdot\rangle$ in $\mathscr{H}_{\Gamma}$ is that $\langle 1\rangle=[1]\{-1\}$, as we will see. We will also usually omit the $(n)$ from the notation for functors and just write $\mathrm{P}$ or $\mathrm{Q}$.

\subsection{Natural transformations}

In order to define an $\mathscr{H}_{\Gamma}$-action on $\bigoplus_{n} D\left(A_{n}^{\Gamma}\right.$-gmod $)$ we also need to define the following natural transformations:

(1) $\quad X(v): \mathrm{P} \rightarrow \mathrm{P}[1]\{-1\}$ and $X(v): \mathrm{Q} \rightarrow \mathrm{Q}[1]\{-1\}$ for any $v \in V$;

(2) $\quad X(\gamma): \mathrm{P} \rightarrow \mathrm{P}$ and $X(\gamma): \mathrm{Q} \rightarrow \mathrm{Q}$ for any $\gamma \in \Gamma$;

(3) $T: \mathrm{PP} \rightarrow \mathrm{PP}, T: \mathrm{QQ} \rightarrow \mathrm{QQ}, T: \mathrm{QP} \rightarrow \mathrm{PQ}$ and $T: \mathrm{PQ} \rightarrow \mathrm{QP}$;

(4) $\operatorname{adj}: \mathrm{QP} \rightarrow \mathrm{id}[-1]\{1\}$ and $\operatorname{adj}: \mathrm{PQ} \rightarrow \mathrm{id}[1]\{-1\}$; and

(5) $\quad \operatorname{adj}:$ id $\rightarrow$ QP $[-1]\{1\}$ and adj: id $\rightarrow$ PQ $[1]\{-1\}$.

These natural transformations define the action of the following 2-morphisms in $\mathscr{H}_{\Gamma}^{\prime}$ (as usual we read the diagrams from the bottom up):
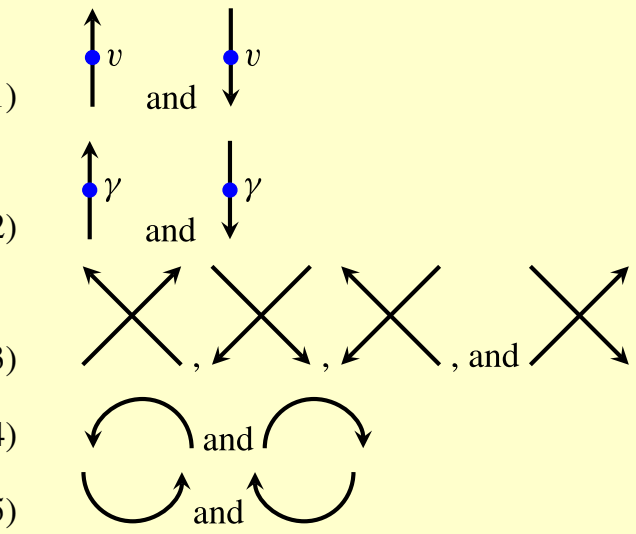

\subsubsection{Preliminaries}

Since we will deal with tensor products of complexes, we briefly review some standard conventions. Given two complexes

$$
A_{\bullet}=\cdots \rightarrow A_{i} \stackrel{d}{\rightarrow} A_{i+1} \rightarrow \cdots \quad \text { and } \quad B_{\bullet}=\cdots \rightarrow B_{i} \stackrel{d}{\rightarrow} B_{i+1} \rightarrow \cdots,
$$

the tensor product $A_{\bullet} \otimes B_{\bullet}$ is the complex with terms $\bigoplus_{i+j=k} A_{i} \otimes B_{j}$ and differential

$A_{i} \otimes B_{j} \stackrel{d \otimes 1+(-1)^{i} 1 \otimes d}{\longrightarrow} A_{i+1} \otimes B_{j} \oplus A_{i} \otimes B_{j+1}$ 
Given a map $f: A_{\bullet} \rightarrow A_{\bullet}[k]$ of complexes induced by $f_{i}: A_{i} \rightarrow A_{i+k}$ we get a map $f \otimes 1: A_{\bullet} \otimes B_{\bullet} \rightarrow A_{\bullet} \otimes B_{\bullet}[k]$ induced by

$$
(f \otimes 1)_{i}=f_{i} \otimes 1: A_{i} \otimes B_{j} \rightarrow A_{i+k} \otimes B_{j} .
$$

Similarly, given a map $g: B_{\bullet} \rightarrow B_{\bullet}[k]$ induced by $g_{i}: B_{i} \rightarrow B_{i+k}$ we get a map $1 \otimes g$ induced by

$$
(1 \otimes g)_{i}=(-1)^{i k} g_{i} \otimes 1: A_{i} \otimes B_{j} \rightarrow A_{i} \otimes B_{j+k} .
$$

Notice that if $k=1$, then $(f \otimes 1)$ and $(1 \otimes g)$ anticommute.

\subsubsection{Definition of $X \in \operatorname{End}(P)$}

We define a map $X(\gamma): \mathbb{k}[\Gamma] \rightarrow \mathbb{k}[\Gamma]$ of left $A_{1}^{\Gamma}$-modules via multiplication on the right $\gamma^{\prime} \mapsto \gamma^{\prime} \cdot \gamma$. This induces a map $X(\gamma): P^{\Gamma}(n) \rightarrow P^{\Gamma}(n)$ of $\left(A_{n+1}^{\Gamma}, A_{n}^{\Gamma}\right)$ bimodules.

To define $X(v)$ we have to work harder. Consider $A_{1}^{\Gamma} \otimes V^{\vee}$, where the tensor product is over $\mathbb{k}$ (so $A_{1}^{\Gamma}$ only acts on the left factor by multiplication on the left). Then the multiplication map $\operatorname{Sym}^{*}\left(V^{\vee}\right) \otimes V^{\vee} \rightarrow \operatorname{Sym}^{*}\left(V^{\vee}\right)$ induces a map $d: A_{1}^{\Gamma} \otimes$ $V^{\vee} \rightarrow A_{1}^{\Gamma}$ of left $A_{1}^{\Gamma}$-modules via

$$
(f, \gamma) \otimes w \mapsto(f \gamma \cdot w, \gamma),
$$

where $f \in \operatorname{Sym}^{*}(V), \gamma \in \Gamma$, and $w \in V^{\vee}$. Similarly, we have a map $d: A_{1}^{\Gamma} \otimes$ $\bigwedge^{2} V^{\vee} \rightarrow A_{1}^{\Gamma} \otimes V^{\vee}$ given by

$$
(f, \gamma) \otimes\left(w_{1} \wedge w_{2}\right) \mapsto\left(f\left(\gamma w_{1}\right), \gamma\right) \otimes w_{2}-\left(f\left(\gamma w_{2}\right), \gamma\right) \otimes w_{1} .
$$

Using these maps we can define the following free resolution of the left $A_{1}^{\Gamma}$ module $\mathbb{k}[\Gamma]$ :

$$
0 \rightarrow A_{1}^{\Gamma} \otimes \bigwedge^{2} V^{\vee} \stackrel{d}{\rightarrow} A_{1}^{\Gamma} \otimes V^{\vee} \stackrel{d}{\rightarrow} A_{1}^{\Gamma} \rightarrow \mathbb{k}[\Gamma] .
$$

Suppose that $v \in V$. We need to define a map $X(v): \mathbb{k}[\Gamma] \rightarrow \mathbb{k}[\Gamma][1]\{-1\}$ inside $D\left(A_{1}^{\Gamma}-\right.$ gmod $)$. To do this, define

$$
\phi_{v}: A_{1}^{\Gamma} \otimes V^{\vee} \rightarrow A_{1}^{\Gamma}\{-1\}
$$

to be the map induced by $(f, \gamma) \otimes w \mapsto\langle w, v\rangle(f, \gamma)$, where $\langle\cdot, \cdot\rangle: V^{\vee} \otimes V \rightarrow \mathbb{k}$ is the natural pairing. It is easy to check that this map is a map of graded left $A_{1}^{\Gamma}$-modules.

Similarly, define

$$
\phi_{v}^{\prime}: A_{1}^{\Gamma} \otimes \bigwedge^{2} V^{\vee} \rightarrow A_{1}^{\Gamma} \otimes V^{\vee}\{-1\}
$$


to be the map induced by

$$
(f, \gamma) \otimes\left(w_{1} \wedge w_{2}\right) \mapsto\left\langle w_{1}, v\right\rangle(f, \gamma) \otimes w_{2}-\left\langle w_{2}, v\right\rangle(f, \gamma) \otimes w_{1} .
$$

Using these maps, we can write down the following commutative diagram:

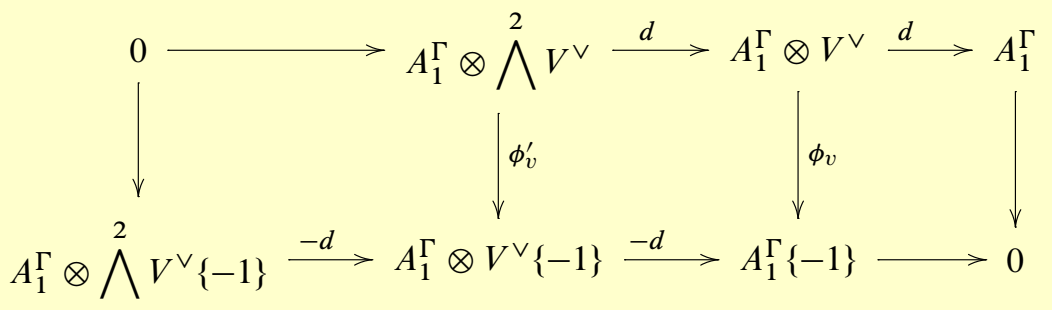

The commutativity of the middle square is an easy exercise. (Note that the differential is $-d$ in the second row since shifting the complex by one negates the differential.)

The map from (13) induces a map $X(v): \mathbb{k}[\Gamma] \rightarrow \mathbb{k}[\Gamma][1]\{-1\}$ and subsequently a map $X(v): P^{\Gamma}(n) \rightarrow P^{\Gamma}(n)[1]\{-1\}$ (in the derived category) of graded $\left(A_{n+1}^{\Gamma}, A_{n}^{\Gamma}\right)$ bimodules.

\subsubsection{Definition of $X \in \operatorname{End}(Q)$}

If $\gamma \in \Gamma$, then multiplication on the left induces a map $X(\gamma): \mathbb{k}[\Gamma] \rightarrow \mathbb{k}[\Gamma]$ of right $A_{1}^{\Gamma}$-modules via $\gamma^{\prime} \mapsto \gamma \cdot \gamma^{\prime}$ and subsequently a map $X(\gamma):(n) Q^{\Gamma} \rightarrow(n) Q^{\Gamma}$ of $\left(A_{n}^{\Gamma}, A_{n+1}^{\Gamma}\right)$-bimodules.

The map $X(v):(n) Q^{\Gamma} \rightarrow(n) Q^{\Gamma}[1]\{-1\}$ is defined by using a resolution of $Q^{\Gamma}$ as above. We consider $V^{\vee} \otimes A_{1}^{\Gamma}$, where $A_{1}^{\Gamma}$ only acts on the second factor from the right. We have the maps

$$
d: V^{\vee} \otimes A_{1}^{\Gamma} \rightarrow A_{1}^{\Gamma} \quad \text { and } \quad d: \bigwedge^{2} V^{\vee} \otimes A_{1}^{\Gamma} \rightarrow V^{\vee} \otimes A_{1}^{\Gamma}
$$

of right $A_{1}^{\Gamma}$-modules via

$$
\begin{aligned}
w \otimes(f, \gamma) & \mapsto(w f, \gamma) \quad \text { and } \\
\left(w_{1} \wedge w_{2}\right) \otimes(f, \gamma) & \mapsto w_{1} \otimes\left(w_{2} f, \gamma\right)-w_{2} \otimes\left(w_{1} f, \gamma\right) .
\end{aligned}
$$

Using these maps we have a free resolution of the right $A_{1}^{\Gamma}$-module $\mathbb{k}[\Gamma]$ as in (12):

$$
0 \rightarrow \bigwedge^{2} V^{\vee} \otimes A_{1}^{\Gamma} \stackrel{d}{\rightarrow} V^{\vee} \otimes A_{1}^{\Gamma} \stackrel{d}{\rightarrow} A_{1}^{\Gamma} \rightarrow \mathbb{k}[\Gamma]
$$

Then we can write a map $X(v): \mathbb{k}[\Gamma] \rightarrow \mathbb{k}[\Gamma][1]\{-1\}$ of graded right $A_{1}^{\Gamma}$ modules as in equation (13), except that we use the maps 
$\psi_{v}: V^{\vee} \otimes A_{1}^{\Gamma} \rightarrow A_{1}^{\Gamma}\{-1\} \quad$ and $\quad \psi_{v}^{\prime}: \bigwedge^{2} V^{\vee} \otimes A_{1}^{\Gamma} \rightarrow V^{\vee} \otimes A_{1}^{\Gamma}\{-1\}$ given by

$$
\begin{aligned}
w \otimes(f, \gamma) & \mapsto-\langle v, w\rangle(f, \gamma) \quad \text { and } \\
\left(w_{1} \wedge w_{2}\right) \otimes(f, \gamma) & \mapsto\left\langle v, w_{1}\right\rangle w_{2} \otimes(f, \gamma)-\left\langle v, w_{2}\right\rangle w_{1} \otimes(f, \gamma) .
\end{aligned}
$$

Using these maps, we can again write down the following commutative diagram:

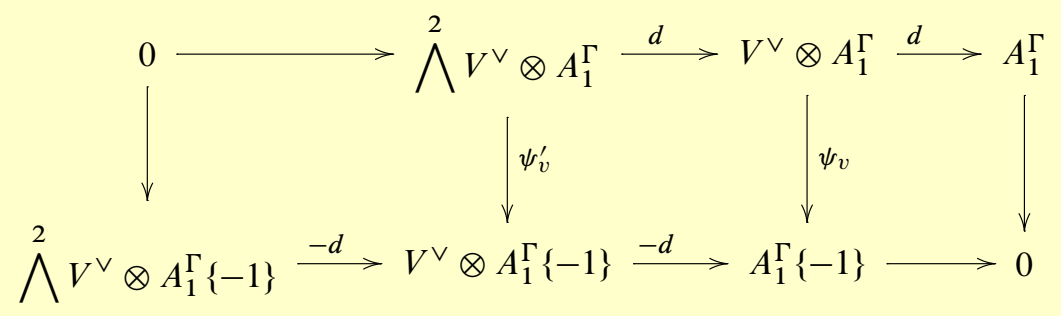

This induces a map $X(v): \mathbb{k}[\Gamma] \rightarrow \mathbb{k}[\Gamma][1]\{-1\}$ and subsequently a map $X(v)$ : (n) $Q^{\Gamma} \rightarrow(n) Q^{\Gamma}[1]\{-1\}$ (in the derived category) of graded $\left(A_{n}^{\Gamma}, A_{n+1}^{\Gamma}\right)$-bimodules.

\subsubsection{Definition of $T \in \operatorname{End}\left(P^{2}\right)$ and $T \in \operatorname{End}\left(Q^{2}\right)$}

To define $T: \mathrm{PP} \rightarrow \mathrm{PP}$, we need a map:

$$
P^{\Gamma}(n+1) \otimes_{A_{n+1}^{\Gamma}} P^{\Gamma}(n) \rightarrow P^{\Gamma}(n+1) \otimes_{A_{n+1}^{\Gamma}} P^{\Gamma}(n) .
$$

Now

$$
\begin{aligned}
& P^{\Gamma}(n+1) \otimes_{A_{n+1}^{\Gamma}} P^{\Gamma}(n) \\
& \quad \cong A_{n+2}^{\Gamma} \otimes_{A_{n+1}^{\Gamma} \otimes A_{1}^{\Gamma}}\left(A_{n+1}^{\Gamma} \otimes \mathbb{k}[\Gamma]\right) \otimes_{A_{n}^{\Gamma} \otimes A_{1}^{\Gamma}}\left(A_{n}^{\Gamma} \otimes \mathbb{k}[\Gamma]\right) \\
& \quad \cong A_{n+2}^{\Gamma} \otimes_{A_{n}^{\Gamma} \otimes A_{1}^{\Gamma} \otimes A_{1}^{\Gamma}}\left(A_{n}^{\Gamma} \otimes \mathbb{k}[\Gamma] \otimes \mathbb{k}[\Gamma]\right) \\
& \quad \cong A_{n+2}^{\Gamma} \otimes_{A_{n}^{\Gamma} \otimes A_{2}^{\Gamma}} A_{n}^{\Gamma} \otimes\left(A_{2}^{\Gamma} \otimes_{A_{1}^{\Gamma} \otimes A_{1}^{\Gamma}} \mathbb{k}[\Gamma] \otimes \mathbb{k}[\Gamma]\right) .
\end{aligned}
$$

Now define a map $T: A_{2}^{\Gamma} \otimes_{A_{1}^{\Gamma} \otimes A_{1}^{\Gamma}}(\mathbb{k}[\Gamma] \otimes \mathbb{k}[\Gamma]) \rightarrow A_{2}^{\Gamma} \otimes_{A_{1}^{\Gamma} \otimes A_{1}^{\Gamma}}(\mathbb{k}[\Gamma] \otimes \mathbb{k}[\Gamma])$ by

$$
a \otimes\left(\gamma \otimes \gamma^{\prime}\right) \mapsto a s_{1} \otimes\left(\gamma^{\prime} \otimes \gamma\right),
$$

where $a \in A_{2}^{\Gamma}$ and $s_{1} \in S_{2}$ is the transposition $(1,2)$.

Let us check that this induces a well-defined map. Suppose that $a=\left(a^{\prime}, a^{\prime \prime}\right)$, where $a^{\prime}, a^{\prime \prime} \in A_{1}^{\Gamma}$. Then 


$$
T\left(a \otimes\left(\gamma \otimes \gamma^{\prime}\right)\right)=T\left(1 \otimes\left(a^{\prime} \cdot \gamma \otimes a^{\prime \prime} \cdot \gamma^{\prime}\right)\right)=s_{1} \otimes\left(a^{\prime \prime} \gamma^{\prime} \otimes a^{\prime} \gamma\right)
$$

On the other hand,

$$
\left(a^{\prime}, a^{\prime \prime}\right) s_{1} \otimes\left(\gamma^{\prime} \otimes \gamma\right)=s_{1}\left(a^{\prime \prime}, a^{\prime}\right) \otimes\left(\gamma^{\prime} \otimes \gamma\right)=s_{1} \otimes\left(a^{\prime \prime} \gamma^{\prime}, a^{\prime} \gamma\right)
$$

which agrees with (17). This shows that $T$ is well defined.

Notice that the induced map

$$
\begin{aligned}
T: & A_{n+2}^{\Gamma} \otimes_{A_{n}^{\Gamma} \otimes A_{1}^{\Gamma} \otimes A_{1}^{\Gamma}}\left(A_{n}^{\Gamma} \otimes \mathbb{k}[\Gamma] \otimes \mathbb{k}[\Gamma]\right) \\
& \rightarrow A_{n+2}^{\Gamma} \otimes_{A_{n}^{\Gamma} \otimes A_{1}^{\Gamma} \otimes A_{1}^{\Gamma}}\left(A_{n}^{\Gamma} \otimes \mathbb{k}[\Gamma] \otimes \mathbb{k}[\Gamma]\right)
\end{aligned}
$$

is given by

$$
1 \otimes\left(1 \otimes \gamma \otimes \gamma^{\prime}\right) \mapsto s_{n+1} \otimes\left(1 \otimes \gamma^{\prime} \otimes \gamma\right),
$$

where $s_{n+1}=(n+1, n+2)$.

The map $T: \mathrm{QQ} \rightarrow \mathrm{QQ}$ is defined in exactly the same way.

\subsubsection{Definition of $T: Q P \rightarrow P Q$}

To define $T: \mathrm{QP} \rightarrow \mathrm{PQ}$, we need a map,

$$
\begin{aligned}
& \left(A_{n}^{\Gamma} \otimes \mathbb{k}[\Gamma]\right) \otimes_{A_{n}^{\Gamma} \otimes A_{1}^{\Gamma}} A_{n+1}^{\Gamma} \otimes_{A_{n}^{\Gamma} \otimes A_{1}^{\Gamma}}\left(A_{n}^{\Gamma} \otimes \mathbb{k}[\Gamma]\right) \\
& \quad \rightarrow A_{n}^{\Gamma} \otimes_{A_{n-1}^{\Gamma} \otimes A_{1}^{\Gamma}}\left(A_{n-1}^{\Gamma} \otimes \mathbb{k}[\Gamma] \otimes \mathbb{k}[\Gamma]\right) \otimes_{A_{n-1}^{\Gamma} \otimes A_{1}^{\Gamma}} A_{n}^{\Gamma},
\end{aligned}
$$

of $\left(A_{n}^{\Gamma}, A_{n}^{\Gamma}\right)$-bimodules. To define this map, it suffices to determine where to take $(1 \otimes \gamma) \otimes a \otimes\left(1 \otimes \gamma^{\prime}\right)$, where $a \in A_{n+1}^{\Gamma}$ equals 1 or $s_{n}=(n, n+1)$. If $a=1$, then we map it to zero, while

$$
(1 \otimes \gamma) \otimes s_{n} \otimes\left(1 \otimes \gamma^{\prime}\right) \mapsto 1 \otimes\left(1 \otimes \gamma^{\prime} \otimes \gamma\right) \otimes 1 .
$$

\subsubsection{Definition of $T: P Q \rightarrow Q P$}

To define $T: \mathrm{PQ} \rightarrow \mathrm{QP}$, we need a map,

$$
\begin{aligned}
& A_{n}^{\Gamma} \otimes_{A_{n-1}^{\Gamma} \otimes A_{1}^{\Gamma}}\left(A_{n-1}^{\Gamma} \otimes \mathbb{k}[\Gamma] \otimes \mathbb{k}[\Gamma]\right) \otimes_{A_{n-1}^{\Gamma} \otimes A_{1}^{\Gamma}} A_{n}^{\Gamma} \\
& \quad \rightarrow\left(A_{n}^{\Gamma} \otimes \mathbb{k}[\Gamma]\right) \otimes_{A_{n}^{\Gamma} \otimes A_{1}^{\Gamma}} A_{n+1}^{\Gamma} \otimes_{A_{n}^{\Gamma} \otimes A_{1}^{\Gamma}}\left(A_{n}^{\Gamma} \otimes \mathbb{k}[\Gamma]\right),
\end{aligned}
$$

of $\left(A_{n}^{\Gamma}, A_{n}^{\Gamma}\right)$-bimodules. This map is uniquely defined by

$1 \otimes(1 \otimes 1 \otimes 1) \otimes 1 \mapsto(1 \otimes 1) \otimes s_{n} \otimes(1 \otimes 1)$. 
4.3.7. Definition of adj: $Q P \rightarrow \mathrm{id}[-1]\{1\}$

We need a map of $\left(A_{n}^{\Gamma}, A_{n}^{\Gamma}\right)$-bimodules:

$$
\text { adj : (n) } Q^{\Gamma} \otimes_{A_{n+1}^{\Gamma}} P^{\Gamma}(n) \rightarrow A_{n}^{\Gamma} .
$$

Now

$$
(n) Q^{\Gamma} \otimes_{A_{n+1}^{\Gamma}} P^{\Gamma}(n)=\left(A_{n}^{\Gamma} \otimes \mathbb{k}[\Gamma]\right) \otimes_{A_{n}^{\Gamma} \otimes A_{1}^{\Gamma}} A_{n+1}^{\Gamma} \otimes_{A_{n}^{\Gamma} \otimes A_{1}^{\Gamma}}\left(A_{n}^{\Gamma} \otimes \mathbb{k}[\Gamma]\right) .
$$

Notice that $\mathbb{k}\left[S_{n+1}\right]$ as a $\mathbb{k}\left[S_{n}\right]$-bimodule is free and generated by 1 and $s_{n}=$ $(n, n+1)$. So the map $\mathbb{k}\left[S_{n+1}\right] \rightarrow \mathbb{k}\left[S_{n}\right]$ of $\mathbb{k}\left[S_{n}\right]$-bimodules given by $1 \mapsto 1$ and $s_{n} \mapsto 0$ induces a map $A_{n+1}^{\Gamma} \rightarrow A_{n}^{\Gamma} \otimes A_{1}^{\Gamma}$ of $\left(A_{n}^{\Gamma} \otimes A_{1}^{\Gamma}\right)$-bimodules. Subsequently, we obtain a map:

$$
\begin{aligned}
\text { (n) } Q^{\Gamma} & \otimes_{A_{n+1}^{\Gamma}} P^{\Gamma}(n) \\
& \rightarrow\left(A_{n}^{\Gamma} \otimes \mathbb{k}[\Gamma]\right) \otimes_{A_{n}^{\Gamma} \otimes A_{1}^{\Gamma}}\left(A_{n}^{\Gamma} \otimes A_{1}^{\Gamma}\right) \otimes_{A_{n}^{\Gamma} \otimes A_{1}^{\Gamma}}\left(A_{n}^{\Gamma} \otimes \mathbb{k}[\Gamma]\right) \\
& \cong\left(A_{n}^{\Gamma} \otimes \mathbb{k}[\Gamma]\right) \otimes_{A_{n}^{\Gamma} \otimes A_{1}^{\Gamma}}\left(A_{n}^{\Gamma} \otimes \mathbb{k}[\Gamma]\right) .
\end{aligned}
$$

Using the composition $\mathbb{k}[\Gamma] \otimes_{A_{1}^{\Gamma}} \mathbb{k}[\Gamma] \rightarrow \mathbb{k}[\Gamma] \stackrel{\text { tr }}{\rightarrow} \mathbb{k}$, where the first map is multiplication and the second is the trace map (normalized so that $\operatorname{tr}(1)=1$ ), we get a morphism:

$$
\left(A_{n}^{\Gamma} \otimes \mathbb{k}[\Gamma]\right) \otimes_{A_{n}^{\Gamma} \otimes A_{1}^{\Gamma}}\left(A_{n}^{\Gamma} \otimes \mathbb{k}[\Gamma]\right) \rightarrow A_{n}^{\Gamma} \otimes_{A_{n}^{\Gamma}} A_{n}^{\Gamma}=A_{n}^{\Gamma} .
$$

Composing with (18) defines adj : $(n) Q^{\Gamma} \otimes_{A_{n+1}^{\Gamma}} P^{\Gamma}(n) \rightarrow A_{n}^{\Gamma}$.

\subsubsection{Definition of adj : $P Q \rightarrow \mathrm{id}[1]\{-1\}$}

We need a map of $\left(A_{n+1}^{\Gamma}, A_{n+1}^{\Gamma}\right)$-bimodules:

$$
P^{\Gamma}(n) \otimes_{A_{n}^{\Gamma}}(n) Q^{\Gamma} \rightarrow A_{n+1}^{\Gamma}[2]\{-2\} .
$$

Now

$$
P^{\Gamma}(n) \otimes_{A_{n}^{\Gamma}}(n) Q^{\Gamma}=A_{n+1}^{\Gamma} \otimes_{A_{n}^{\Gamma} \otimes A_{1}^{\Gamma}}\left(A_{n}^{\Gamma} \otimes \mathbb{k}[\Gamma] \otimes \mathbb{k}[\Gamma]\right) \otimes_{A_{n}^{\Gamma} \otimes A_{1}^{\Gamma}} A_{n+1}^{\Gamma},
$$

so we basically need a map $h: \mathbb{k}[\Gamma] \otimes \mathbb{k}[\Gamma] \rightarrow A_{1}^{\Gamma}[2]\{-2\}$ of graded $\left(A_{1}^{\Gamma}, A_{1}^{\Gamma}\right)$ bimodules. Then we define adj as the composition

$$
\begin{aligned}
\operatorname{adj}: & A_{n+1}^{\Gamma} \otimes_{A_{n}^{\Gamma} \otimes A_{1}^{\Gamma}}\left(A_{n}^{\Gamma} \otimes \mathbb{k}[\Gamma] \otimes \mathbb{k}[\Gamma]\right) \otimes_{A_{n}^{\Gamma} \otimes A_{1}^{\Gamma}} A_{n+1}^{\Gamma} \\
& \stackrel{h}{\rightarrow} A_{n+1}^{\Gamma} \otimes_{A_{n}^{\Gamma} \otimes A_{1}^{\Gamma}} A_{n+1}^{\Gamma}[2]\{-2\} \rightarrow A_{n+1}^{\Gamma}[2]\{-2\},
\end{aligned}
$$

where the second map is multiplication. 
To define $h$ we use the resolutions (12) and (14) of $\mathbb{k}[\Gamma]$. Tensoring the two resolutions we see that $h$ is defined by a map:

$\left(A_{1}^{\Gamma} \otimes \bigwedge^{2} V^{\vee}\right) \otimes A_{1}^{\Gamma} \oplus\left(A_{1}^{\Gamma} \otimes V^{\vee}\right) \otimes\left(V^{\vee} \otimes A_{1}^{\Gamma}\right) \oplus A_{1}^{\Gamma} \otimes\left(\bigwedge^{2} V^{\vee} \otimes A_{1}^{\Gamma}\right) \rightarrow A_{1}^{\Gamma}\{-2\}$.

We define the map from each summand as

(1) $\quad\left((f, \gamma) \otimes\left(w \wedge w^{\prime}\right)\right) \otimes\left(f^{\prime}, \gamma^{\prime}\right) \mapsto(f, \gamma)\left(f^{\prime}, \gamma^{\prime}\right) \omega\left(w \wedge w^{\prime}\right)$,

(2) $\quad((f, \gamma) \otimes w) \otimes\left(w^{\prime} \otimes\left(f^{\prime}, \gamma^{\prime}\right)\right) \mapsto(f, \gamma)\left(f^{\prime}, \gamma^{\prime}\right) \omega\left(w \wedge w^{\prime}\right)$,

(3) $\quad(f, \gamma) \otimes\left(\left(w \wedge w^{\prime}\right) \otimes\left(f^{\prime}, \gamma^{\prime}\right)\right) \mapsto-(f, \gamma)\left(f^{\prime}, \gamma^{\prime}\right) \omega\left(w \wedge w^{\prime}\right)$, where $\omega: \bigwedge^{2} V^{\vee} \rightarrow \mathbb{k}$ is our fixed isomorphism.

In order for this map to be well defined (i.e., a map of complexes), we need the composition

$$
\begin{gathered}
\left(A_{1}^{\Gamma} \otimes \bigwedge^{2} V^{\vee}\right) \otimes A_{1}^{\Gamma} \oplus\left(A_{1}^{\Gamma} \otimes V^{\vee}\right) \otimes\left(V^{\vee} \otimes A_{1}^{\Gamma}\right) \oplus A_{1}^{\Gamma} \otimes\left(\bigwedge^{2} V^{\vee} \otimes A_{1}^{\Gamma}\right) \longrightarrow \\
\uparrow \\
\left(A_{1}^{\Gamma} \otimes \bigwedge^{2} V^{\vee}\right) \otimes\left(V^{\vee} \otimes A_{1}^{\Gamma}\right) \oplus\left(A_{1}^{\Gamma} \otimes V^{\vee}\right) \otimes\left(\bigwedge^{2} V^{\vee} \otimes A_{1}^{\Gamma}\right)
\end{gathered}
$$

to be zero.

Let us check that the composition $\left(A_{1}^{\Gamma} \otimes \bigwedge^{2} V^{\vee}\right) \otimes\left(V^{\vee} \otimes A_{1}^{\Gamma}\right) \rightarrow A_{1}^{\Gamma}\{-2\}$ is zero. The first map is

$$
\begin{aligned}
(f, \gamma) \otimes\left(w \wedge w^{\prime}\right) \otimes\left(w^{\prime \prime} \otimes\left(f^{\prime}, \gamma^{\prime}\right)\right) \mapsto & \left((f, \gamma) \otimes\left(w \wedge w^{\prime}\right)\right) \otimes\left(f^{\prime} w^{\prime \prime}, \gamma^{\prime}\right) \\
& +\left((f \gamma \cdot w, \gamma) \otimes w^{\prime}\right) \otimes\left(w^{\prime \prime} \otimes\left(f^{\prime}, \gamma^{\prime}\right)\right) \\
& -\left(\left(f \gamma \cdot w^{\prime}, \gamma\right) \otimes w\right) \otimes\left(w^{\prime \prime} \otimes\left(f^{\prime}, \gamma^{\prime}\right)\right) .
\end{aligned}
$$

Composing with the second map we get

$$
\begin{aligned}
& \left(f\left(\gamma f^{\prime}\right)\left(\gamma w^{\prime \prime}\right), \gamma \gamma^{\prime}\right) \omega\left(w \wedge w^{\prime}\right) \\
& \quad+\left(f\left(\gamma f^{\prime}\right)(\gamma w), \gamma \gamma^{\prime}\right) \omega\left(w^{\prime} \wedge w^{\prime \prime}\right)-\left(f\left(\gamma f^{\prime}\right)\left(\gamma w^{\prime}\right), \gamma \gamma^{\prime}\right) \omega\left(w \wedge w^{\prime \prime}\right) .
\end{aligned}
$$

Since the maps are linear in the $w$ 's, it suffices to check that this is zero when $w=w^{\prime}$, when $w=w^{\prime \prime}$, and when $w^{\prime}=w^{\prime \prime}$. In all cases, one of the three terms is immediately zero and the other two cancel out.

Similarly, the other composition maps $((f, \gamma) \otimes w) \otimes\left(\left(w^{\prime} \wedge w^{\prime \prime}\right) \otimes\left(f^{\prime}, \gamma^{\prime}\right)\right)$ to

$$
\begin{aligned}
& -\left(f(\gamma w)\left(\gamma f^{\prime}\right), \gamma \gamma^{\prime}\right) \omega\left(w^{\prime} \wedge w^{\prime \prime}\right) \\
& \quad-\left(f\left(\gamma w^{\prime \prime}\right)\left(\gamma f^{\prime}\right), \gamma \gamma^{\prime}\right) \omega\left(w \wedge w^{\prime}\right)+\left(f\left(\gamma w^{\prime}\right)\left(\gamma f^{\prime}\right), \gamma \gamma^{\prime}\right) \omega\left(w \wedge w^{\prime \prime}\right),
\end{aligned}
$$

which also equals zero. 
4.3.9. Definition of adj : id $\rightarrow Q P[-1]\{1\}$

We need a map of $\left(A_{n}^{\Gamma}, A_{n}^{\Gamma}\right)$-bimodules,

$$
A_{n}^{\Gamma} \rightarrow(n) Q^{\Gamma} \otimes_{A_{n+1}^{\Gamma}} P^{\Gamma}(n)[-2]\{2\},
$$

which translates to a map,

$$
A_{n}^{\Gamma}[2]\{-2\} \rightarrow\left(A_{n}^{\Gamma} \otimes \mathbb{k}[\Gamma]\right) \otimes_{A_{n}^{\Gamma} \otimes A_{1}^{\Gamma}} A_{n+1}^{\Gamma} \otimes_{A_{1}^{\Gamma} \otimes A_{n}^{\Gamma}}\left(\mathbb{k}[\Gamma] \otimes A_{n}^{\Gamma}\right) .
$$

We first replace each $\mathbb{k}[\Gamma]$ with its resolution using (12) and (14). Then to define the map in (20) we just need to define a map,

$$
\begin{aligned}
A_{n}^{\Gamma}\{-2\} \rightarrow & \bigoplus_{k+l=2}\left(A_{n}^{\Gamma} \otimes\left(\bigwedge^{k} V^{\vee} \otimes A_{1}^{\Gamma}\right)\right) \otimes_{A_{n}^{\Gamma} \otimes A_{1}^{\Gamma}} A_{n+1}^{\Gamma} \\
& \otimes_{A_{1}^{\Gamma} \otimes A_{n}^{\Gamma}}\left(\left(A_{1}^{\Gamma} \otimes \bigwedge^{l} V^{\vee}\right) \otimes A_{n}^{\Gamma}\right),
\end{aligned}
$$

of graded $\left(A_{n}^{\Gamma}, A_{n}^{\Gamma}\right)$-bimodules. We send

$$
\begin{aligned}
1 \mapsto(1 \otimes 1) & \otimes 1 \otimes\left(\left(1 \otimes w_{1} \wedge w_{2}\right) \otimes 1\right) \\
& -\left(1 \otimes\left(w_{1} \otimes 1\right)\right) \otimes 1 \otimes\left(\left(1 \otimes w_{2}\right) \otimes 1\right) \\
& +\left(1 \otimes\left(w_{2} \otimes 1\right)\right) \otimes 1 \otimes\left(\left(1 \otimes w_{1}\right) \otimes 1\right) \\
& -\left(1 \otimes\left(w_{1} \wedge w_{2} \otimes 1\right)\right) \otimes 1 \otimes(1 \otimes 1),
\end{aligned}
$$

where $w_{1}, w_{2}$ is our chosen basis of $V^{\vee}$. This uniquely determines the map.

To see that this is well defined (i.e., a map of complexes), we need to check that the composition

$$
\begin{array}{r}
\bigoplus_{k+l=1}\left(A_{n}^{\Gamma} \otimes\left(\bigwedge^{k} V^{\vee} \otimes A_{1}^{\Gamma}\right)\right) \otimes_{A_{n}^{\Gamma} \otimes A_{1}^{\Gamma}} A_{n+1}^{\Gamma} \otimes_{A_{1}^{\Gamma} \otimes A_{n}^{\Gamma}}\left(\left(A_{1}^{\Gamma} \otimes \bigwedge^{l} V^{\vee}\right) \otimes A_{n}^{\Gamma}\right) \\
A_{n}^{\Gamma}\{-2\} \longrightarrow \\
\bigoplus_{k+l=2}\left(A_{n}^{\Gamma} \otimes\left(\bigwedge^{k} V^{\vee} \otimes A_{1}^{\Gamma}\right)\right) \otimes_{A_{n}^{\Gamma} \otimes A_{1}^{\Gamma}} A_{n+1}^{\Gamma} \otimes_{A_{1}^{\Gamma} \otimes A_{n}^{\Gamma}}\left(\left(A_{1}^{\Gamma} \otimes \bigwedge^{l} V^{\vee}\right) \otimes A_{n}^{\Gamma}\right)
\end{array}
$$

is zero. The check of this fact is a straightforward exercise.

4.3.10. Definition of adj : id $\rightarrow P Q[1]\{-1\}$

We need a map of graded $\left(A_{n+1}^{\Gamma}, A_{n+1}^{\Gamma}\right)$-bimodules $A_{n+1}^{\Gamma} \rightarrow P^{\Gamma}(n) \otimes_{A_{n}^{\Gamma}}(n) Q^{\Gamma}$ or, equivalently, a map: 


$$
A_{n+1}^{\Gamma} \rightarrow A_{n+1}^{\Gamma} \otimes_{A_{n}^{\Gamma} \otimes A_{1}^{\Gamma}}\left(A_{n}^{\Gamma} \otimes \mathbb{k}[\Gamma] \otimes \mathbb{k}[\Gamma]\right) \otimes_{A_{n}^{\Gamma} \otimes A_{1}^{\Gamma}} A_{n+1}^{\Gamma} .
$$

We send

$$
1 \mapsto \sum_{i=0}^{n} \sum_{\gamma \in \Gamma} s_{i} \cdots s_{n} \otimes\left(1 \otimes \gamma \otimes \gamma^{-1}\right) \otimes s_{n} \cdots s_{i},
$$

where $s_{i}=(i, i+1) \in S_{n+1}$. Here $s_{i} \cdots s_{n}=1$, if $i=0$ by convention.

To check that this bimodule map is well defined, we need to show that

$$
\begin{aligned}
& \sum_{i=0}^{n} \sum_{\gamma \in \Gamma} a s_{i} \cdots s_{n} \otimes\left(1 \otimes \gamma \otimes \gamma^{-1}\right) \otimes s_{n} \cdots s_{i} \\
& \quad=\sum_{i=0}^{n} \sum_{\gamma \in \Gamma} s_{i} \cdots s_{n} \otimes\left(1 \otimes \gamma \otimes \gamma^{-1}\right) \otimes s_{n} \cdots s_{i} a
\end{aligned}
$$

for any $a \in A_{n+1}^{\Gamma}$. If $a \in\left(A_{1}^{\Gamma}\right)^{\otimes n+1} \subset A_{n+1}^{\Gamma}$, then

$$
\begin{aligned}
\sum_{i=0}^{n} \sum_{\gamma \in \Gamma} a s_{i} \cdots s_{n} \otimes\left(1 \otimes \gamma \otimes \gamma^{-1}\right) \otimes s_{n} \cdots s_{i} \\
\quad=\sum_{i=0}^{n} \sum_{\gamma \in \Gamma} s_{i} \cdots s_{n} b_{i} \otimes\left(1 \otimes \gamma \otimes \gamma^{-1}\right) \otimes s_{n} \cdots s_{i} \\
\quad=\sum_{i=0}^{n} \sum_{\gamma \in \Gamma} s_{i} \cdots s_{n} \otimes\left(1 \otimes \gamma \otimes \gamma^{-1}\right) \otimes b_{i} s_{n} \cdots s_{i} \\
=\sum_{i=0}^{n} \sum_{\gamma \in \Gamma} s_{i} \cdots s_{n} \otimes\left(1 \otimes \gamma \otimes \gamma^{-1}\right) \otimes s_{n} \cdots s_{i} a,
\end{aligned}
$$

where $b_{i}=\left(s_{n} \cdots s_{i}\right) \cdot a$. The second equality follows since $\sum_{\gamma \in \Gamma} \gamma \otimes \gamma^{-1}$ lies in the center of $\mathbb{k}[\Gamma] \otimes_{\mathbb{k}} \mathbb{k}[\Gamma]$.

Since $A_{n+1}^{\Gamma}$ is generated by $\mathbb{k}\left[S_{n+1}\right]$ as an $\left(\left(A_{1}^{\Gamma}\right)^{\otimes n+1},\left(A_{1}^{\Gamma}\right)^{\otimes n+1}\right)$-bimodule, it remains to show that

$$
\begin{aligned}
& \sum_{i=0}^{n} \sum_{\gamma \in \Gamma} s_{k} s_{i} \cdots s_{n} \otimes\left(1 \otimes \gamma \otimes \gamma^{-1}\right) \otimes s_{n} \cdots s_{i} \\
& \quad=\sum_{i=0}^{n} \sum_{\gamma \in \Gamma} s_{i} \cdots s_{n} \otimes\left(1 \otimes \gamma \otimes \gamma^{-1}\right) \otimes s_{n} \cdots s_{i} s_{k}
\end{aligned}
$$

for $k=1, \ldots, n$. The left-hand side is the sum over $\gamma \in \Gamma$ of 


$$
\begin{aligned}
& \sum_{i=0}^{k-1} s_{i} \cdots s_{n} s_{k-1} \otimes\left(1 \otimes \gamma \otimes \gamma^{-1}\right) \otimes s_{n} \cdots s_{i} \\
& \quad+s_{k} s_{k+1} \cdots s_{n} \otimes\left(1 \otimes \gamma \otimes \gamma^{-1}\right) \otimes s_{n} \cdots s_{k+1} \\
& \quad+s_{k+1} \cdots s_{n} \otimes\left(1 \otimes \gamma \otimes \gamma^{-1}\right) \otimes s_{n} \cdots s_{k} \\
& \quad+\sum_{i=k+2}^{n} s_{i} \cdots s_{n} s_{k} \otimes\left(1 \otimes \gamma \otimes \gamma^{-1}\right) \otimes s_{n} \cdots s_{i}
\end{aligned}
$$

because $s_{k} s_{i} \cdots s_{n}=s_{i} \cdots s_{n} s_{k-1}$ if $i \leq k-1$ and $s_{k} s_{i} \cdots s_{n}=s_{i} \cdots s_{n} s_{k}$ if $i \geq k+2$. A similar calculation of the right-hand side yields the same expression, so adj is well defined.

\subsection{Second main theorem}

\section{THEOREM 2}

The natural transformations $X, T$, and adj satisfy the Heisenberg 2-relations and give a categorical Heisenberg action of $\mathscr{H}_{\Gamma}$ on $\bigoplus_{n \geq 0} D\left(A_{n}^{\Gamma}-\mathrm{gmod}\right)$.

We shall check all the Heisenberg relations required for the proof of Theorem 2 in the next section.

\section{Remark 4}

In type $A$ (when $\Gamma$ is a cyclic group), one can define everything $\mathbb{k}^{\times}$-equivariantly, where the $\mathbb{k}^{\times} \subset \operatorname{SL}(V)$ is the torus which commutes with $\Gamma$. More precisely, the action of $\mathbb{k}^{\times}$on $V$ induces an action on $\operatorname{Sym}^{*}\left(V^{\vee}\right)$, and instead of $A_{n}^{\Gamma}$ one uses

$$
\begin{aligned}
\hat{A}_{n}^{\Gamma}:= & {\left[\left(\operatorname{Sym}^{*}\left(V^{\vee}\right) \rtimes\left(\Gamma \times \mathbb{k}^{\times}\right)\right) \otimes\left(\operatorname{Sym}^{*}\left(V^{\vee}\right) \rtimes\left(\Gamma \times \mathbb{k}^{\times}\right)\right) \otimes \cdots\right.} \\
\otimes & \left.\left(\operatorname{Sym}^{*}\left(V^{\vee}\right) \rtimes\left(\Gamma \times \mathbb{k}^{\times}\right)\right)\right] \rtimes S_{n} .
\end{aligned}
$$

It is easy to check that all the 1-morphisms and 2-morphisms defined above are compatible with this extra $\mathbb{k}^{\times}$-structure. This means that Theorem 2 still holds, so we get an action of $\mathscr{H}_{\Gamma}$ on $\bigoplus_{n \geq 0} D\left(\hat{A}_{n}^{\Gamma}-\right.$ gmod) (see also [10]).

\section{Proof of Theorem 2}

\subsection{Composition of $X$ 's}

We can define $X(b): \mathrm{P} \rightarrow \mathrm{P}[|b|]\{-|b|\}$ for any homogeneous $b \in B^{\Gamma}$ by composing a sequence of $X(\gamma)$ and $X(v)$, where $\gamma \in \Gamma$ and $v \in V$. In order for this to be well defined, we need to check that

(1) $\quad X(\gamma) X\left(\gamma^{\prime}\right)=X\left(\gamma \gamma^{\prime}\right)$ for any $\gamma, \gamma^{\prime} \in \Gamma$; 
(2) $\quad X(\gamma) X(v)=X(\gamma \cdot v) X(\gamma)$ for any $\gamma \in \Gamma$ and $v \in V$; and

(3) $\quad X\left(v^{\prime}\right) X(v)=-X(v) X\left(v^{\prime}\right)$.

(1) The first assertion is clear since $\Gamma$ acts on $P^{\Gamma}(n)=A_{n+1} \otimes_{A_{n} \otimes A_{1}}\left(A_{n} \otimes\right.$ $\mathbb{k}[\Gamma])$ by right multiplication on $\mathbb{k}[\Gamma]$.

(2) To see the second assertion we replace $\mathbb{k}[\Gamma]$ by its free resolution (12). In the free resolution, $\Gamma$ acts on the right only on the first factor of $A_{1}^{\Gamma} \otimes \bigwedge^{i} V^{\vee}$. Writing out the composition we see that two pairs of compositions should agree:

- $\quad A_{1}^{\Gamma} \otimes V^{\vee} \stackrel{\gamma}{\rightarrow} A_{1}^{\Gamma} \otimes V^{\vee} \stackrel{\phi_{v}}{\rightarrow} A_{1}^{\Gamma}\{-1\}$ and $A_{1}^{\Gamma} \otimes V^{\vee} \stackrel{\phi_{\gamma v}}{\rightarrow} A_{1}^{\Gamma}\{-1\} \stackrel{\gamma}{\rightarrow} A_{1}^{\Gamma}\{-1\} ;$

- $\quad A_{1}^{\Gamma} \otimes \bigwedge^{2} V^{\vee} \stackrel{\gamma}{\rightarrow} A_{1}^{\Gamma} \otimes \bigwedge^{2} V^{\vee} \stackrel{\phi_{v}^{\prime}}{\rightarrow} A_{1}^{\Gamma} \otimes V^{\vee}\{-1\}$ and $A_{1}^{\Gamma} \otimes \bigwedge^{2} V^{\vee} \stackrel{\phi_{\gamma v}^{\prime}}{\longrightarrow}$ $A_{1}^{\Gamma} \otimes V^{\vee}\{-1\} \stackrel{\gamma}{\rightarrow} A_{1}^{\Gamma} \otimes V^{\vee}\{-1\}$.

In the first pair, the first composition is equal to

$$
\left(f, \gamma^{\prime}\right) \otimes w \mapsto\left(f, \gamma^{\prime} \gamma\right) \otimes\left(\gamma^{-1} w\right) \mapsto\left\langle\gamma^{-1} w, v\right\rangle\left(f, \gamma^{\prime} \gamma\right)
$$

while the second composition is equal to

$$
\left(f, \gamma^{\prime}\right) \otimes w \mapsto\langle w, \gamma v\rangle\left(f, \gamma^{\prime}\right) \mapsto\langle w, \gamma v\rangle\left(f, \gamma^{\prime} \gamma\right)
$$

and these are the same since $\langle\cdot, \cdot\rangle$ is invariant under the action of $\Gamma$.

Similarly, in the second pair, the first composition is equal to

$$
\begin{aligned}
\left(f, \gamma^{\prime}\right) \otimes\left(w_{1} \wedge w_{2}\right) \mapsto & \left(f, \gamma^{\prime} \gamma\right) \otimes\left(\gamma^{-1} w_{1} \wedge \gamma^{-1} w_{2}\right) \\
\mapsto & -\left\langle\gamma^{-1} w_{2}, v\right\rangle\left(f, \gamma^{\prime} \gamma\right) \otimes\left(\gamma^{-1} w_{1}\right) \\
& +\left\langle\gamma^{-1} w_{1}, v\right\rangle\left(f, \gamma^{\prime} \gamma\right) \otimes\left(\gamma^{-1} w_{2}\right),
\end{aligned}
$$

while the second composition is

$$
\begin{aligned}
\left(f, \gamma^{\prime}\right) \otimes\left(w_{1} \wedge w_{2}\right) \mapsto & -\left\langle w_{2}, \gamma v\right\rangle\left(f, \gamma^{\prime}\right) \otimes w_{1}+\left\langle w_{1}, \gamma v\right\rangle\left(f, \gamma^{\prime}\right) \otimes w_{2} \\
\mapsto & -\left\langle w_{2}, \gamma v\right\rangle\left(f, \gamma^{\prime} \gamma\right) \otimes\left(\gamma^{-1} w_{1}\right) \\
& +\left\langle w_{1}, \gamma v\right\rangle\left(f, \gamma^{\prime} \gamma\right) \otimes\left(\gamma^{-1} w_{2}\right),
\end{aligned}
$$

which is clearly the same as the first composition.

(3) To see the third assertion, we again replace $\mathbb{k}[\Gamma]$ by its free resolution (12) and then we need to show that the compositions

$$
\begin{aligned}
& A_{1}^{\Gamma} \otimes \bigwedge^{2} V^{\vee} \stackrel{\phi_{v^{\prime}}^{\prime}}{\longrightarrow} A_{1}^{\Gamma} \otimes V^{\vee}\{-1\} \stackrel{\phi_{v}}{\longrightarrow} A_{1}^{\Gamma}\{-2\} \quad \text { and } \\
& A_{1}^{\Gamma} \otimes \bigwedge^{2} V^{\vee} \stackrel{\phi_{v}^{\prime}}{\longrightarrow} A_{1}^{\Gamma} \otimes V^{\vee}\{-1\} \stackrel{\phi_{v^{\prime}}}{\longrightarrow} A_{1}^{\Gamma}\{-2\}
\end{aligned}
$$


differ precisely by a minus sign. The first composition is

$$
\begin{aligned}
(f, \gamma) \otimes\left(w_{1} \wedge w_{2}\right) & \mapsto-\left\langle w_{2}, v^{\prime}\right\rangle(f, \gamma) \otimes w_{1}+\left\langle w_{1}, v^{\prime}\right\rangle(f, \gamma) \otimes w_{2} \\
& \mapsto-\left\langle w_{1}, v\right\rangle\left\langle w_{2}, v^{\prime}\right\rangle(f, \gamma)+\left\langle w_{2}, v\right\rangle\left\langle w_{1}, v^{\prime}\right\rangle(f, \gamma),
\end{aligned}
$$

and, similarly, the second composition is

$$
(f, \gamma) \otimes\left(w_{1} \wedge w_{2}\right) \mapsto-\left\langle w_{1}, v^{\prime}\right\rangle\left\langle w_{2}, v\right\rangle(f, \gamma)+\left\langle w_{2}, v^{\prime}\right\rangle\left\langle w_{1}, v\right\rangle(f, \gamma) .
$$

These two compositions clearly differ by multiplication by -1 .

This shows that $B^{\Gamma}=\Lambda^{*}(V) \rtimes \Gamma$ acts on $\mathrm{P}$, and a similar proof shows that it also acts on $Q$.

\subsection{1. $X$ 's on different strands}

We also need to check that elements of $B^{\Gamma}$ on different strands supercommute, namely that

$$
(I X(b))\left(X\left(b^{\prime}\right) I\right)=(-1)^{|b|\left|b^{\prime}\right|}\left(X\left(b^{\prime}\right) I\right)(I X(b)) \text { for any homogeneous } b, b^{\prime} \in B^{\Gamma} .
$$

This follows from the discussion at the beginning of this section, since $X(b)$ is a map of degree $|b|$ of complexes; whenever $f: A_{\bullet} \rightarrow A_{\bullet+|f|}$ and $g: A_{\bullet}^{\prime} \rightarrow A_{\bullet+|g|}^{\prime}$ are maps of complexes, it follows that $(f \otimes 1)(1 \otimes g)=(-1)^{|f||g|}(1 \otimes g)(f \otimes 1)$ as maps of complexes $A_{\bullet} \otimes B_{\bullet} \rightarrow A_{\bullet} \otimes B_{\bullet}[|f|+|g|]$.

\subsection{Adjoint relations}

We now check that the following compositions involving adjunctions are all equal to the identity:

(2) $\mathrm{P} \stackrel{\text { adj } I}{\longrightarrow} \mathrm{PQP}[1]\{-1\} \stackrel{I \text { adj }}{\longrightarrow} \mathrm{P}$ and $\mathrm{Q} \stackrel{I \text { adj }}{\longrightarrow} \mathrm{QPQ}[1]\{-1\} \stackrel{\operatorname{adj} I}{\longrightarrow} \mathrm{Q}$.

This will prove the graphical relations

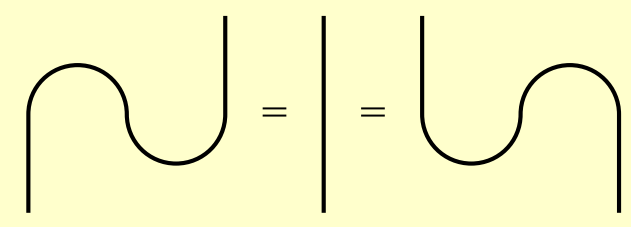

in both possible orientations.

5.2.1. The composition $\mathrm{P} \stackrel{\text { Iadj }}{\longrightarrow} \mathrm{PQP}[-1]\{1\} \stackrel{\text { adj } I}{\longrightarrow} \mathrm{P}$

To show that this is the identity, it suffices to check that the composition $C_{i} \rightarrow$ $D_{i}\{2\} \rightarrow C_{i}$ is the identity for $i=0,1,2$, where 


$$
\begin{aligned}
C_{i}:= & A_{n+1}^{\Gamma} \otimes_{A_{n}^{\Gamma} \otimes A_{1}^{\Gamma}}\left(A_{n}^{\Gamma} \otimes\left(A_{1}^{\Gamma} \otimes \bigwedge^{i} V^{\vee}\right)\right) \\
D_{i}:= & A_{n+1}^{\Gamma} \otimes_{A_{n}^{\Gamma} \otimes A_{1}^{\Gamma}}\left(A_{n}^{\Gamma} \otimes\left(A_{1}^{\Gamma} \otimes \wedge^{i} V^{\vee}\right)\right) \\
& \otimes_{A_{n}^{\Gamma}} \bigoplus_{k+l=2}\left(A_{n}^{\Gamma} \otimes\left(\bigwedge^{\vee} \otimes A_{1}^{\Gamma}\right)\right) \otimes_{A_{n}^{\Gamma} \otimes A_{1}^{\Gamma}} A_{n+1}^{\Gamma} \\
& \otimes_{A_{1}^{\Gamma} \otimes A_{n}^{\Gamma}}\left(\left(A_{1}^{\Gamma} \otimes \bigwedge^{\vee}\right) \otimes A_{n}^{\Gamma}\right) .
\end{aligned}
$$

We will prove the case $n=0$, which is the same as the general case but simplifies the notation. In this case, the composition is

$$
\begin{aligned}
& \left(A_{1}^{\Gamma} \otimes \bigwedge^{i} V^{\vee}\right) \\
& \quad \stackrel{f}{\rightarrow}\left(A_{1}^{\Gamma} \otimes \bigwedge^{i} V^{\vee}\right) \otimes_{A_{0}^{\Gamma}}\left(\bigoplus_{k+l=2}\left(\bigwedge^{k} V^{\vee} \otimes A_{1}^{\Gamma}\right) \otimes_{A_{1}^{\Gamma}}\left(A_{1}^{\Gamma} \otimes \bigwedge^{l} V^{\vee}\right)\right)\{2\} \\
& \quad \stackrel{g}{\rightarrow}\left(A_{1}^{\Gamma} \otimes \bigwedge^{i} V^{\vee}\right) .
\end{aligned}
$$

The image of any element under $f$ consists of three terms. The only term that will be nonzero after applying $g$ will be the term

$$
\left(A_{1}^{\Gamma} \otimes \bigwedge^{i} V^{\vee}\right) \otimes_{A_{0}^{\Gamma}}\left(\bigwedge^{2-i} V^{\vee} \otimes A_{1}^{\Gamma}\right) \otimes_{A_{1}^{\Gamma}}\left(A_{1}^{\Gamma} \otimes \bigwedge^{i} V^{\vee}\right)
$$

If $i=0$ and $a \in A_{1}^{\Gamma}$, then

$$
a \mapsto-a \otimes\left(\left(w_{1} \wedge w_{2} \otimes 1\right) \otimes 1\right) \mapsto(a \otimes 1) \cdot \omega\left(w_{1} \wedge w_{2}\right)=a,
$$

which proves that the composition is the identity when $i=0$.

If $i=1$, then

$$
\begin{aligned}
(f, \gamma) \otimes w & \mapsto((f, \gamma) \otimes w) \otimes\left(-\left(w_{1} \otimes 1\right) \otimes\left(1 \otimes w_{2}\right)+\left(w_{2} \otimes 1\right) \otimes\left(1 \otimes w_{1}\right)\right) \\
& \mapsto-\left((f, \gamma) \otimes w_{2}\right) \omega\left(w \wedge w_{1}\right)+\left((f, \gamma) \otimes w_{1}\right) \omega\left(w \wedge w_{2}\right),
\end{aligned}
$$

where $a=(f, \gamma)$. Since all maps are linear in the $w$ 's, it suffices to check the two cases $w=w_{1}$ and $w=w_{2}$. In the first case, the first term is zero and we get

$$
(f, \gamma) \otimes w_{1} \mapsto\left((f, \gamma) \otimes w_{1}\right) \omega\left(w_{1} \wedge w_{2}\right)=(f, \gamma) \otimes w_{1}
$$


while in the second case,

$$
(f, \gamma) \otimes w_{2} \mapsto-\left((f, \gamma) \otimes w_{2}\right) \otimes \omega\left(w_{2} \wedge w_{1}\right)=(f, \gamma) \otimes w_{2} .
$$

If $i=2$ and $a \in A_{1}^{\Gamma}$, then

$$
\begin{aligned}
a \otimes\left(w \wedge w^{\prime}\right) & \mapsto\left(a \otimes\left(w \wedge w^{\prime}\right)\right) \otimes 1 \otimes\left(1 \otimes\left(w_{1} \wedge w_{2}\right)\right) \\
& \mapsto a \otimes\left(w_{1} \wedge w_{2}\right) \omega\left(w \wedge w^{\prime}\right),
\end{aligned}
$$

and it is easy to check that this equals $a \otimes\left(w \wedge w^{\prime}\right)$, so that the composition is the identity.

This concludes the proof of the first relation in (1). The fact that the composition $\mathrm{Q} \stackrel{\operatorname{adj} I}{\longrightarrow} \mathrm{QPQ}[-1]\{1\} \stackrel{I \text { adj }}{\longrightarrow} \mathrm{Q}$ is also the identity follows similarly.

\subsubsection{The composition $\mathrm{P} \stackrel{\text { adj } I}{\longrightarrow} \mathrm{PQP}[1]\{-1\} \stackrel{I \text { adj }}{\longrightarrow} \mathrm{P}$}

This is the same as the composition

$$
\begin{gathered}
A_{n+1}^{\Gamma} \otimes_{A_{n}^{\Gamma} \otimes A_{1}^{\Gamma}}\left(A_{n}^{\Gamma} \otimes \mathbb{k}[\Gamma]\right) \\
\longrightarrow A_{n+1}^{\Gamma} \otimes_{A_{n}^{\Gamma} \otimes A_{1}^{\Gamma}}\left(A_{n}^{\Gamma} \otimes \mathbb{k}[\Gamma] \otimes \mathbb{k}[\Gamma]\right) \otimes_{A_{n}^{\Gamma} \otimes A_{1}^{\Gamma}} A_{n+1}^{\Gamma} \otimes_{A_{n}^{\Gamma} \otimes A_{1}^{\Gamma}}\left(A_{n}^{\Gamma} \otimes \mathbb{k}[\Gamma]\right) \\
\downarrow \\
A_{n+1}^{\Gamma} \otimes_{A_{n}^{\Gamma} \otimes A_{1}^{\Gamma}}\left(A_{n}^{\Gamma} \otimes \mathbb{k}[\Gamma]\right) .
\end{gathered}
$$

Using (21), the first map is given by

$$
1 \otimes(1 \otimes 1) \mapsto \sum_{i=0}^{n} \sum_{\gamma \in \Gamma} s_{i} \cdots s_{n} \otimes\left(1 \otimes \gamma \otimes \gamma^{-1}\right) \otimes s_{n} \cdots s_{i} \otimes(1 \otimes 1) .
$$

Composing with the second map, all terms are zero unless $i=0$ (recall that $s_{i} \cdots s_{n}=$ 1 if $i=0$ ), in which case we get the composition

$$
\begin{aligned}
1 \otimes(1 \otimes 1) & \mapsto \sum_{\gamma \in \Gamma} 1 \otimes\left(1 \otimes \gamma \otimes \gamma^{-1}\right) \otimes 1 \otimes(1 \otimes 1) \\
& \mapsto \sum_{\gamma \in \Gamma} 1 \otimes_{A_{n}^{\Gamma} \otimes A_{1}^{\Gamma}}(1 \otimes \gamma) \otimes_{A_{n}^{\Gamma}}\left(\operatorname{tr}\left(\gamma^{-1}\right)\right) \\
& =1 \otimes_{A_{n}^{\Gamma} \otimes A_{1}^{\Gamma}}(1 \otimes 1) \otimes_{A_{n}^{\Gamma}} \operatorname{tr}(1) \\
& =1 \otimes(1 \otimes 1),
\end{aligned}
$$


where to get the last two equalities we use that $\operatorname{tr}\left(\gamma^{-1}\right)=0$, unless $\gamma=1$, in which case $\operatorname{tr}(1)=1$. This proves that the composition $\mathrm{P} \stackrel{\operatorname{adj} I}{\longrightarrow} \mathrm{PQP}[1] \stackrel{I \text { adj }}{\longrightarrow} \mathrm{P}$ is the identity. The fact that $\mathrm{Q} \stackrel{I \text { adj }}{\longrightarrow} \mathrm{QPQ}[1] \stackrel{\operatorname{adj} I}{\longrightarrow} \mathrm{Q}$ is the identity follows similarly.

\subsection{Dots and adjunctions}

Next we study how natural transformations of $P$ and $Q$ interact with the adjunctions. We will show that the following pairs of maps are equal for any $b \in B^{\Gamma}$ (for simplicity we omit shifts in this section):

(1) $\mathrm{QP} \stackrel{I X(b)}{\longrightarrow} \mathrm{QP} \stackrel{\text { adj }}{\longrightarrow} \mathrm{id}$ and $\mathrm{QP} \stackrel{X(b) I}{\longrightarrow} \mathrm{QP} \stackrel{\text { adj }}{\longrightarrow} \mathrm{id}$;

(2) $\mathrm{PQ} \stackrel{X(b) I}{\longrightarrow} \mathrm{PQ} \stackrel{\text { adj }}{\longrightarrow}$ id and $\mathrm{PQ} \stackrel{I X(b)}{\longrightarrow} \mathrm{PQ} \stackrel{\text { adj }}{\longrightarrow} \mathrm{id}$;

(3) $\quad$ id $\stackrel{\text { adj }}{\longrightarrow} \mathrm{PQ} \stackrel{X(b) I}{\longrightarrow} \mathrm{PQ}$ and id $\stackrel{\text { adj }}{\longrightarrow} \mathrm{PQ} \stackrel{I X(b)}{\longrightarrow} \mathrm{PQ}$;

(4) $\quad$ id $\stackrel{\text { adj }}{\longrightarrow} \mathrm{QP} \stackrel{I X(b)}{\longrightarrow} \mathrm{QP}$ and id $\stackrel{\text { adj }}{\longrightarrow} \mathrm{QP} \stackrel{X(b) I}{\longrightarrow} \mathrm{QP}$.

Equalities (1) and (2) are equivalent to the following defining relations for 2morphisms in $\mathscr{H}_{\Gamma}^{\prime}$ :
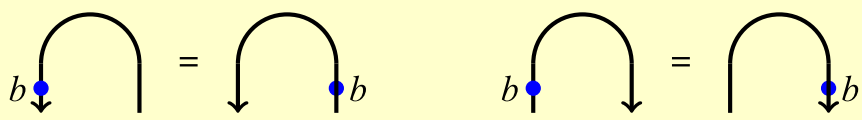

Notice that relations (3) and (4) above follow formally from (1) and (2) via the adjointness properties. Subsequently, we only check (1) and (2). Also, since any $b$ is the composition of $v$ 's and $\gamma$ 's, it suffices to consider the cases when $b=\gamma$ and $b=v$.

\subsubsection{Relation (1) when $b=\gamma$}

Recall that

$$
(n) Q^{\Gamma} \otimes_{A_{n+1}^{\Gamma}} P^{\Gamma}(n)=\left(A_{n}^{\Gamma} \otimes \mathbb{k}[\Gamma]\right) \otimes_{A_{n}^{\Gamma} \otimes A_{1}^{\Gamma}} A_{n+1}^{\Gamma} \otimes_{A_{n}^{\Gamma} \otimes A_{1}^{\Gamma}}\left(A_{n}^{\Gamma} \otimes \mathbb{k}[\Gamma]\right) .
$$

Now the map QP $\stackrel{I X(\gamma)}{\longrightarrow}$ QP in (1) is

$$
\left(1 \otimes \gamma_{1}\right) \otimes a \otimes\left(1 \otimes \gamma_{2}\right) \mapsto\left(1 \otimes \gamma_{1}\right) \otimes a \otimes\left(1 \otimes \gamma_{2} \gamma\right),
$$

where we can assume that $a=1$ or $a=(n, n+1)$. If $a=1$, then QP $\stackrel{\text { adj }}{\longrightarrow}$ id takes this to $\operatorname{tr}\left(\gamma_{1} \gamma_{2} \gamma\right) 1 \in A_{n}^{\Gamma}$. If $a=(n, n+1)$, then this is mapped to zero. On the other hand, QP $\stackrel{X(\gamma) I}{\longrightarrow}$ QP in (1) is

$$
\left(1 \otimes \gamma_{1}\right) \otimes a \otimes\left(1 \otimes \gamma_{2}\right) \mapsto\left(1 \otimes \gamma \gamma_{1}\right) \otimes a \otimes\left(1 \otimes \gamma_{2}\right),
$$

which is then mapped to $\operatorname{tr}\left(\gamma \gamma_{1} \gamma_{2}\right) 1 \in A_{n}^{\Gamma}$ if $a=1$ and to zero if $a=(n, n+1)$. So the two compositions agree. One can prove (2) when $b=\gamma$ similarly. 


\subsubsection{Relation (1) when $b=v$}

To write down the composition QP $\stackrel{I X(v)}{\longrightarrow} \mathrm{QP} \stackrel{\text { adj }}{\longrightarrow}$ id we resolve both copies of $\mathbb{k}[\Gamma]$ in (22). We get a map of complexes which is determined by the composition

$$
C_{1} \stackrel{I \phi_{v} \oplus 0}{\longrightarrow} C_{2} \rightarrow C_{3} \stackrel{\text { adj }}{\longrightarrow} A_{n}^{\Gamma},
$$

where

$$
\begin{aligned}
C_{1}:= & \bigoplus_{k+l=1}\left(A_{n}^{\Gamma} \otimes\left(\bigwedge^{k} V^{\vee} \otimes A_{1}^{\Gamma}\right)\right) \otimes_{A_{n}^{\Gamma} \otimes A_{1}^{\Gamma}} A_{n+1}^{\Gamma} \\
& \otimes_{A_{n}^{\Gamma} \otimes A_{1}^{\Gamma}}\left(A_{n}^{\Gamma} \otimes\left(A_{1}^{\Gamma} \otimes \bigwedge^{l} V^{\vee}\right)\right) \\
C_{2}:= & \left(A_{n}^{\Gamma} \otimes A_{1}^{\Gamma}\right) \otimes_{A_{n}^{\Gamma} \otimes A_{1}^{\Gamma}} A_{n+1}^{\Gamma} \otimes_{A_{n}^{\Gamma} \otimes A_{1}^{\Gamma}}\left(A_{n}^{\Gamma} \otimes A_{1}^{\Gamma}\right) \\
C_{3}:= & \left(A_{n}^{\Gamma} \otimes \mathbb{k}[\Gamma]\right) \otimes_{A_{n}^{\Gamma} \otimes A_{1}^{\Gamma}} A_{n+1}^{\Gamma} \otimes_{A_{n}^{\Gamma} \otimes A_{1}^{\Gamma}}\left(A_{n}^{\Gamma} \otimes \mathbb{k}[\Gamma]\right) .
\end{aligned}
$$

The second map is induced by the natural projection $A_{1}^{\Gamma} \rightarrow \mathbb{k}[\Gamma]$.

If $k=1$, then the first map is zero, and if $k=0$, it is given by

$(1 \otimes a) \otimes a^{\prime} \otimes\left(1 \otimes\left(a^{\prime \prime} \otimes w\right)\right) \mapsto(1 \otimes a) \otimes a^{\prime} \otimes\left(1 \otimes a^{\prime \prime}\langle w, v\rangle\right) \in C_{2}$,

where $a^{\prime} \in A_{n+1}^{\Gamma}$ is either 1 or the transposition $(n, n+1)$. The only case when this term is not mapped to zero is when $a^{\prime}=1$ and both $a, a^{\prime \prime}$ lie in degree 0 . So let $a=\gamma$ and $a^{\prime \prime}=\gamma^{\prime \prime}$, in which case

$$
\operatorname{adj} \circ\left(I \phi_{v}\right)\left((1 \otimes \gamma) \otimes 1 \otimes\left(1 \otimes\left(\gamma^{\prime \prime} \otimes w\right)\right)\right)=1\langle w, v\rangle \operatorname{tr}\left(\gamma \gamma^{\prime \prime}\right) \in A_{n}^{\Gamma} .
$$

Similarly, QP $\stackrel{X(v) I}{\longrightarrow}$ QP $\stackrel{\text { adj }}{\longrightarrow}$ id is determined by the composition $C_{1} \stackrel{0 \oplus \psi_{v} I}{\longrightarrow}$ $C_{2} \rightarrow C_{3} \stackrel{\text { adj }}{\longrightarrow} A_{n}^{\Gamma}$. This is zero if $k=0$. If $k=1$, then the composition is also zero except in terms of the form

$$
\begin{aligned}
(1 \otimes(w \otimes \gamma)) \otimes 1 \otimes\left(1 \otimes \gamma^{\prime \prime}\right) & \mapsto-(1 \otimes\langle v, w\rangle \gamma) \otimes 1 \otimes\left(1 \otimes \gamma^{\prime \prime}\right) \\
& \mapsto-1\langle v, w\rangle \operatorname{tr}\left(\gamma \gamma^{\prime \prime}\right) \in A_{n}^{\Gamma} .
\end{aligned}
$$

Although these two compositions are not equal as maps of complexes, they are homotopic to each other. To see this, we need to define a map $h_{v}$ of $\left(A_{n}^{\Gamma}, A_{n}^{\Gamma}\right)$ bimodules such that the following diagram commutes: 


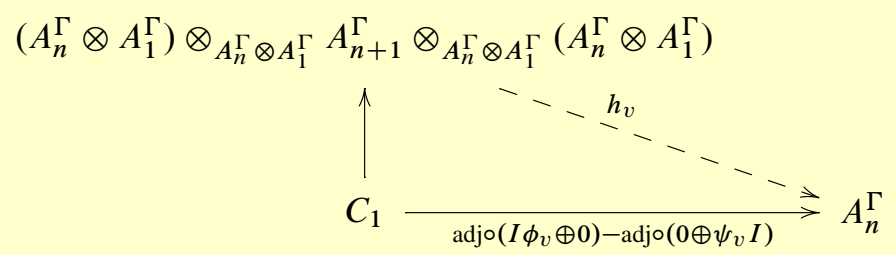

The map $h_{v}$ is determined by where it takes elements of the form

$$
(1 \otimes a) \otimes a^{\prime} \otimes\left(1 \otimes a^{\prime \prime}\right),
$$

where $a, a^{\prime \prime} \in A_{1}^{\Gamma}$ and either $a^{\prime}=1$ or $a^{\prime}=(n, n+1)$. If $a^{\prime}=(n, n+1)$, then $h_{v}$ sends $(1 \otimes a) \otimes a^{\prime} \otimes\left(1 \otimes a^{\prime \prime}\right)$ to zero. If $a^{\prime}=1$, then $h_{v}$ maps it to $\left\langle a a^{\prime \prime}, v\right\rangle 1$, where $\left\langle a a^{\prime \prime}, v\right\rangle$ is zero unless $a a^{\prime \prime}$ is of degree 1 , in which case $\left\langle a a^{\prime \prime}, v\right\rangle=\langle w, v\rangle \operatorname{tr}(\gamma)$ for $a a^{\prime \prime}=(w, \gamma) \in A_{1}^{\Gamma}$.

We now show that diagram (25) commutes. Consider first the term where $k=0$ in $C_{1}$. We see that

$$
\begin{aligned}
C_{1} \ni(1 \otimes \gamma) \otimes 1 \otimes\left(1 \otimes\left(\gamma^{\prime \prime} \otimes w\right)\right) & \mapsto(1 \otimes \gamma) \otimes 1 \otimes\left(1 \otimes\left(\gamma^{\prime \prime} w, \gamma^{\prime \prime}\right)\right) \\
& \stackrel{h_{v}}{\longrightarrow} 1\left\langle\gamma \gamma^{\prime \prime} w, v\right\rangle \operatorname{tr}\left(\gamma \gamma^{\prime \prime}\right) .
\end{aligned}
$$

This composition is zero if $\gamma \gamma^{\prime \prime} \neq 1$ and is equal to $1\langle w, v\rangle \operatorname{tr}(1)$ otherwise.

Now adj $\circ\left(0 \oplus \psi_{v} I\right)$ kills this term, so, using (23), we see this is the same as

$$
\left(\operatorname{adj} \circ\left(I \phi_{v} \oplus 0\right)-\operatorname{adj} \circ\left(0 \oplus \psi_{v} I\right)\right)\left((1 \otimes \gamma) \otimes 1 \otimes\left(1 \otimes\left(\gamma^{\prime \prime} \otimes w\right)\right)\right) .
$$

So diagram (25) commutes when $k=0$.

For the $k=1$ term in $C_{1}$, we have

$C_{1} \ni(1 \otimes(w \otimes \gamma)) \otimes 1 \otimes\left(1 \otimes \gamma^{\prime \prime}\right) \mapsto(1 \otimes(w, \gamma)) \otimes 1 \otimes\left(1 \otimes \gamma^{\prime \prime}\right) \stackrel{h_{v}}{\longrightarrow} 1\langle w, v\rangle \operatorname{tr}\left(\gamma \gamma^{\prime \prime}\right)$.

Also, adj $\circ\left(I \phi_{v} \oplus 0\right)$ acts by zero, and so using (24) we see that

$\left(\operatorname{adj} \circ\left(I \phi_{v} \oplus 0\right)-\operatorname{adj} \circ\left(0 \oplus \psi_{v} I\right)\right)\left((1 \otimes(w \otimes \gamma)) \otimes 1 \otimes\left(1 \otimes \gamma^{\prime \prime}\right)\right)=1\langle v, w\rangle \operatorname{tr}\left(\gamma \gamma^{\prime \prime}\right)$.

So diagram (25) also commutes when $k=1$.

\subsubsection{Relation (2) when $b=v$}

After resolving both copies of $\mathbb{k}[\Gamma]$ in

$$
P^{\Gamma}(n) \otimes_{A_{n}^{\Gamma}}(n) Q^{\Gamma}=A_{n+1}^{\Gamma} \otimes_{A_{n}^{\Gamma} \otimes A_{1}^{\Gamma}}\left(A_{n}^{\Gamma} \otimes \mathbb{k}[\Gamma] \otimes \mathbb{k}[\Gamma]\right) \otimes_{A_{n}^{\Gamma} \otimes A_{1}^{\Gamma}} A_{n+1}^{\Gamma},
$$

the composition $\mathrm{PQ} \stackrel{X(v) I}{\longrightarrow} \mathrm{PQ} \stackrel{\text { adj }}{\longrightarrow}$ id is induced by the map 


$$
\begin{aligned}
& \bigoplus_{k+l=3}\left(A_{1}^{\Gamma} \otimes \bigwedge^{k} V^{\vee}\right) \otimes\left(\bigwedge^{l} V^{\vee} \otimes A_{1}^{\Gamma}\right) \\
& \stackrel{\phi_{v} I \oplus \phi_{v}^{\prime} I}{\longrightarrow} \bigoplus_{k+l=2}\left(A_{1}^{\Gamma} \otimes \bigwedge^{k} V^{\vee}\right) \otimes\left(\bigwedge^{l} V^{\vee} \otimes A_{1}^{\Gamma}\right) \rightarrow A_{1}^{\Gamma}
\end{aligned}
$$

given by

$\left(1 \otimes\left(w \wedge w^{\prime}\right)\right) \otimes\left(w^{\prime \prime} \otimes 1\right) \mapsto\left(-\left\langle w^{\prime}, v\right\rangle 1 \otimes w+\langle w, v\rangle 1 \otimes w^{\prime}\right) \otimes\left(w^{\prime \prime} \otimes 1\right)$

$$
\mapsto 1\left(-\left\langle w^{\prime}, v\right\rangle \omega\left(w \wedge w^{\prime \prime}\right)+\langle w, v\rangle \omega\left(w^{\prime} \wedge w^{\prime \prime}\right)\right)
$$

on one summand and similarly

$$
\begin{aligned}
(1 \otimes w) \otimes\left(\left(w^{\prime} \wedge w^{\prime \prime}\right) \otimes 1\right) & \mapsto 1\langle w, v\rangle \otimes\left(\left(w^{\prime} \wedge w^{\prime \prime}\right) \otimes 1\right) \\
& \mapsto-1\langle w, v\rangle \omega\left(w^{\prime} \wedge w^{\prime \prime}\right)
\end{aligned}
$$

on the second summand.

On the other hand, the composition $\mathrm{PQ} \stackrel{I X(v)}{\longrightarrow} \mathrm{PQ} \stackrel{\text { adj }}{\longrightarrow}$ id is given by

$$
\begin{aligned}
\left(1 \otimes\left(w \wedge w^{\prime}\right)\right) \otimes\left(w^{\prime \prime} \otimes 1\right) & \mapsto\left(1 \otimes\left(w \wedge w^{\prime}\right)\right) \otimes-\left\langle v, w^{\prime \prime}\right\rangle 1 \\
& \mapsto-1\left\langle v, w^{\prime \prime}\right\rangle \omega\left(w \wedge w^{\prime}\right)
\end{aligned}
$$

on one summand and similarly

$(1 \otimes w) \otimes\left(\left(w^{\prime} \wedge w^{\prime \prime}\right) \otimes 1\right) \mapsto(1 \otimes w) \otimes\left(-\left\langle v, w^{\prime}\right\rangle w^{\prime \prime} \otimes 1+\left\langle v, w^{\prime \prime}\right\rangle w^{\prime} \otimes 1\right)$

$$
\mapsto 1\left(-\left\langle v, w^{\prime}\right\rangle \omega\left(w \wedge w^{\prime \prime}\right)+\left\langle v, w^{\prime \prime}\right\rangle \omega\left(w \wedge w^{\prime}\right)\right)
$$

on the other summand. It is now easy to check that these maps are equal. (By the linearity in $w$ it suffices to check the three cases $w=w^{\prime}, w=w^{\prime \prime}$, and $w^{\prime}=w^{\prime \prime}$.)

\subsection{Pitchfork relations}

Next we check that the following compositions are equal:

(1) $\mathrm{P} \stackrel{I \text { adj }}{\longrightarrow} \mathrm{PPQ}[1]\{-1\} \stackrel{T I}{\longrightarrow} \mathrm{PPQ}[1]\{-1\}$ and $\mathrm{P} \stackrel{\operatorname{adj} I}{\longrightarrow} \mathrm{PQP}[1]\{-1\} \stackrel{I T}{\longrightarrow}$ $P P Q[1]\{-1\}$;

$\mathrm{Q} \stackrel{I \text { adj }}{\longrightarrow} \mathrm{QPQ}[1]\{-1\} \stackrel{T I}{\longrightarrow} \mathrm{PQQ}[1]\{-1\}$ and $\mathrm{Q} \stackrel{\operatorname{adj} I}{\longrightarrow} \mathrm{PQQ}[1]\{-1\} \stackrel{I T}{\longrightarrow}$ $P Q Q[1]\{-1\}$;

$$
\begin{aligned}
& \mathrm{PPQ} \underset{I T}{\stackrel{I T}{\longrightarrow}} \mathrm{PQP} \stackrel{\operatorname{adj} I}{\longrightarrow} \mathrm{P}[1]\{-1\} \text { and } \mathrm{PPQ} \stackrel{\text { ad }}{\longrightarrow} \mathrm{PPQ} \underset{T I}{\stackrel{I \text { adj }}{\longrightarrow}} \mathrm{P}[1]\{-1\} ; \\
& \mathrm{PQQ} \stackrel{I T}{\longrightarrow} \mathrm{PQQ} \stackrel{\text { adj }}{\longrightarrow} \mathrm{Q}[1]\{-1\} \text { and } \mathrm{PQQ} \stackrel{T I}{\longrightarrow} \mathrm{QPQ} \stackrel{\text { adj }}{\longrightarrow} \mathrm{Q}[1]\{-1\} .
\end{aligned}
$$


This will check the following defining isotopy relations for 2-morphisms in $\mathscr{H}_{\Gamma}^{\prime}$ :
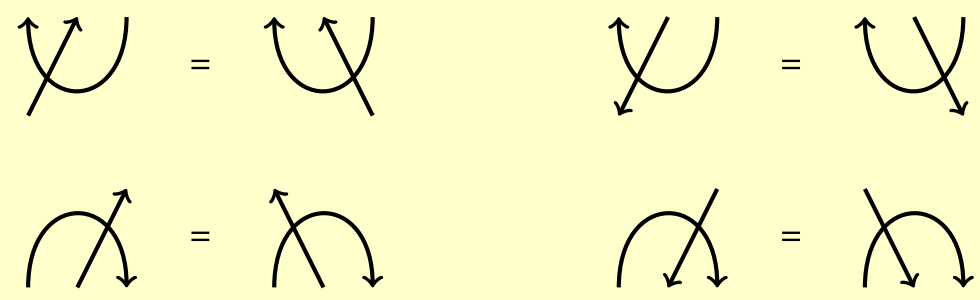

Notice that one also has four more relations where one reverses the orientation of the cups or caps, but these all follow formally from the relations above and the adjointness relations from Section 5.2. These pitchfork relations, together with the adjunction relations which allow the straightening of an S-shape, imply that any two diagrams without dots differing by a rel boundary planar isotopy define the same 2morphism.

\subsubsection{Pitchfork relations (1)}

The first composition is a map,

$$
A_{n+1}^{\Gamma} \otimes_{A_{n}^{\Gamma} \otimes A_{1}^{\Gamma}}\left(A_{n}^{\Gamma} \otimes \mathbb{k}[\Gamma]\right) \stackrel{I \text { adj }}{\longrightarrow} C \stackrel{T I}{\longrightarrow} C,
$$

where

$$
\begin{aligned}
C:= & A_{n+1}^{\Gamma} \otimes_{A_{n}^{\Gamma} \otimes A_{1}^{\Gamma}}\left(A_{n}^{\Gamma} \otimes \mathbb{k}[\Gamma]\right) \otimes_{A_{n}^{\Gamma}} A_{n}^{\Gamma} \otimes_{A_{n-1}^{\Gamma} \otimes A_{1}^{\Gamma}}\left(A_{n-1}^{\Gamma}\right. \\
& \otimes \mathbb{k}[\Gamma] \otimes \mathbb{k}[\Gamma]) \otimes_{A_{n-1}^{\Gamma} \otimes A_{1}^{\Gamma}} A_{n}^{\Gamma} .
\end{aligned}
$$

This composition is determined by

$1 \otimes(1 \otimes 1) \mapsto \sum_{i=0}^{n-1} \sum_{\gamma \in \Gamma} 1 \otimes(1 \otimes 1) \otimes\left(s_{i} \cdots s_{n-1}\right) \otimes\left(1 \otimes \gamma \otimes \gamma^{-1}\right) \otimes\left(s_{n-1} \cdots s_{i}\right)$

$$
\begin{aligned}
& =\sum_{i=0}^{n-1} \sum_{\gamma \in \Gamma}\left(s_{i} \cdots s_{n-1}\right) \otimes(1 \otimes 1) \otimes 1 \otimes\left(1 \otimes \gamma \otimes \gamma^{-1}\right) \otimes\left(s_{n-1} \cdots s_{i}\right) \\
& \mapsto \sum_{i=0}^{n-1} \sum_{\gamma \in \Gamma}\left(s_{i} \cdots s_{n-1} s_{n}\right) \otimes(1 \otimes \gamma) \otimes 1 \otimes\left(1 \otimes 1 \otimes \gamma^{-1}\right) \otimes\left(s_{n-1} \cdots s_{i}\right) .
\end{aligned}
$$

On the other hand, the second composition in (1) is a map,

$$
A_{n+1}^{\Gamma} \otimes_{A_{n}^{\Gamma} \otimes A_{1}^{\Gamma}}\left(A_{n}^{\Gamma} \otimes \mathbb{k}[\Gamma]\right) \stackrel{\operatorname{adj} I}{\longrightarrow} C_{1} \stackrel{I T}{\longrightarrow} C_{2},
$$


where

$$
\begin{aligned}
C_{1}:= & A_{n+1}^{\Gamma} \otimes_{A_{n}^{\Gamma} \otimes A_{1}^{\Gamma}}\left(A_{n}^{\Gamma} \otimes \mathbb{k}[\Gamma] \otimes \mathbb{k}[\Gamma]\right) \otimes_{A_{n}^{\Gamma} \otimes A_{1}^{\Gamma}} A_{n+1}^{\Gamma} \otimes_{A_{n}^{\Gamma} \otimes A_{1}^{\Gamma}}\left(A_{n}^{\Gamma} \otimes \mathbb{k}[\Gamma]\right), \\
C_{2}:= & A_{n+1}^{\Gamma} \otimes_{A_{n}^{\Gamma} \otimes A_{1}^{\Gamma}}\left(A_{n}^{\Gamma} \otimes \mathbb{k}[\Gamma]\right) \otimes_{A_{n}^{\Gamma}} A_{n}^{\Gamma} \\
& \otimes_{A_{n-1}^{\Gamma} \otimes A_{1}^{\Gamma}}\left(A_{n-1}^{\Gamma} \otimes \mathbb{k}[\Gamma] \otimes \mathbb{k}[\Gamma]\right) \otimes_{A_{n-1}^{\Gamma} \otimes A_{1}^{\Gamma}} A_{n}^{\Gamma} .
\end{aligned}
$$

It is determined by

$$
\begin{aligned}
1 \otimes(1 \otimes 1) \mapsto & \sum_{i=0}^{n} \sum_{\gamma \in \Gamma}\left(s_{i} \cdots s_{n}\right) \otimes\left(1 \otimes \gamma \otimes \gamma^{-1}\right) \otimes\left(s_{n} \cdots s_{i}\right) \otimes(1 \otimes 1) \\
= & \sum_{i=1}^{n} \sum_{\gamma \in \Gamma}\left(s_{i} \cdots s_{n}\right) \otimes\left(1 \otimes \gamma \otimes \gamma^{-1}\right) \otimes s_{n} \otimes\left(s_{n-1} \cdots s_{i} \otimes 1\right) \\
& +\sum_{\gamma \in \Gamma} 1 \otimes\left(1 \otimes \gamma \otimes \gamma^{-1}\right) \otimes 1 \otimes(1 \otimes 1) \\
\mapsto & \sum_{i=1}^{n} \sum_{\gamma \in \Gamma}\left(s_{i} \cdots s_{n}\right) \otimes(1 \otimes \gamma) \otimes 1 \otimes\left(1 \otimes 1 \otimes \gamma^{-1}\right) \otimes\left(s_{n-1} \cdots s_{i}\right) .
\end{aligned}
$$

The two compositions are equal, and thus we see that both sides of the first pitchfork relation are equal.

The proof of relation (2) is very similar to the proof of relation (1), and we omit the details.

\subsubsection{Pitchfork relation (3)}

Resolving every copy of $\mathbb{k}[\Gamma]$, the first composition in (3) is

$C_{1}[-1]\{1\} \stackrel{I T}{\longrightarrow} C_{2}[-1]\{1\} \stackrel{\text { adj } I}{\longrightarrow} \bigoplus_{l} A_{n+1}^{\Gamma} \otimes_{A_{n}^{\Gamma} \otimes A_{1}^{\Gamma}}\left(A_{n}^{\Gamma} \otimes\left(A_{1}^{\Gamma} \otimes \bigwedge^{l} V^{\vee}\right)\right)[1]\{-1\}$,

where

$$
\begin{aligned}
C_{1}:= & \bigoplus_{k, l, m} A_{n+1}^{\Gamma} \otimes_{A_{n}^{\Gamma} \otimes A_{1}^{\Gamma}}\left(A_{n}^{\Gamma} \otimes\left(A_{1}^{\Gamma} \otimes \bigwedge^{k} V^{\vee}\right)\right) \otimes_{A_{n}^{\Gamma}} A_{n}^{\Gamma} \\
& \otimes_{A_{n-1}^{\Gamma} \otimes A_{1}^{\Gamma}}\left(A_{n-1}^{\Gamma} \otimes\left(A_{1}^{\Gamma} \otimes \bigwedge^{l} V^{\vee}\right)\right) \\
& \otimes_{A_{n-1}^{\Gamma}}\left(A_{n-1}^{\Gamma} \otimes\left(A_{1}^{\Gamma} \otimes \bigwedge^{m} V^{\vee}\right)\right) \otimes_{A_{n-1}^{\Gamma} \otimes A_{1}^{\Gamma}} A_{n}^{\Gamma},
\end{aligned}
$$




$$
\begin{aligned}
C_{2}:= & \bigoplus_{k, l, m} A_{n+1}^{\Gamma} \otimes_{A_{n}^{\Gamma} \otimes A_{1}^{\Gamma}}\left(A_{n}^{\Gamma} \otimes\left(A_{1}^{\Gamma} \otimes \bigwedge^{k} V^{\vee}\right)\right) \\
& \otimes_{A_{n}^{\Gamma}}\left(A_{n}^{\Gamma} \otimes\left(\bigwedge^{m} V^{\vee} \otimes A_{1}^{\Gamma}\right)\right) \otimes_{A_{n}^{\Gamma} \otimes A_{1}^{\Gamma}} A_{n+1}^{\Gamma} \\
& \otimes_{A_{n}^{\Gamma} \otimes A_{1}^{\Gamma}}\left(A_{n}^{\Gamma} \otimes\left(A_{1}^{\Gamma} \otimes \bigwedge^{l} V^{\vee}\right)\right) .
\end{aligned}
$$

The maps are given by

$1 \otimes(1 \otimes(1 \otimes w)) \otimes 1 \otimes\left(1 \otimes\left(1 \otimes w^{\prime}\right)\right) \otimes\left(1 \otimes\left(1 \otimes w^{\prime \prime}\right)\right) \otimes 1$

$$
\begin{aligned}
& \mapsto(-1)^{l m} 1 \otimes(1 \otimes(1 \otimes w)) \otimes\left(1 \otimes\left(1 \otimes w^{\prime \prime}\right)\right) \otimes s_{n} \otimes\left(1 \otimes\left(1 \otimes w^{\prime}\right)\right) \\
& \mapsto(-1)^{l m} s_{n} \otimes\left(1 \otimes\left(1 \otimes w^{\prime}\right)\right) \omega\left(w \wedge w^{\prime \prime}\right),
\end{aligned}
$$

where $w \in \wedge^{k} V^{\vee}, w^{\prime} \in \wedge^{l} V^{\vee}$, and $w^{\prime \prime} \in \wedge^{m} V^{\vee}$. Here we use the fact that the right crossing $T: \mathrm{PQ} \rightarrow \mathrm{QP}$ acts on the resolutions of $\mathrm{P}$ and $\mathrm{Q}$ just as before, that is, by switching factors and adding a copy of $s_{n}$ in the middle.

On the other hand, the second composition in (3) is

$$
C_{1}[-1]\{1\} \stackrel{T I}{\longrightarrow} C_{1}[-1]\{1\} \stackrel{I \text { adj }}{\longrightarrow} \bigoplus_{l} A_{n+1}^{\Gamma} \otimes_{A_{n}^{\Gamma} \otimes A_{1}^{\Gamma}}\left(A_{n}^{\Gamma} \otimes\left(A_{1}^{\Gamma} \otimes \bigwedge^{l} V^{\vee}\right)\right)[1]\{-1\} .
$$

The maps are given by

$1 \otimes(1 \otimes(1 \otimes w)) \otimes 1 \otimes\left(1 \otimes\left(1 \otimes w^{\prime}\right)\right) \otimes\left(1 \otimes\left(1 \otimes w^{\prime \prime}\right)\right) \otimes 1$

$$
\begin{aligned}
& \mapsto(-1)^{k l} s_{n} \otimes\left(1 \otimes\left(1 \otimes w^{\prime}\right)\right) \otimes 1 \otimes(1 \otimes(1 \otimes w)) \otimes\left(1 \otimes\left(1 \otimes w^{\prime \prime}\right)\right) \otimes 1 \\
& \mapsto(-1)^{k l} s_{n} \otimes\left(1 \otimes\left(1 \otimes w^{\prime}\right)\right) \omega\left(w \wedge w^{\prime \prime}\right) .
\end{aligned}
$$

As long as $k l \equiv l m \bmod 2$, this is the same composition as before. The only other possibility is that $k+m \equiv 1 \bmod 2$, in which case $\omega\left(w \wedge w^{\prime \prime}\right)=0$ (and thus both sides of (3) vanish). Thus relation (3) holds. Relation (4) is proved similarly.

\subsection{Dots and crossings}

Next we check that dots move freely through crossings, namely that the following compositions are equal (for simplicity we omit shifts in this section):

(1) $\mathrm{PP} \stackrel{X(b) I}{\rightarrow} \mathrm{PP} \stackrel{T}{\rightarrow} \mathrm{PP}$ and $\mathrm{PP} \stackrel{T}{\rightarrow} \mathrm{PP} \stackrel{I X(b)}{\rightarrow} \mathrm{PP}$ where $b \in B^{\Gamma}$,

(2) $\quad \mathrm{PP} \stackrel{I X(b)}{\longrightarrow} \mathrm{PP} \stackrel{T}{\rightarrow} \mathrm{PP}$ and $\mathrm{PP} \stackrel{T}{\rightarrow} \mathrm{PP} \stackrel{X(b) I}{\longrightarrow} \mathrm{PP}$ where $b \in B^{\Gamma}$, 

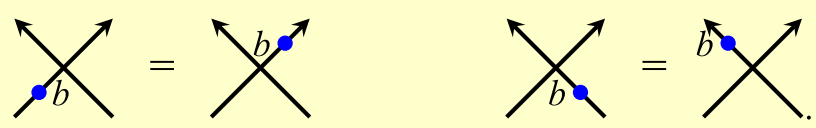

Notice that the analogous relations involving the other three oriented crossings follow formally from the relations above and the adjunctions from the previous sections.

\subsubsection{Relation (1) when $b=\gamma$}

The first composition is $C \stackrel{X(\gamma) I}{\longrightarrow} C \stackrel{T}{\rightarrow} C$, where

$$
C:=A_{n+2}^{\Gamma} \otimes_{A_{n}^{\Gamma} \otimes A_{1}^{\Gamma} \otimes A_{1}^{\Gamma}}\left(A_{n}^{\Gamma} \otimes \mathbb{k}[\Gamma] \otimes \mathbb{k}[\Gamma]\right) .
$$

The map is given by

$$
1 \otimes(1 \otimes 1 \otimes 1) \mapsto 1 \otimes(1 \otimes \gamma \otimes 1) \mapsto s_{n+1} \otimes(1 \otimes 1 \otimes \gamma) .
$$

Similarly, the second composition $C \stackrel{T}{\rightarrow} C \stackrel{I X(\gamma)}{\longrightarrow} C$ is given by

$$
1 \otimes(1 \otimes 1 \otimes 1) \mapsto s_{n+1} \otimes(1 \otimes 1 \otimes 1) \mapsto s_{n+1} \otimes(1 \otimes 1 \otimes \gamma) .
$$

Clearly these two compositions are the same. Relation (2) is proved similarly.

\subsubsection{Relation (2) when $b=v$}

We first replace each $\mathbb{k}[\Gamma]$ by its resolution. Then $T$ lifts to a map of complexes

$A_{n}^{\Gamma} \otimes\left(A_{1}^{\Gamma} \otimes \bigwedge^{k} V^{\vee}\right) \otimes\left(A_{1}^{\Gamma} \otimes \bigwedge^{l} V^{\vee}\right) \rightarrow A_{n}^{\Gamma} \otimes\left(A_{1}^{\Gamma} \otimes \bigwedge^{l} V^{\vee}\right) \otimes\left(A_{1}^{\Gamma} \otimes \bigwedge^{k} V^{\vee}\right)$ given by

$a \otimes\left(a^{\prime} \otimes w^{\prime}\right) \otimes\left(a^{\prime \prime} \otimes w^{\prime \prime}\right) \mapsto(-1)^{k l} a \otimes\left(a^{\prime \prime} \otimes w^{\prime \prime}\right) \otimes\left(a^{\prime} \otimes w^{\prime}\right)$, where $w^{\prime} \in \bigwedge^{k} V^{\vee}$ and $w^{\prime \prime} \in \bigwedge^{l} V^{\vee}$.

Now the composition $T \circ X(v) I$ is induced by $a \otimes\left(a^{\prime} \otimes w^{\prime}\right) \otimes\left(a^{\prime \prime} \otimes w^{\prime \prime}\right) \mapsto a \otimes \phi_{v}\left(a^{\prime} \otimes w^{\prime}\right) \otimes\left(a^{\prime \prime} \otimes w^{\prime \prime}\right)$

$$
\mapsto(-1)^{(k-1) l} a \otimes\left(a^{\prime \prime} \otimes w^{\prime \prime}\right) \otimes \phi_{v}\left(a^{\prime} \otimes w^{\prime}\right),
$$

while $I X(v) \circ T$ is induced by

$$
\begin{aligned}
a \otimes\left(a^{\prime} \otimes w^{\prime}\right) \otimes\left(a^{\prime \prime} \otimes w^{\prime \prime}\right) & \mapsto(-1)^{k l} a \otimes\left(a^{\prime \prime} \otimes w^{\prime \prime}\right) \otimes\left(a^{\prime} \otimes w^{\prime}\right) \\
& \mapsto(-1)^{k l} a \otimes\left(a^{\prime \prime} \otimes w^{\prime \prime}\right) \otimes(-1)^{l} \phi_{v}\left(a^{\prime} \otimes w^{\prime}\right) .
\end{aligned}
$$

These two compositions are clearly equal, which proves relation (1). Relation (2) is proved similarly. 


\subsection{Composition of crossings relations}

First we have the symmetric group relations, namely that the following compositions are equal:

(1) $\mathrm{PP} \stackrel{T}{\rightarrow} \mathrm{PP} \stackrel{T}{\rightarrow} \mathrm{PP}$ and $\mathrm{PP} \stackrel{I I}{\rightarrow} \mathrm{PP}$,

(2) $\mathrm{PPP} \stackrel{I T}{\longrightarrow} \mathrm{PPP} \stackrel{T I}{\longrightarrow} \mathrm{PPP} \stackrel{I T}{\longrightarrow} \mathrm{PPP}$ and $\mathrm{PPP} \stackrel{T I}{\longrightarrow} \mathrm{PPP} \stackrel{I T}{\longrightarrow} \mathrm{PPP} \stackrel{T I}{\longrightarrow} \mathrm{PPP}$,
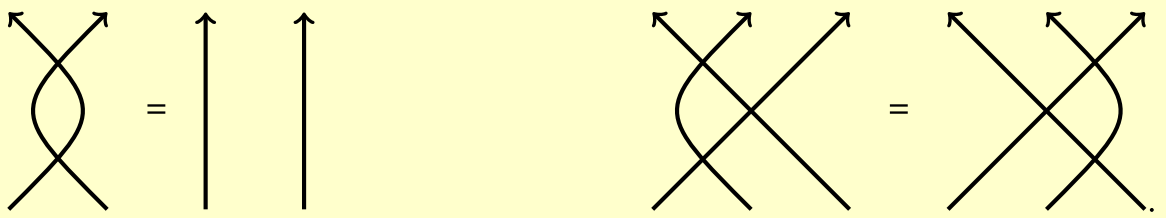

These follow immediately from the definition of $T$ on upward-pointing strands, since the action of $T$ is given by multiplication by a simple reflection in the symmetric group.

On the other hand, we also have the following relations involving oppositely pointing strands (for simplicity we omit shifts in the rest of this section):

(1) $\mathrm{PQ} \stackrel{I I}{\rightarrow} \mathrm{PQ}$ and $\mathrm{PQ} \stackrel{T}{\rightarrow} \mathrm{QP} \stackrel{T}{\rightarrow} \mathrm{PQ}$,

(2) the identity $\mathrm{QP} \stackrel{I I}{\rightarrow} \mathrm{QP}$ can be decomposed as the composition $\mathrm{QP} \stackrel{T}{\rightarrow} \mathrm{PQ} \stackrel{T}{\rightarrow}$ $\mathrm{QP}$ plus the sum over a basis of elements $b \in B^{\Gamma}$ of the composition $\mathrm{QP} \stackrel{I X(b)}{\longrightarrow} \mathrm{QP} \stackrel{\text { adj }}{\longrightarrow} \mathrm{id} \stackrel{\text { adj }}{\longrightarrow} \mathrm{QP} \stackrel{I X\left(b^{\vee}\right)}{\longrightarrow} \mathrm{QP}$,
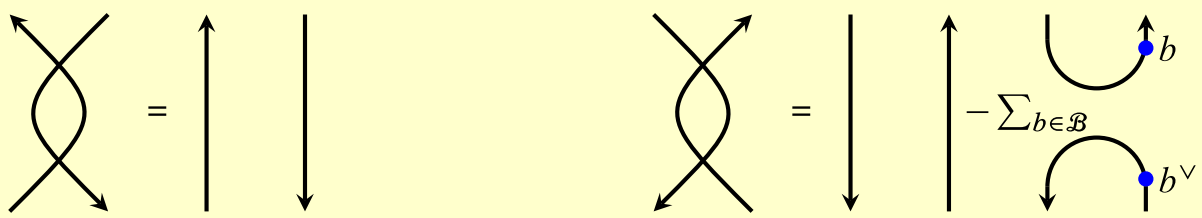

Relation (1) is straightforward to check. The first composition is $C_{1} \stackrel{T}{\rightarrow} C_{2} \stackrel{T}{\rightarrow} C_{1}$, where

$$
\begin{aligned}
& C_{1}:=A_{n}^{\Gamma} \otimes_{A_{n-1}^{\Gamma} \otimes A_{1}^{\Gamma}}\left(A_{n-1}^{\Gamma} \otimes \mathbb{k}[\Gamma] \otimes \mathbb{k}[\Gamma]\right) \otimes_{A_{n-1}^{\Gamma} \otimes A_{1}^{\Gamma}} A_{n}^{\Gamma}, \\
& C_{2}:=\left(A_{n}^{\Gamma} \otimes \mathbb{k}[\Gamma]\right) \otimes_{A_{n}^{\Gamma} \otimes A_{1}^{\Gamma}} A_{n+1}^{\Gamma} \otimes_{A_{n}^{\Gamma} \otimes A_{1}^{\Gamma}}\left(A_{n}^{\Gamma} \otimes \mathbb{k}[\Gamma]\right) .
\end{aligned}
$$

This composition is given by

$$
1 \otimes(1 \otimes 1 \otimes 1) \otimes 1 \mapsto(1 \otimes 1) \otimes s_{n} \otimes(1 \otimes 1) \mapsto 1 \otimes(1 \otimes 1 \otimes 1) \otimes 1
$$

and is thus equal to the identity.

Relation (2) is more interesting. The composition QP is given by the $\left(A_{n}^{\Gamma}, A_{n}^{\Gamma}\right)$ bimodule 


$$
\left(A_{n}^{\Gamma} \otimes \mathbb{k}[\Gamma]\right) \otimes_{A_{n}^{\Gamma} \otimes A_{1}^{\Gamma}} A_{n+1}^{\Gamma} \otimes_{A_{n}^{\Gamma} \otimes A_{1}^{\Gamma}}\left(A_{n}^{\Gamma} \otimes \mathbb{k}[\Gamma]\right) .
$$

Since the tensor products are derived, we need to resolve one copy of $\mathbb{k}[\Gamma]$. We resolve the second copy of $\mathbb{k}[\Gamma]$. We end with a complex where the terms are

$$
\bigoplus_{k=0}^{2}\left(A_{n}^{\Gamma} \otimes \mathbb{k}[\Gamma]\right) \otimes_{A_{n}^{\Gamma} \otimes A_{1}^{\Gamma}} A_{n+1}^{\Gamma} \otimes_{A_{n}^{\Gamma} \otimes A_{1}^{\Gamma}}\left(A_{n}^{\Gamma} \otimes\left(A_{1}^{\Gamma} \otimes \bigwedge^{k} V^{\vee}\right)\right)[k] .
$$

Now, up to the $\left(A_{n}^{\Gamma}, A_{n}^{\Gamma}\right)$-action, any element is of the form

$$
\left(1 \otimes \gamma^{\prime}\right) \otimes 1 \otimes(1 \otimes(1 \otimes w)) \quad \text { or } \quad\left(1 \otimes \gamma^{\prime}\right) \otimes s_{n} \otimes(1 \otimes(1 \otimes w)),
$$

where $w \in \wedge^{k} V^{\vee}$.

If it is an element of the first form, then the differential becomes zero and the map $T: \mathrm{QP} \rightarrow \mathrm{PQ}$ maps it to zero. On the other hand, suppose that $w=w_{1}$, then the composition

$$
\mathrm{QP} \stackrel{I X(b)}{\longrightarrow} \mathrm{QP} \stackrel{\text { adj }}{\longrightarrow} \mathrm{id} \stackrel{\text { adj }}{\longrightarrow} \mathrm{QP} \stackrel{I X\left(b^{\vee}\right)}{\longrightarrow} \mathrm{QP},
$$

where $b=(v, \gamma) \in B^{\Gamma}$, is zero unless $v \in V$, in which case we get

$$
\begin{aligned}
\left(1 \otimes \gamma^{\prime}\right) \otimes 1 \otimes\left(1 \otimes\left(1 \otimes w_{1}\right)\right) \\
\quad \stackrel{I X(v)}{\longrightarrow}\left(1 \otimes \gamma^{\prime}\right) \otimes 1 \otimes\left(1 \otimes\left(1\left\langle v, w_{1}\right\rangle\right)\right) \\
\quad \stackrel{I X(\gamma)}{\longrightarrow}\left(1 \otimes \gamma^{\prime}\right) \otimes 1 \otimes\left(1 \otimes\left(\gamma\left\langle v, w_{1}\right\rangle\right)\right) \\
\quad \stackrel{\text { adj }}{\longrightarrow} \operatorname{tr}\left(\gamma^{\prime} \gamma\right)\left\langle v, w_{1}\right\rangle \\
\quad \stackrel{\text { adj }}{\longrightarrow} \operatorname{tr}\left(\gamma^{\prime} \gamma\right)\left\langle v, w_{1}\right\rangle(1 \otimes 1) \otimes 1 \otimes\left(1 \otimes\left(1 \otimes w_{1} \wedge w_{2}\right)\right) \\
\stackrel{I X\left(\gamma^{-1}\right)}{\longrightarrow} \operatorname{tr}\left(\gamma^{\prime} \gamma\right)\left\langle v, w_{1}\right\rangle(1 \otimes 1) \otimes 1 \otimes\left(1 \otimes\left(\gamma^{-1} \otimes w_{1} \wedge w_{2}\right)\right) \\
\stackrel{I X\left(v^{\vee}\right)}{\longrightarrow} \operatorname{tr}\left(\gamma^{\prime} \gamma\right)\left\langle v, w_{1}\right\rangle(1 \otimes 1) \otimes 1 \\
\quad \otimes\left(1 \otimes\left(\gamma^{-1} \otimes w_{1}\left\langle w_{2}, v^{\vee}\right\rangle-\gamma^{-1} \otimes w_{2}\left\langle w_{1}, v^{\vee}\right\rangle\right)\right) .
\end{aligned}
$$

Now we sum over all $\gamma \in \Gamma$ and over $v=v_{1}, v_{2}$. Since $\left\langle w_{i}, v_{j}\right\rangle=\delta_{i j}$ and $\operatorname{tr}\left(\gamma^{\prime} \gamma\right)=0$, unless $\gamma^{\prime}=\gamma^{-1}$ we get

$$
\begin{gathered}
\left\langle v_{1}, w_{1}\right\rangle(1 \otimes 1) \otimes 1 \otimes\left(1 \otimes\left(\gamma^{\prime} \otimes w_{1}\left\langle w_{2}, v_{2}\right\rangle-\gamma^{\prime} \otimes w_{2}\left\langle w_{1}, v_{2}\right\rangle\right)\right) \\
=\left(1 \otimes \gamma^{\prime}\right) \otimes 1 \otimes\left(1 \otimes\left(1 \otimes w_{1}\right)\right) .
\end{gathered}
$$

Thus the composition acts by the identity if $w=w_{1}$ and similarly if $w=w_{2}$. Likewise, one can show that it also acts by the identity if $w=1$ or $w=w_{1} \wedge w_{2}$. 
If we are dealing with an element of the second form, then it is in the image of the differential unless $k=0$. So we can assume that $k=0$, in which case we need to consider

$$
(1 \otimes \gamma) \otimes s_{n} \otimes(1 \otimes 1) \in\left(A_{n}^{\Gamma} \otimes \mathbb{k}[\Gamma]\right) \otimes_{A_{n}^{\Gamma} \otimes A_{1}^{\Gamma}} A_{n+1}^{\Gamma} \otimes_{A_{n}^{\Gamma} \otimes A_{1}^{\Gamma}}\left(A_{n}^{\Gamma} \otimes A_{1}^{\Gamma}\right) .
$$

The composition involving cups and caps maps it to zero (because the cap takes it to zero). Meanwhile, the composition $\mathrm{QP} \stackrel{T}{\rightarrow} \mathrm{PQ} \stackrel{T}{\rightarrow} \mathrm{PQ}$ maps it as follows:

$$
\begin{aligned}
& (1 \otimes \gamma) \otimes s_{n} \otimes(1 \otimes 1) \mapsto 1 \otimes(1 \otimes 1 \otimes \gamma) \otimes 1 \mapsto(1 \otimes 1) \otimes s_{n} \otimes(\gamma \otimes 1) \\
& =(1 \otimes \gamma) \otimes s_{n} \otimes(1 \otimes 1) .
\end{aligned}
$$

Thus in both cases we see that the sum of the two compositions takes the element to itself. This proves relation (2).

\subsection{Counterclockwise circles and curls}

Finally, we show that

(1) the composition $\mathrm{P} \stackrel{\operatorname{adj} I}{\longrightarrow} \mathrm{QPP}[-1]\{1\} \stackrel{I T}{\longrightarrow} \mathrm{QPP}[-1]\{1\} \stackrel{\operatorname{adj} I}{\longrightarrow} \mathrm{P}[-2]\{2\}$ is zero, and

(2) the composition

$$
\mathrm{id} \stackrel{\text { adj }}{\longrightarrow} \mathrm{QP}[-1]\{1\} \stackrel{I X(b)}{\longrightarrow} \mathrm{QP}[-1+|b|]\{1-|b|\} \stackrel{\text { adj }}{\longrightarrow} \mathrm{id}[-2+|b|]\{2-|b|\}
$$

is equal to id $\stackrel{\operatorname{tr}(b) I}{\longrightarrow}$ id for any $b \in B^{\Gamma}$ :
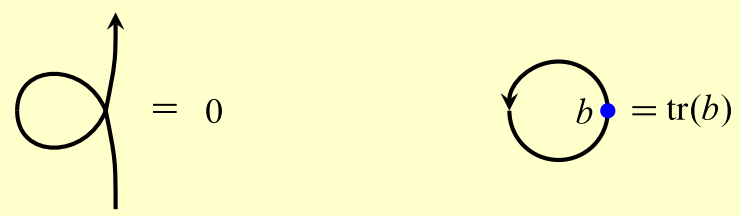

The first composition is straightforward and follows for the same reason that the composition id $\rightarrow \mathrm{QP}[-1]\{1\} \rightarrow \mathrm{id}[-2]\{2\}$ is zero. Alternatively, the left-twist curl is a map $\mathrm{P} \rightarrow \mathrm{P}[-2]\{2\}$, and every such map is zero by degree considerations.

For the second composition we just need to check the case $b=v_{1} \wedge v_{2}$ (see Section 3.5). In that case, one may note that working $\mathbb{k}^{\times}$-equivariantly the only degree 0 maps id $\rightarrow$ id are given by scalars. The result follows by the remark in the first part of Section 3.5.

Alternatively, one can prove the second relation above directly. We must show that the composition 


$$
\mathrm{id} \stackrel{\text { adj }}{\longrightarrow} \mathrm{QP}[-1]\{1\} \stackrel{I X\left(v_{1}\right)}{\longrightarrow} \mathrm{QP} \stackrel{I X\left(v_{2}\right)}{\longrightarrow} \mathrm{QP}[1]\{-1\} \stackrel{\text { adj }}{\longrightarrow} \mathrm{id}
$$

is equal to the identity.

The first map is given by

$$
\begin{aligned}
1 \mapsto & (1 \otimes 1) \otimes 1 \otimes\left(\left(1 \otimes w_{1} \wedge w_{2}\right) \otimes 1\right)-\left(1 \otimes\left(w_{1} \otimes 1\right)\right) \otimes 1 \otimes\left(\left(1 \otimes w_{2}\right) \otimes 1\right) \\
& +\left(1 \otimes\left(w_{2} \otimes 1\right)\right) \otimes 1 \otimes\left(\left(1 \otimes w_{1}\right) \otimes 1\right)-\left(1 \otimes\left(w_{1} \wedge w_{2} \otimes 1\right)\right) \otimes 1 \otimes(1 \otimes 1),
\end{aligned}
$$

where the right-hand terms lie in $\bigoplus_{k+l=2}\left(A_{n}^{\Gamma} \otimes\left(\bigwedge^{k} V^{\vee} \otimes A_{1}^{\Gamma}\right)\right) \otimes_{A_{n}^{\Gamma} \otimes A_{1}^{\Gamma}}$ $A_{n+1}^{\Gamma} \otimes_{A_{1}^{\Gamma} \otimes A_{n}^{\Gamma}}\left(\left(A_{1}^{\Gamma} \otimes \bigwedge^{l} V^{\vee}\right) \otimes A_{n}^{\Gamma}\right)$. The only term that survives after acting by $I X\left(v_{1}\right)$ and $I X\left(v_{2}\right)$ is the first one, which is then mapped

$$
\begin{aligned}
(1 \otimes 1) \otimes 1 \otimes\left(\left(1 \otimes w_{1} \wedge w_{2}\right) \otimes 1\right) \\
\quad \stackrel{I X\left(v_{1}\right)}{\longrightarrow}(1 \otimes 1) \otimes 1 \otimes\left(\left(\left\langle w_{1}, v_{1}\right\rangle 1 \otimes w_{2}-\left\langle w_{2}, v_{1}\right\rangle 1 \otimes w_{2}\right) \otimes 1\right) \\
\quad \stackrel{I X\left(v_{2}\right)}{\longrightarrow}(1 \otimes 1) \otimes 1 \otimes\left(\left\langle w_{2}, v_{2}\right\rangle\left\langle w_{1}, v_{1}\right\rangle 1 \otimes 1\right) \\
\quad \stackrel{\text { adj }}{\longrightarrow} \operatorname{tr}(1) 1=1 .
\end{aligned}
$$

Here we have used the fact that $\left\langle w_{i}, v_{j}\right\rangle=\delta_{i j}$.

\section{The alternate 2-category $\mathscr{H}^{\Gamma}$}

In order to describe the structure of the 2-category $\mathscr{H}_{\Gamma}$, it is convenient to introduce yet another 2-category $\mathscr{H}^{\Gamma}$. The 2-categories $\mathscr{H}^{\Gamma}$ and $\mathscr{H}_{\Gamma}$ are not equivalent, but are rather "Morita equivalent." This means that they have the same 0-morphisms and 1morphisms and that for any 1-morphism $A$ the algebras $\operatorname{End}_{\mathscr{H}_{\Gamma}}(A)$ and $\operatorname{End}_{\mathscr{H} \Gamma}(A)$ are Morita equivalent. This should mean that the 2-representation theories of $\mathscr{H}^{\Gamma}$ and $\mathscr{H}_{\Gamma}$ are equivalent.

To be more precise, recall that if $\Gamma$ is a finite group, then $\mathbb{k}[\Gamma]$ is Morita equivalent (though not necessarily isomorphic) to a basic algebra $B(\Gamma)$, which is a direct sum of several copies of $\mathbb{k}$, one copy for each irreducible representation of $\Gamma$. It is sometimes convenient to replace $\mathbb{k}[\Gamma]$ with $B(\Gamma)$, whose representation theory is the same.

In our case, if $\Gamma$ is of type $\mathrm{A}$, then $\mathbb{k}[\Gamma] \cong B(\Gamma)$ and hence $\mathcal{H}_{\Gamma}$ and $\mathscr{H}^{\Gamma}$ are actually isomorphic. If $\Gamma$ is not of type $A$, then $\mathbb{k}[\Gamma]$ will contain blocks of dimension greater than one and then $\mathbb{k}[\Gamma] \not B(\Gamma)$. To go from $\mathscr{H}_{\Gamma}$ to $\mathscr{H}^{\Gamma}$ we simply replace $\mathbb{k}[\Gamma]$ by $B(\Gamma)$ in our constructions. Thus $\mathscr{H}^{\Gamma}$ will be Morita equivalent to $\mathscr{H}_{\Gamma}$, but its graphical calculus for 2-morphisms is easier to describe and visualize because there are fewer idempotents. Moreover, there is a canonical isomorphism of algebras $K_{0}\left(\mathscr{H}^{\Gamma}\right) \cong K_{0}\left(\mathscr{H}_{\Gamma}\right)$ (see Proposition 4) and so $\mathscr{H}^{\Gamma}$ is also a categorification of $\mathfrak{h}_{\Gamma}$, which is of independent interest. 


\subsection{The 2-category $\mathscr{H}^{\Gamma}$}

We now give an explicit description of $\mathscr{H}^{\Gamma}$. Fix an orientation $\epsilon$ of the Dynkin diagram. If vertices $i$ and $j$ are connected by an edge in the diagram, we set $\epsilon_{i j}=1$ if the oriented edge with tail $i$ and head $j$ agrees with the orientation $\epsilon$, and $\epsilon_{i j}=-1$ if the oriented edge with tail $i$ and head $j$ disagrees with $\epsilon$. We also set $\epsilon_{i j}=0$ if $i$ and $j$ are not connected by an edge. Thus $\epsilon_{i j}=-\epsilon_{j i}$.

We define an additive $\mathbb{k}$-linear 2-category $\mathscr{H}^{\prime \Gamma}$ as follows. As before, the objects of $\mathscr{H}^{\prime \Gamma}$ are indexed by the integers $\mathbb{Z}$. The 1 -morphisms are generated by objects $P_{i}$ and $Q_{i}$, one for each $i \in I_{\Gamma}$. Thus a 1-morphism of $\mathscr{H}^{\prime \Gamma}$ is a finite composition (sequence) of $P_{i}$ 's and $Q_{j}$ 's.

The space of 2-morphisms between two 1-morphisms is the $\mathbb{k}$-algebra generated by suitable planar diagrams modulo local relations. The diagrams consist of oriented compact 1-manifolds (possibly carrying dots and crossings) immersed into the plane strip $\mathbb{R} \times[0,1]$ modulo isotopies fixing the boundary. Each such strand is labeled by some $i \in I_{\Gamma}$.

Corresponding to the edge in the Dynkin diagram connecting vertex $i$ to vertex $j$ there is a 2-morphism $X: P_{i} \rightarrow P_{j}\langle 1\rangle$ denoted by a solid dot:

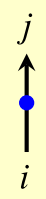

These dots are allowed to move freely along the 1-manifold, including along the cups and caps and through crossings. However, as was the case with degree 1 dots in $\mathscr{H}_{\Gamma}^{\prime}$, dots between strands labeled by distinct nodes $i$ and $j$ (which have degree 1) on faraway strands anticommute when they move past one another.

For each $i \in I_{\Gamma}$ we also include as a defining 2-morphism a solid dot on a strand whose endpoints are both labeled $i$,

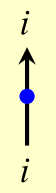

and we denote this map $X: P_{i} \rightarrow P_{i}\langle 2\rangle$. These $i i$ dots also move freely on strands. There is a relation which governs the composition of dots: 


$$
\left.j\}_{i}^{k}=\delta_{i k} \epsilon_{i j}\right\}_{i}^{i}
$$

Next, we have the following relations analogous to relations (9)-(11). For all $i, j, k \in I_{\Gamma}$ we have
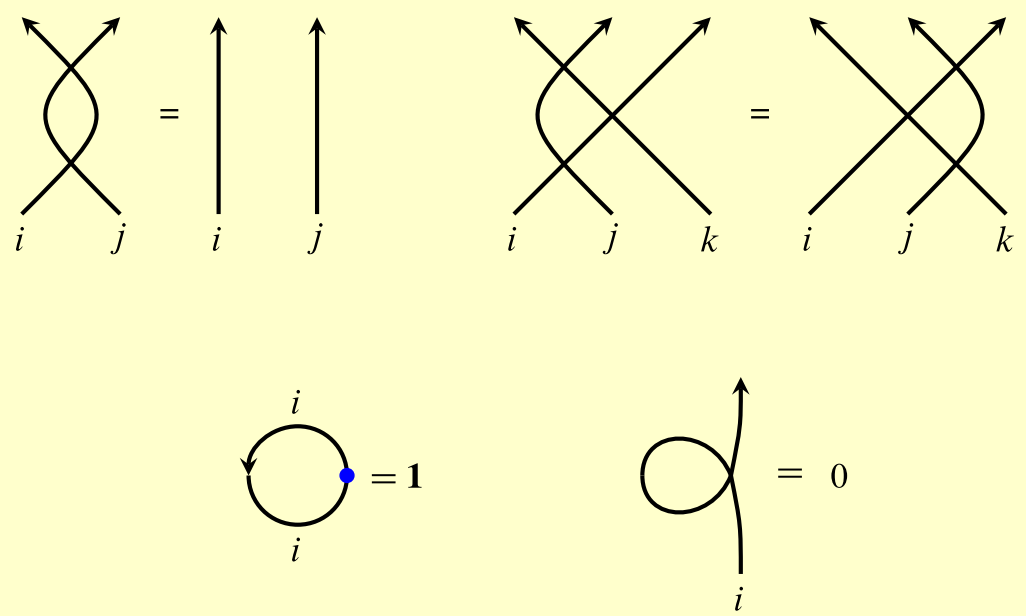

If $i \neq j$, then

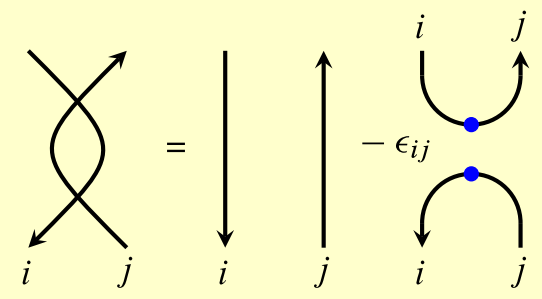

while

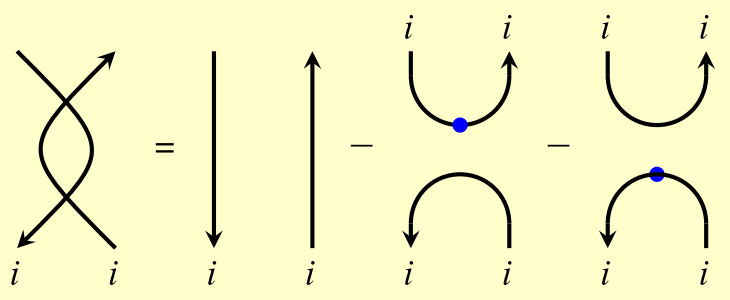


We assign a $\mathbb{Z}$-grading on the space of planar diagrams by defining
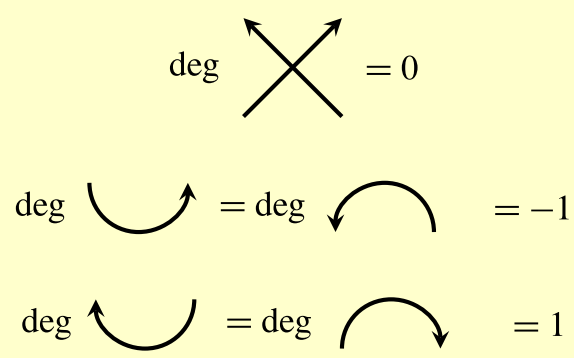

We define the degree of an $i j$ dot to be one (if $i \neq j$ are joined by an edge) and consequently the degree of an $i i$ dot to be two. Equipped with these assignments all the graphical relations are graded. This provides a $\mathbb{Z}$-grading on $\mathscr{H}^{\prime \Gamma}$.

Define $\mathscr{H}^{\Gamma}$ to be the Karoubi envelope of $\mathscr{H}^{\prime \Gamma}$. Since $\mathbb{k}\left[S_{n}\right] \subset \operatorname{End}\left(P_{i}^{n}\right)$ we let $P_{i}^{\lambda}:=\left(P_{i}^{n}, e_{\lambda}\right)$, where $e_{\lambda}$ is the minimal idempotent of $\mathbb{k}\left[S_{n}\right]$ associated to the partition $\lambda$ of $n$. We define $Q_{i}^{\lambda}$ similarly.

\subsection{The functor $\eta: \mathscr{H}^{\Gamma} \longrightarrow \mathscr{H}_{\Gamma}$}

We will define a functor $\mathscr{H}^{\prime \Gamma} \longrightarrow \mathscr{H}_{\Gamma}$. (This induces the functor $\eta: \mathscr{H}^{\Gamma} \rightarrow \mathscr{H}_{\Gamma}$ since $\mathscr{H}_{\Gamma}$ is idempotent complete.)

To define this functor we map $P_{i}$ to $\left(P, e_{i, 1}\right)$ and by adjunction $Q_{i}$ to $\left(Q, e_{i, 1}\right)$. Now the 2-morphism $X: P_{i} \rightarrow P_{i}\langle 2\rangle$ is mapped to the 2-morphism

$$
\frac{|\Gamma|}{\operatorname{dim}\left(V_{i}\right)} e_{i, 1} \cdot \omega \cdot e_{i, 1}:\left(P, e_{i, 1}\right) \rightarrow\left(P, e_{i, 1}\right)\langle 2\rangle .
$$

(The factor $\frac{|\Gamma|}{\operatorname{dim}\left(V_{i}\right)}$ is there to ensure that the left relation in (28) holds.)

Now suppose that $i, j \in I_{\Gamma}$ are joined by an oriented edge $i \rightarrow j$. Then $\operatorname{Hom}_{\mathcal{H}_{\Gamma}}\left(\left(P, e_{i, 1}\right),\left(P, e_{j, 1}\right)\right)$ is one-dimensional, spanned by some map $\alpha_{i j}$. So we map the dot $X: P_{i} \rightarrow P_{j}\langle 1\rangle$ to some multiple of $\alpha_{i j}$. To determine this multiple consider the composition

$$
\alpha_{j i} \circ \alpha_{i j}:\left(P, e_{i, 1}\right) \rightarrow\left(P, e_{i, 1}\right)\langle 2\rangle,
$$

which is nonzero because of the structure of $B^{\Gamma}$. Since $\operatorname{Hom}_{\mathscr{H}_{\Gamma}}\left(\left(P, e_{i, 1}\right),\left(P, e_{i, 1}\right)\right)$ is spanned by $e_{i, 1} \cdot \omega \cdot e_{i, 1}$, this means that $\alpha_{j i} \circ \alpha_{i j}$ equals $e_{i, 1} \cdot \omega \cdot e_{i, 1}$ up to some nonzero multiple. We rescale $\alpha_{i j}$ so that $\alpha_{j i} \circ \alpha_{i j}=\frac{|\Gamma|}{\operatorname{dim}\left(V_{i}\right)} e_{i, 1} \cdot \omega \cdot e_{i, 1}$.

Finally, we map crossings $T: P_{i} P_{j} \rightarrow P_{j} P_{i}$ to crossings $T:\left(P, e_{i, 1}\right)(P$, $\left.e_{j, 1}\right) \rightarrow\left(P, e_{j, 1}\right)\left(P, e_{i, 1}\right)$.

PROPOSITION 3

The above morphisms define a functor $\eta: \mathscr{H}^{\Gamma} \longrightarrow \mathscr{H}_{\Gamma}$. 


\section{Proof}

We need to check relations (26)-(30). Relations (27) and the right-hand relation in (28) are clear. The left-hand relation in (28) holds since

$$
\operatorname{tr}\left(\omega \cdot e_{i, 1}\right)=\frac{\operatorname{dim}\left(V_{i}\right)}{|\Gamma|}
$$

and we mapped $X: P_{i} \rightarrow P_{i}\langle 2\rangle$ to $\frac{|\Gamma|}{\operatorname{dim}\left(V_{i}\right)} e_{i, 1} \cdot \omega \cdot e_{i, 1}$.

Relation (26) holds by definition if $\epsilon_{i j}=1$. If $\epsilon_{i j}=-1$, then $\epsilon_{j i}=1$, and so we have

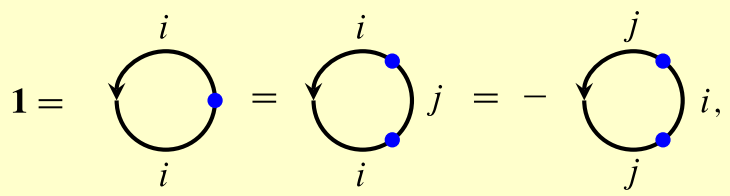

where we use relation (26) to obtain the second equality and the minus sign appears when one passes the two degree 1 dots past each other. It follows that

$$
\alpha_{i j} \circ \alpha_{j i}=-\alpha_{j i} \circ \alpha_{i j}=-\frac{|\Gamma|}{\operatorname{dim}\left(V_{i}\right)} e_{i, 1} \cdot \omega \cdot e_{i, 1},
$$

which proves relation (26) when $\epsilon_{i j}=-1$.

Next we prove relation (30). This follows from the left relation in (10) in the definition of $\mathscr{H}_{\Gamma}^{\prime}$. More precisely, multiply endpoints of all strands of the left relation in (10) by the idempotent $e_{i, 1}$. Now $e_{i, 1} b e_{i, 1}=0$ unless $b$ has degree 0 or 2 . Now if $b$ has degree 0 , then it belongs to $\mathbb{k}[\Gamma]$. We use the basis of $\mathbb{k}[\Gamma]$ consisting of matrix units $b$ which are zero everywhere except in one entry. Then $e_{i, 1} b e_{i, 1}=0$ unless $b=e_{i, 1}$. Thus the sum in (10) collapses and we get only one term corresponding to $b=e_{i, 1}$.

Similarly, if $b$ has degree 2, then the sum collapses and we get the term $b=$ $\left(\omega, e_{i, 1}\right)$. Now

$$
e_{i, 1}^{\vee}=\frac{|\Gamma|}{\operatorname{dim}\left(V_{i}\right)} e_{i, 1} \cdot \omega \cdot e_{i, 1}
$$

since $\operatorname{tr}\left(\omega \cdot e_{i, 1}\right)=\frac{\operatorname{dim}\left(V_{i}\right)}{|\Gamma|}$. This is precisely the image of $X: P_{i} \rightarrow P_{i}\langle 2\rangle$ under $\eta$. Thus we get relation (30).

Finally, relation (29) is similar. In this case only one of the terms in the sum in (10) survives. To compute the coefficient $-\epsilon_{i j}$, cap off everything with a cap containing an $i j$ dot and use relations (26) and (28).

We will prove in Section 7 that $\eta$ induces an isomorphism on Grothendieck groups. 
PROPOSITION 4

The functor $\eta$ induces an isomorphism:

$$
K_{0}(\eta): K_{0}\left(\mathscr{H}^{\Gamma}\right) \longrightarrow K_{0}\left(\mathscr{H}_{\Gamma}\right)
$$

Thus both $\mathscr{H}^{\Gamma}$ and $\mathscr{H}_{\Gamma}$ categorify the Heisenberg algebra $\mathfrak{h}_{\Gamma}$.

\subsection{Proof of Proposition 2}

In this section we prove that relations from Proposition 2 hold in $\mathscr{H}^{\Gamma}$. Since we have a functor $\eta: \mathscr{H}^{\Gamma} \rightarrow \mathscr{H}_{\Gamma}$ which sends $P_{i}$ to $P_{i}$ and $Q_{i}$ to $Q_{i}$, these relations also hold in $\mathscr{H}_{\Gamma}$.

The idempotent $e_{\text {triv }}=\frac{1}{n !} \sum_{g \in S_{n}} g$ corresponding to the trivial representation in $\mathbb{k}\left[S_{n}\right]$ defines the summand $P_{i}^{(n)}=\left(P_{i}^{n}, e_{\text {triv }}\right)$ of $P_{i}^{n}$ in the category $\mathscr{H}^{\Gamma}$. These idempotents also define $Q_{i}^{(n)}$ by adjunction. We will draw $e_{\text {triv }}$ as a white rectangle labeled by $i^{n}$. We will also write a single line connecting two such rectangles instead of $n$ lines, for simplicity. Thus the identity 2-morphism of $P_{i}^{(n)}$ is drawn as one of the pictures

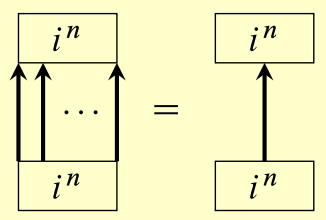

We first prove that

$$
P_{i}^{(n)} P_{j}^{(m)} \cong P_{j}^{(m)} P_{i}^{(n)} \quad \text { for all } i, j \in I_{\Gamma} .
$$

The isomorphism is given by the 2-morphism

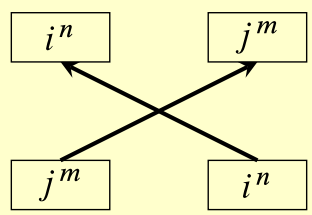

with the inverse given by

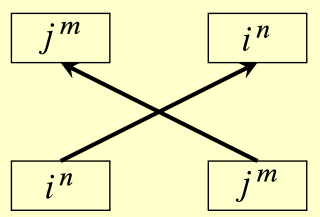


Since we can pull strands apart using the left-hand relation in (27), it is easy to see that these two 2-morphisms are inverse of each other. The proof that

$$
Q_{i}^{(n)} Q_{j}^{(m)} \cong Q_{j}^{(m)} Q_{i}^{(n)} \quad \text { for all } i, j \in I_{\Gamma}
$$

is the same, except that all strands now point downward. The relation $Q_{i}^{(n)} P_{j}^{(m)} \cong$ $P_{j}^{(m)} Q_{i}^{(m)}$ when $\langle i, j\rangle=-1$ follows via the same computation using relation (29).

Next we show the more interesting relation

$$
Q_{i}^{(n)} P_{i}^{(m)} \cong \bigoplus_{k \geq 0} P_{i}^{(m-k)} Q_{i}^{(n-k)} \otimes H^{\star}\left(\mathbb{P}^{k}\right) \quad \text { for all } i \in I_{\Gamma} .
$$

To define maps $Q_{i}^{(n)} P_{i}^{(m)} \rightarrow P_{i}^{(m-k)} Q_{i}^{(n-k)}$ consider the 2-morphisms

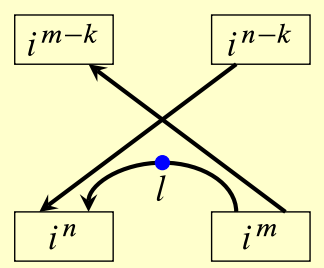

where $0 \leq l \leq k$. In this picture, $k$ of the strands coming out from the bottom are involved in the $k$ caps, while the other strands are involved in crossings. The $l$ tells us that we put a single $i i$ dot on the first $l$ of the $k$ caps. (Because we have the idempotents it does not matter on which $l$ strands we place the dots.)

Taking the direct sum as $l$ ranges from 0 to $k$ defines a 2-morphism from

$$
Q_{i}^{(n)} P_{i}^{(m)} \rightarrow P_{i}^{(m-k)} Q_{i}^{(n-k)} \otimes H^{\star}\left(\mathbb{P}^{k}\right)
$$

Taking the direct sum over $k$ we obtain a 2-morphism:

$$
f: Q_{i}^{(n)} P_{i}^{(m)} \longrightarrow \bigoplus_{k \geq 0} P_{i}^{(m-k)} Q_{i}^{(n-k)} \otimes H^{\star}\left(\mathbb{P}^{k}\right) .
$$

We claim that this morphism is invertible, and we explicitly construct its inverse as a linear combination of diagrams of the form

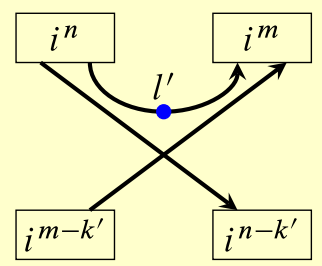


where we put $l^{\prime}$ dots on $l^{\prime}$ of the $k^{\prime}$ cup-like strands.

To do this we first compute the following graphical relation in $\operatorname{End}\left(Q_{i}^{(n)} P_{i}^{(m)}\right)$ :

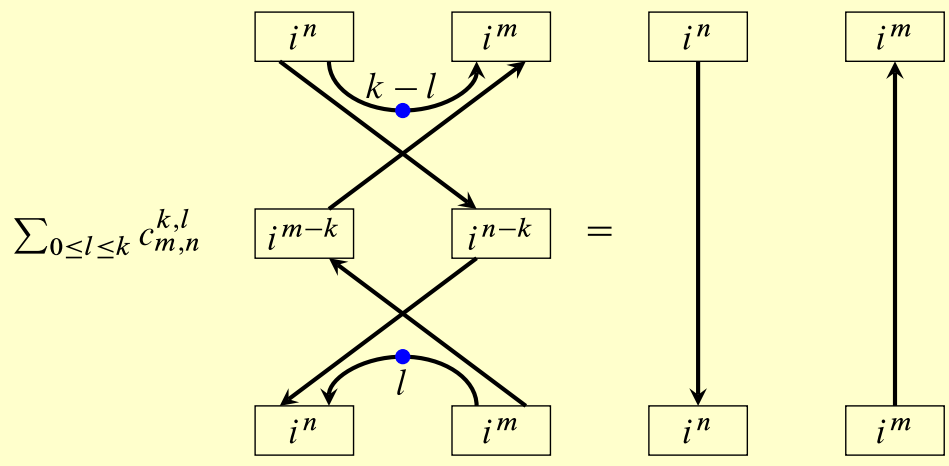

where $c_{m, n}^{k, l}=k !\left(\begin{array}{c}m \\ k\end{array}\right)\left(\begin{array}{l}n \\ k\end{array}\right)\left(\begin{array}{l}k \\ l\end{array}\right)$.

To see where this relation comes from consider the $k=0$ term in the left-hand side. (It has no cups, caps, or dots in it.) Expanding the idempotents $i^{n}$ and $i^{m}$ explicitly as a sum of permutations, we rewrite the $k=0$ term as a linear combination of diagrams with many crossings. Then crossings involving two upwardpointing strands or two downward-pointing strands can be absorbed into the idempotents at the top or bottom of the diagram. (Notice that this is a consequence of using the idempotent $e_{\text {triv }}$, which satisfies $\sigma e_{\text {triv }}=e_{\text {triv }} \sigma=e_{\text {triv }}$ for any permutation $\sigma$.) Thus we write the $k=0$ term as a diagram with no idempotents in the middle:

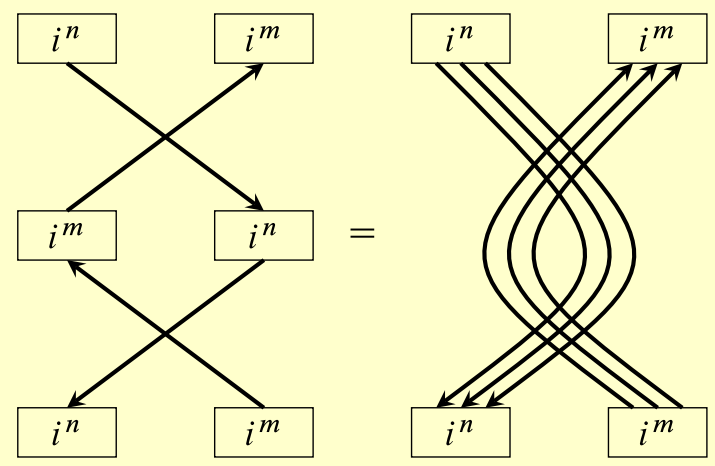

In both sides of the above picture there are $n$ strands emanating from an idempotent $i^{n}$, and in the right-hand side we have drawn more than one of these strands to emphasize the fact that there are many crossings.

More generally, we use the relation 

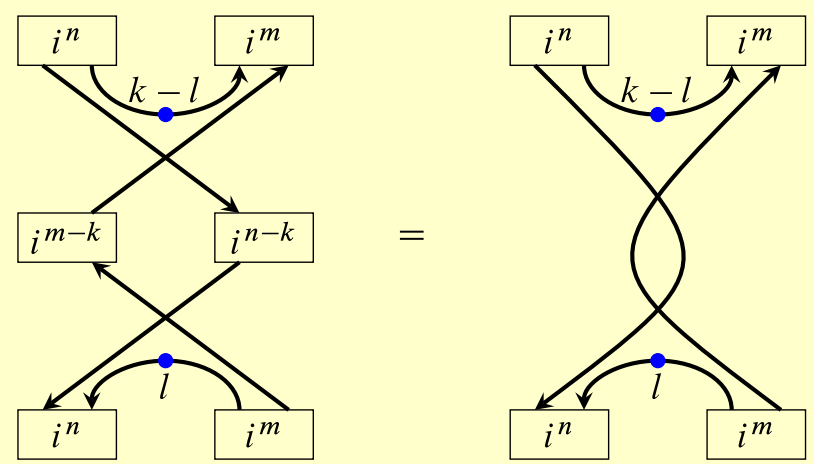

to write the entire left-hand side of equation (31) as a linear combination of diagrams without up-up or down-down crossings.

Now we start pulling apart the inner double crossings in each term one at a time using relation (30). At every stage we absorb newly created upward- (or downward-) pointing crossings into the top (or bottom) idempotents. The result is a linear combination of diagrams of the form

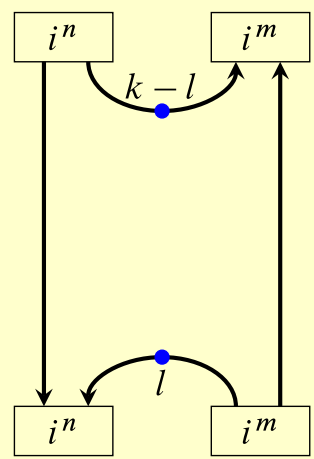

Keeping track of the constants carefully one arrives at equation (31). The particular values of the constants $c_{m, n}^{k, l}$ are not really important apart from the fact that they are nonzero. The key observation which follows from equation (31) is that the identity 2-morphism of $Q_{i}^{(n)} P_{i}^{(m)}$ factors through $P_{i}^{(m-k)} Q_{i}^{(n-k)} \otimes H^{\star}\left(\mathbb{P}^{k}\right)$ as a composition of 2-morphisms

$$
\mathrm{id}: Q_{i}^{(n)} P_{i}^{(m)} \stackrel{f}{\rightarrow} P_{i}^{(m-k)} Q_{i}^{(n-k)} \otimes H^{\star}\left(\mathbb{P}^{k}\right) \stackrel{g}{\rightarrow} Q_{i}^{(n)} P_{i}^{(m)},
$$

where 


$$
f=\sum_{0 \leq l \leq k}
$$

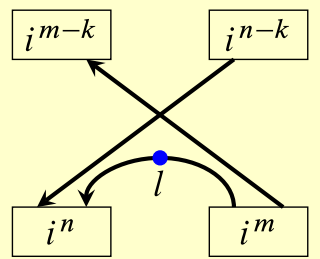

and

$$
g=\sum_{0 \leq l \leq k} c_{m, n}^{k, l}
$$

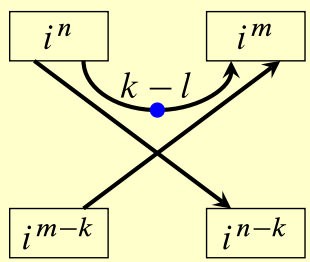

In addition, a similar graphical manipulation shows that
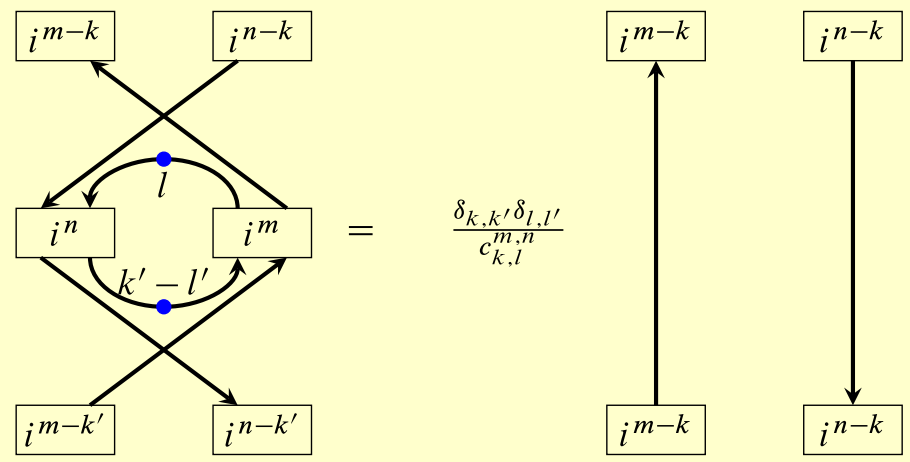

The fact that the left-hand side is zero when $l \neq l^{\prime}$ follows from the fact that when the middle idempotents are expanded as a linear combination of permutations, each term contains either a left-twist curl (which is zero) or a strand with two $i i$ dots on it (which is also zero).

It follows from equation (32) that the identity 2-morphism of $\bigoplus_{k \geq 0} P_{i}^{(m-k)} \times$ $Q_{i}^{(n-k)} \otimes H^{\star}\left(\mathbb{P}^{k}\right)$ factors as

$\mathrm{id}: \bigoplus_{k \geq 0} P_{i}^{(m-k)} Q_{i}^{(n-k)} \otimes H^{\star}\left(\mathbb{P}^{k}\right) \stackrel{g}{\rightarrow} Q_{i}^{(n)} P_{i}^{(m)} \stackrel{f}{\rightarrow} \bigoplus_{k \geq 0} P_{i}^{(m-k)} Q_{i}^{(n-k)} \otimes H^{\star}\left(\mathbb{P}^{k}\right)$,

while the identity 2-morphism of $Q_{i}^{(n)} P_{i}^{(m)}$ factors as $f \circ g$. Thus $f$ and $g$ are inverse isomorphisms, as desired. 
The proof that $Q_{j}^{(n)} P_{i}^{(m)} \cong P_{i}^{(m)} Q_{j}^{(n)} \oplus P_{i}^{(m-1)} Q_{j}^{(n-1)}$ when $\langle i, j\rangle=-1$ is similar to the proof above. In particular, the isomorphism $f^{\prime}$ from the left-hand side to the right-hand side is given by

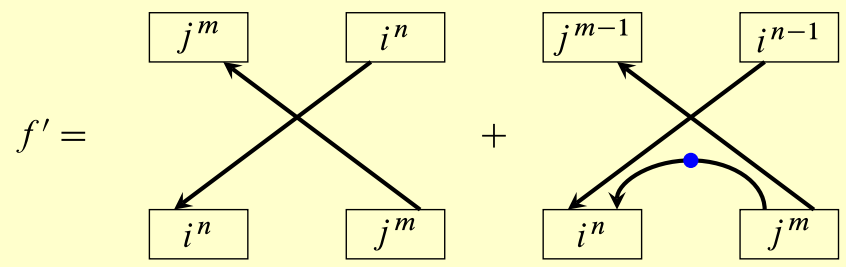

Note that there are no terms of the form

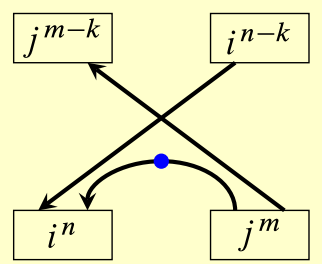

for $k>1$. (This is in contrast to the isomorphism in the corresponding relation for $i=j$.) This difference between the $i=j$ and $\langle i, j\rangle=-1$ case comes from the fact that $i j$ dots anticommute. This anticommutation of dots implies that the above diagram is equal to zero when $k>1$ : grow a crossing between two adjacent strands from the $j^{m}$ symmetrizer, move the two degree 1 dots on those strands past each other (picking up a sign), and then absorb the crossing back into the bottom symmetrizer:

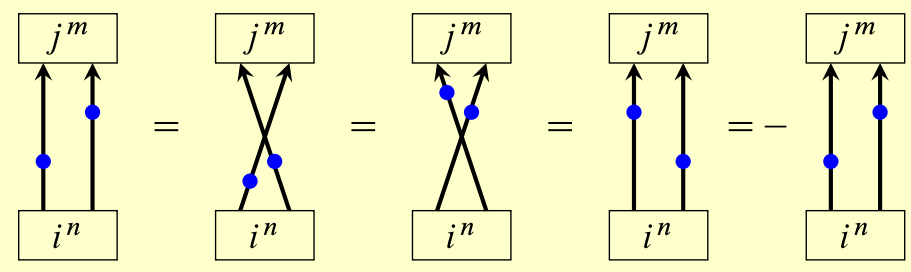

Thus one cannot have more than one dot connecting the idempotents $i^{n}$ and $j^{m}$.

\subsection{Further relations among 1-morphisms}

\section{PROPOSITION 5}

For $i, j \in I_{\Gamma}$ we have isomorphisms in $\mathscr{H}^{\Gamma}$ :

(1) $P_{i}^{(m)}$ and $P_{j}^{\left(1^{n}\right)}$ commute while $Q_{i}^{(m)}$ and $Q_{j}^{\left(1^{n}\right)}$ also commute;
$Q_{i}^{\left(1^{n}\right)} P_{i}^{(m)} \cong P_{i}^{(m)} Q_{i}^{\left(1^{n}\right)}$
$\oplus P_{i}^{(m-1)} Q_{i}^{\left(1^{n-1}\right)}$
$\otimes_{\mathbb{k}} H^{\star}\left(\mathbb{P}^{1}\right) \oplus P_{i}^{(m-2)} Q_{i}^{\left(1^{n-2}\right)} ;$ 


$$
\begin{aligned}
& Q_{j}^{\left(1^{n}\right)} P_{i}^{(m)} \cong \bigoplus_{k \geq 0} P_{i}^{(m-k)} Q_{j}^{\left(1^{n-k}\right)} \text { if }\langle i, j\rangle=-1 ; \\
& Q_{j}^{\left(1^{n}\right)} P_{i}^{(m)} \cong P_{i}^{(m)} Q_{j}^{\left(1^{n}\right)} \text { if }\langle i, j\rangle=0 .
\end{aligned}
$$

The graphical proofs of this statement are analogous to, and no more difficult than, the proofs of Section 6.3; we have omitted the details in the interest of space. We do point out, however, the fact that there are many summands on the right-hand side of the relations

$$
Q_{j}^{\left(1^{n}\right)} P_{i}^{(m)} \cong \bigoplus_{k \geq 0} P_{i}^{(m-k)} Q_{j}^{\left(1^{n-k}\right)}, \quad\langle i, j\rangle=-1,
$$

in contrast to the decomposition of $Q_{j}^{(n)} P_{i}^{(m)}$ (which has only two summands). This is because a trivial idempotent $e_{\text {triv }, i}$ and a sign idempotent labeled $e_{\text {sign, } j}$ can have more than one dot between them, and thus there are nontrivial 2-morphisms from $P_{i}^{(m-k)} Q_{j}^{\left(1^{n-k}\right)}$ to $Q_{j}^{\left(1^{n}\right)} P_{i}^{(m)}$. In particular, the diagrammatic computation analogous to that of equation (33) does not show anything when one of the idempotents is a sign idempotent and the other is a trivial idempotent; since $s_{i} e_{\text {sign }}=e_{\text {sign }} s_{i}=-e_{\text {sign }}$, absorbing a crossing into the sign idempotent introduces $\mathrm{a}-1$.

\subsection{The functor $\Psi$}

We define a covariant autoequivalence $\Psi^{\prime}: \mathscr{H}^{\prime \Gamma} \longrightarrow \mathscr{H}^{\prime \Gamma}$ as follows. $\Psi^{\prime}$ is the identity on objects and on 1-morphisms and is also the identity on cups, caps, and dots. On the other hand, $\Psi^{\prime}$ acts as multiplication by -1 on any crossing between two adjacent strands. This map induces a covariant autoequivalence $\Psi$ on $\mathscr{H}^{\Gamma}$ whose square is the identity.

Since a crossing of two upward-pointing strands is multiplied by -1 , it follows that $\Psi$ takes the idempotent 2-morphism $e_{\text {triv }}$ to the idempotent 2-morphism $e_{\text {sign }}$ (and vice versa). Thus

$$
\begin{aligned}
\Psi\left(P_{i}^{(n)}\right) & =P_{i}^{\left(1^{n}\right)}, \quad \Psi\left(P_{i}^{\left(1^{n}\right)}\right)=P_{i}^{(n)}, \quad \Psi\left(Q_{i}^{(n)}\right)=Q_{i}^{\left(1^{n}\right)}, \\
\Psi\left(Q_{i}^{\left(1^{n}\right)}\right) & =Q_{i}^{(n)} .
\end{aligned}
$$

It follows from the existence of the automorphism $\Psi$ that decompositions between products of $P_{i}^{\left(1^{n}\right)}$, s and $Q_{i}^{\left(1^{n}\right)}$, s have the same form as decompositions between products of $P_{i}^{(n)}$, s and $Q_{i}^{(n)}$, s. The autoequivalence $\Psi$ descends in the Grothendieck group to the automorphism $\psi$ of Section 2.2.3.

\section{Proof of Theorem 1}

In this section we study the sequence of maps 


$$
\mathfrak{h}_{\Gamma} \stackrel{\pi}{\rightarrow} K_{0}\left(\mathscr{H}^{\Gamma}\right) \stackrel{K_{0}(\eta)}{\longrightarrow} K_{0}\left(\mathscr{H}_{\Gamma}\right) \rightarrow \operatorname{End}\left(\bigoplus_{n \geq 0} K_{0}\left(\varphi_{n}^{\Gamma}\right)\right) \simeq \operatorname{End}(\mathcal{F}),
$$

where $\mathcal{F}$ is isomorphic to the Fock space representation of $\mathfrak{h}_{\Gamma}$. We will show that both $\pi$ and $K_{0}(\eta)$ are isomorphisms, thus proving Proposition 4 and Theorem 1. In particular, this shows that both $\mathscr{H}_{\Gamma}$ and $\mathscr{H}^{\Gamma}$ categorify $\mathfrak{h}_{\Gamma}$.

We begin with some preliminary results.

\section{LEMMA 4}

There are no negative-degree endomorphisms of $\prod_{i} P_{i}^{m_{i}} \prod_{i} Q_{i}^{n_{i}}$, while the algebra of degree 0 endomorphisms is isomorphic to $\bigotimes_{i} \mathbb{k}\left[S_{m_{i}}\right] \otimes_{i} \mathbb{k}\left[S_{n_{i}}\right]$.

\section{Proof}

Any negative-degree endomorphism must contain a left-oriented cap or right-oriented cup (since these are the only generating 2-morphisms of negative degree). Such a cup or cap is either part of a left-twist curl (which makes the entire picture equal to zero) or part of a counterclockwise circle. However, by the defining relations, the only nonzero counterclockwise circles are those of nonnegative degree (i.e., those carrying dots of degree 2). Thus any negative-degree endomorphism must be zero.

Note that this argument would not apply to endomorphisms of a 1-morphism that contains a $P$ to the right of a $Q$. For example, a left-oriented cap followed by a right-oriented cap is a nonzero degree -2 endomorphism of $Q_{i} P_{i}$.

By the same argument, any degree 0 diagram describing an endomorphism of $\prod_{i} P_{i}^{m_{i}} \prod_{i} Q_{i}^{n_{i}}$ cannot have any right-twist curls or dots thus must be isotopic to a sum of braid-like diagrams (i.e., diagrams with no local minima or maxima and no dots) connecting a $P_{i}$ to a $P_{i}$ or a $Q_{i}$ to $Q_{i}$. The result now follows.

\section{PROPOSITION 6}

Any 1-morphism in $\mathscr{H}^{\Gamma}$ decomposes uniquely into a finite direct sum of indecomposables. The indecomposable 1-morphisms in $\mathscr{H}^{\Gamma}$ are of the form $\prod_{i} P_{i}^{\lambda_{i}} \prod_{i} Q_{i}^{\mu_{i}}$ for some partitions $\left\{\lambda_{i}, \mu_{i}\right\}$.

\section{Proof}

Lemma 4 implies that the space of endomorphisms (of a fixed degree) of any 1morphism is finite-dimensional. This means that $\mathscr{H}^{\Gamma}$ satisfies the Krull-Schmidt property, and hence any 1-morphism decomposes uniquely into a finite direct sum of indecomposables.

Now any 1-morphism in $\mathscr{H}^{\Gamma}$ is a direct summand of a sequence of $P_{i}$ 's and $Q_{i}$ 's. Using relations from Proposition 1 (which were shown to hold for $\mathscr{H}^{\Gamma}$ in Section 6.3), 
it follows that any 1-morphism is a direct sum of (shifts of) 1-morphisms of the form $\prod_{i} P_{i}^{\lambda_{i}} \prod_{i} Q_{i}^{\mu_{i}}$. It remains to show that such endomorphisms are indecomposable.

But this follows from Lemma 4 since $\prod_{i} P_{i}^{\lambda_{i}} \prod_{i} Q_{i}^{\mu_{i}}$ is a direct summand of $\prod_{i} P_{i}^{\left|\lambda_{i}\right|} \prod_{i} Q_{i}^{\left|\mu_{i}\right|}$ corresponding to the minimal idempotent $\prod_{i} e_{\lambda_{i}, 1} \prod_{i} e_{\mu_{i}, 1} \in$ $\bigotimes_{i} \mathbb{k}\left[S_{\left|\lambda_{i}\right|}\right] \bigotimes_{i} \mathbb{k}\left[S_{\left|\mu_{i}\right|}\right]$.

\section{Remark 5}

Arguing as above, it is straightforward to check that the 1-morphisms $P^{\lambda} Q^{\mu}$ for all $I_{\Gamma}$-colored partitions $\lambda$ and $\mu$ form a complete set of indecomposable 1-morphisms in $\mathscr{H}_{\Gamma}$. The classes of these indecomposable 1-morphisms in the Grothendieck group thus form a "canonical basis" of the Heisenberg algebra. This basis is analogous in many respects to the canonical bases of Kashiwara and Lusztig in the representation theory of quantum groups.

\section{LEMMA 5}

In $\mathscr{H}^{\Gamma}$ we have $P_{i}^{\lambda} \cdot P_{i}^{\mu} \cong \bigotimes_{v}\left(P_{i}^{\nu}\right)^{\oplus c_{\lambda, \mu}^{v}}$, where $c_{\lambda, \mu}^{\nu}$ is the Littlewood-Richardson coefficient. In particular, we have

$$
P_{i}^{(n)} P_{i}^{(m)} \cong \bigoplus_{k=0}^{\min (n, m)} P_{i}^{(n+m-k, k)} \quad \text { and } \quad P_{i}^{(m)} P_{j}^{\left(1^{n}\right)} \cong P_{i}^{\left(m, 1^{n}\right)} \oplus P_{i}^{\left(m+1,1^{n-1}\right)} .
$$

\section{Proof}

The statement follows from the representation theory of symmetric groups. Let $V_{\lambda}$ and $V_{\mu}$ be irreducible representations of $S_{n}$ and $S_{m}$, respectively. Then $V_{\lambda} \otimes V_{\mu}$ is an irreducible representation of $S_{n} \times S_{m}$. This representation, when induced to $S_{n+m}$ decomposes as a direct sum of irreducible representations $V_{\nu}$, and the multiplicity of $V_{\nu}$ in this decomposition is the Littlewood-Richardson coefficient $c_{\lambda, \mu}^{v}$.

This fact about representations of symmetric groups can be rephrased completely in terms of idempotents. If $e_{\lambda} \in \mathbb{k}\left[S_{n}\right], e_{\mu} \in \mathbb{k}\left[S_{m}\right]$, and $e_{\nu} \in \mathbb{k}\left[S_{n+m}\right]$ are idempotents such that $V_{\lambda} \cong \mathbb{k}\left[S_{n}\right] e_{\lambda}, V_{\mu} \cong \mathbb{k}\left[S_{m}\right] e_{\mu}$, and $V_{\nu} \cong \mathbb{k}\left[S_{m+n}\right] e_{\nu}$, then we have

$$
\mathbb{k}\left[S_{n+m}\right] e_{\lambda} e_{\mu} \cong \bigoplus_{s} \mathbb{k}\left[S_{n+m}\right] e_{s}
$$

for some minimal idempotents $\left\{e_{s}\right\}$ in $\mathbb{k}\left[S_{n+m}\right]$. The number of such $e_{s}$ such that $\mathbb{k}\left[S_{n+m}\right] e_{s} \cong \mathbb{k}\left[S_{n+m}\right] e_{\nu}$ is the Littlewood-Richardson coefficient $c_{\lambda, \mu}^{v}$. Under the embeddings

$$
\mathbb{k}\left[S_{n}\right] \otimes_{\mathbb{k}} \mathbb{k}\left[S_{m}\right] \subset \mathbb{k}\left[S_{n+m}\right] \hookrightarrow \operatorname{End}_{\mathscr{H} \Gamma}\left(P_{i}^{n+m}\right),
$$

this decomposition of idempotents implies the decomposition in the lemma. 


\section{COROLLARY 1}

The classes $\left\{\left[P_{i}^{(m)}\right],\left[Q_{j}^{(n)}\right]: i, j \in I_{\Gamma}, n, m \geq 0\right\}$ generate $K_{0}\left(\mathscr{H}^{\Gamma}\right)$ as an algebra.

\section{Proof}

By Proposition $6, K_{0}\left(\mathscr{H}^{\Gamma}\right)$ is spanned by elements of the form $\prod_{i}\left[P_{i}^{\lambda_{i}}\right] \prod_{i}\left[Q_{i}^{\mu_{i}}\right]$. Thus it suffices to show that any $\left[P_{i}^{\lambda}\right]$ is generated by $\left\{\left[P_{i}^{(m)}\right]: m \geq 0\right\}$. This follows by induction using Lemma 5. For instance,

$$
P_{i}^{2}=P_{i}^{(2)} \oplus P_{i}^{\left(1^{2}\right)}
$$

so that $P_{i}^{\left(1^{2}\right)}$ is generated by $P_{i}$ and $P_{i}^{(2)}$.

\section{Remark 6}

The expression of $\left[P_{i}^{\lambda}\right]$ in terms of $\left[P_{i}^{(m)}\right]$ 's is given by the Giambelli identity expressing the Schur polynomial in terms of products of elementary symmetric functions. Explicitly,

$$
\left[P_{i}^{\lambda}\right]=\operatorname{det}_{k l}\left[P_{i}^{\left(\lambda_{k}^{\prime}+k-l\right)}\right]
$$

where $\lambda^{\prime}=\left(\lambda_{1}^{\prime} \geq \lambda_{2}^{\prime} \geq \cdots\right)$ is the transpose of the partition $\lambda$.

In order to prove Proposition 4 and Theorem 1, we now consider the sequence

$$
\mathfrak{h}_{\Gamma} \stackrel{\pi}{\rightarrow} K_{0}\left(\mathscr{H}^{\Gamma}\right) \stackrel{K_{0}(\eta)}{\longrightarrow} K_{0}\left(\mathscr{H}_{\Gamma}\right) \rightarrow \operatorname{End}\left(\bigoplus_{n \geq 0} K_{0}\left(\mathcal{C}_{n}^{\Gamma}\right)\right) \simeq \operatorname{End}(\mathcal{F})
$$

and show that both $\pi$ and $K_{0}(\eta)$ are isomorphisms.

First we check that $\pi$ is injective. This follows since the Fock space representation of $\mathfrak{h}_{\Gamma}$ is faithful, which in turn follows easily from its description as differential operators acting on a polynomial algebra (see [9], [10]). Thus the composition of all maps in the above sequence is injective, which implies that $\pi$ is injective.

Next we check that $\pi$ is surjective. Note that by Lemma $1, K_{0}\left(\mathscr{H}^{\Gamma}\right)$ is generated as an algebra by the classes $\left[P_{i}^{(m)}\right]$ and $\left[Q_{j}^{(n)}\right]$. Since $\pi\left(p_{i}^{(m)}\right)=\left[P_{i}^{(m)}\right]$ and $\pi\left(q_{j}^{(n)}\right)=\left[Q_{j}^{(n)}\right]$, these generators are in the image of $\pi$. Thus $\pi$ is surjective.

Since $\pi$ is surjective and the total composition is injective, it follows that $K_{0}(\eta)$ is injective. Finally, it is easy to see that $K_{0}(\eta)$ is surjective using Remark 5.

\section{Relation to Hilbert schemes}

We will now discuss how $\mathscr{H}^{\Gamma}$ acts on the derived categories of coherent sheaves on Hilbert schemes of points of the corresponding ALE space $X_{\Gamma}$. 
We take $\mathbb{k}=\mathbb{C}$. If $\Gamma \subset \mathrm{SL}_{2}(\mathbb{C})$ is finite, then by the McKay correspondence the derived category of finite-dimensional left $\left(\operatorname{Sym}^{*} V^{\vee} \rtimes \Gamma\right)$-modules is isomorphic to the derived category $D \operatorname{Coh}\left(X_{\Gamma}\right)$ of coherent sheaves on the resolution $X_{\Gamma}:=$ $\widehat{\mathbb{C}^{2} / \Gamma}$ of the quotient $\mathbb{C}^{2} / \Gamma$. To simplify notation we will denote the Hilbert scheme $\operatorname{Hilb}^{n}\left(X_{\Gamma}\right)$ by $X_{\Gamma}^{[n]}$.

Now by the results of Bridgeland, King, and Reid [2], the derived category $\ell_{n}^{\Gamma}$ of $A_{n}^{\Gamma}=\left(\mathrm{Sym}^{*} V^{\vee} \rtimes \Gamma\right)^{\otimes n} \rtimes S_{n}$ modules is isomorphic to the derived category of coherent sheaves on the $S_{n}$-equivariant Hilbert scheme $\operatorname{Hilb}^{S_{n}}\left(X_{\Gamma}^{n}\right)$. This Hilbert scheme parameterizes $S_{n}$-equivariant subschemes of $X_{\Gamma}^{n}$ whose global sections are isomorphic to the regular representation of $S_{n}$. By work of Haiman [13], Hilb ${ }^{S_{n}}\left(X_{\Gamma}^{n}\right)$ is isomorphic to the usual Hilbert scheme of points $X_{\Gamma}^{[n]}$.

Hence Theorem 2 gives a categorical Heisenberg action of $\mathscr{H}^{\Gamma}$ on $\bigoplus_{n} D \operatorname{Coh}\left(X_{\Gamma}^{[n]}\right)$. (If $\Gamma=\mathbb{C}^{\times}$, then we should think of $\mathcal{C}_{n}^{\Gamma}$ as the derived category of coherent sheaves on the Hilbert scheme of the quotient stack $\left[\mathbb{C}^{2} / \mathbb{C}^{\times}\right]$.)

We now explain how this action of $\mathscr{H}^{\Gamma}$ on $\bigoplus_{n} D \operatorname{Coh}\left(X_{\Gamma}^{[n]}\right)$ looks like geometrically. This description is to some degree conjectural because we only sketch why the algebraically defined functors $\mathrm{P}_{i}$ and $\mathrm{Q}_{i}$ from Section 4 induce the geometric functors defined in this section. Checking the relations in the 2-category $\mathscr{H}^{\Gamma}$ directly from the geometry (without using the algebraic description provided by Bridgeland, King, and Reid) is much more difficult. Two such computations are illustrated in Sections 8.1.1 and 8.1.2 below. These computations are analogous to those from [4] and [3] in the setting of categorical $\mathfrak{s l}_{n}$-actions. Because of the technical difficulties in such geometric setups, we chose to take a more algebraic approach in this paper.

\subsection{The functors}

Inside $X_{\Gamma}^{[n]} \times X_{\Gamma}^{[n+1]}$ we have the natural subvariety consisting of ideal sheaves $\left(J_{0}\right.$, $\left.J_{1}\right)$ where $J_{1} \subset J_{0}$. This subvariety also maps to $X_{\Gamma}$ by taking the quotient $J_{0} / J_{1}$ and subsequently induces a functor $D \operatorname{Coh}\left(X_{\Gamma}^{[n]} \times X_{\Gamma}\right) \rightarrow D \operatorname{Coh}\left(X_{\Gamma}^{[n+1]}\right)$. Under the correspondence

$$
D \operatorname{Coh}\left(X_{\Gamma}^{[n]}\right) \longleftrightarrow D\left(A_{n}^{\Gamma}-\bmod \right),
$$

this functor is induced by the $\left(A_{n+1}^{\Gamma}, A_{n}^{\Gamma} \times A_{1}^{\Gamma}\right)$-bimodule $A_{n+1}^{\Gamma}$ (where we use the usual inclusion of $A_{n}^{\Gamma} \otimes A_{1}^{\Gamma}$ into $A_{n+1}^{\Gamma}$ ).

Now, the fiber of $X_{\Gamma} \rightarrow \mathbb{C}^{2} / \Gamma$ over $0 \in \mathbb{C}^{2} / \Gamma$ is a tree of $\mathbb{P}^{1}$ 's. More precisely, there is one $E_{i} \cong \mathbb{P}^{1}$ for each $i \in I_{\Gamma}$, except for the node which corresponds to the trivial $\Gamma$-representation. (We label this node 0 and the corresponding minimal idempotent $e_{0}$.) Moreover, $E_{i}$ and $E_{j}$ intersect at a point if $i, j \in I_{\Gamma}$ are connected by an edge and do not intersect if $i, j$ are not connected. 
Under the McKay correspondence, the $A_{1}^{\Gamma}$-module $\mathbb{k}[\Gamma] e_{i}$ corresponds to the sheaf $\mathcal{O}_{E_{i}}(-1)$, while $\mathbb{k}[\Gamma] e_{0}$ corresponds to the structure sheaf $\mathcal{O}_{\cup} E_{i}[-1]$ shifted into cohomological degree 1 . We will use the notation $E_{0}:=\bigcup_{i} E_{i}$.

Now the functor $\mathrm{P}_{i}: \ell_{n}^{\Gamma} \rightarrow \bigodot_{n+1}^{\Gamma}$ in $\mathcal{H}^{\Gamma}$ is induced by $A_{n+1}^{\Gamma} \otimes_{A_{n}^{\Gamma} \otimes A_{1}^{\Gamma}}\left(A_{n}^{\Gamma} \otimes\right.$ $\left.\mathbb{k}[\Gamma] e_{i}\right)$. It should follow that the corresponding functor $\mathrm{P}_{i}: X_{\Gamma}^{[n]} \rightarrow X_{\Gamma}^{[n+1]}$ is induced by the kernels

$$
\mathcal{O}_{P_{i}(n, n+1)} \otimes \pi^{*} \mathcal{O}_{\mathbb{P}^{1}}(-1) \quad \text { if } i \neq 0 \quad \text { and } \quad \mathcal{O}_{P_{0}(n, n+1)}[-1] \quad \text { if } i=0 .
$$

Here

$$
P_{i}(n, n+1):=\left\{\left(J_{0}, J_{1}\right): \operatorname{supp}\left(J_{0} / J_{1}\right) \in E_{i}\right\} \subset X_{\Gamma}^{[n]} \times X_{\Gamma}^{[n+1]},
$$

and $\pi: P_{i}(n, n+1) \rightarrow E_{i}$ is the natural map which remembers the support point $\operatorname{supp}\left(J_{0} / J_{1}\right)$. The functors $\mathrm{Q}_{i}: X_{\Gamma}^{[n+1]} \rightarrow X_{\Gamma}^{[n]}$ are given by the adjoint of $\mathrm{P}_{i}$ (up to a shift).

The difference between the left and right adjoints of $\mathrm{P}_{i}$ is tensoring by the line bundle

$$
\pi_{1}^{*} \omega_{X_{\Gamma}^{[n]}}\left[\operatorname{dim} X_{\Gamma}^{[n]}\right] \otimes \pi_{2}^{*} \omega_{X_{\Gamma}^{[n+1]}}^{\vee}\left[-\operatorname{dim} X_{\Gamma}^{[n+1]}\right]
$$

Since $X_{\Gamma}$ is holomorphic symplectic, it follows that $X_{\Gamma}^{[n]}$ is also symplectic and hence has trivial canonical bundle. Thus (34) is the trivial line bundle with a shift of [-2]. (In other words, $\mathrm{P}_{i}^{L} \cong \mathrm{P}_{i}^{R}[-2]$.)

\subsubsection{The symmetric group}

To see the action of the symmetric group $S_{k} \subset \operatorname{End}\left(\mathrm{P}_{i}^{k}\right)$ we must first compute the convolution product of the kernels $\mathcal{O}_{P_{i}(n, n+1)} \otimes \pi^{*} \mathcal{O}_{\mathbb{P}^{1}}(-1)$. The line bundle $\pi^{*} \mathcal{O}_{\mathbb{P}^{1}}(-1)$ plays no role, so, for simplicity, we will omit it. The convolution $\mathcal{O}_{P_{i}(n+k-1, n+k)} * \cdots * \mathcal{O}_{P_{i}(n, n+1)} \in D \operatorname{Coh}\left(X_{\Gamma}^{[n]} \times X_{\Gamma}^{[n+k]}\right)$ is given by

$$
\pi_{0, k *}\left(\pi_{k-1, k}^{*} \mathcal{O}_{P_{i}(n+k-1, n+k)} \otimes \cdots \otimes \pi_{0,1}^{*} \mathcal{O}_{P_{i}(n, n+1)}\right),
$$

where $\pi_{i, j}$ is the projection $X_{\Gamma}^{[n+k]} \times \cdots \times X_{\Gamma}^{[n]} \rightarrow X_{\Gamma}^{[n+i]} \times X_{\Gamma}^{[n+j]}$. One can check that the intersections of $\pi_{l, l+1}^{-1} P_{i}(n+l, n+l+1)$ for $l=0, \ldots, k-1$ are of the expected dimension, and so (35) is isomorphic to $\pi_{0, k *}\left(\mathcal{O}_{\cap_{l} \pi_{l, l+1}^{-1} P_{i}(n+l, n+l+1)}\right)$. Now

$$
\begin{aligned}
& \bigcap_{l} \pi_{l, l+1}^{-1} P_{i}(n+l, n+l+1) \\
& \quad=\left\{J_{0} \supset J_{1} \supset \cdots \supset J_{k}: \operatorname{supp}\left(J_{l} / J_{l+1}\right) \in E_{i}\right\} \subset X_{\Gamma}^{[n]} \times \cdots \times X_{\Gamma}^{[n+k]} .
\end{aligned}
$$


The map $\pi_{0, k}$ forgets $J_{1}, \ldots, J_{k-1}$. Generically, $\left\{\operatorname{supp}\left(J_{l} / J_{l+1}\right): l=0, \ldots, k-1\right\}$ are $k$ distinct points on $E_{i}$. Thus $\pi_{0, k}$ is generically a cover of degree $k$ ! and there is a natural action of $S_{k}$ which permutes the points. This action should extend to all of $\bigcap_{l} \pi_{l, l+1}^{-1} P_{i}(n+l, n+l+1)$ and induce an action of $S_{k}$ on the pushforward in (35).

\subsubsection{The commutator relation}

It is also possible to geometrically see the commutator relation $Q_{i} P_{i} \cong P_{i} Q_{i} \oplus$ $\operatorname{id}[1] \oplus \operatorname{id}[-1]$. To do this let us compute the convolution

$$
\mathcal{O}_{P_{i}(n, n+1)} * \mathcal{O}_{P_{i}(n, n+1)} \in D \operatorname{Coh}\left(X_{\Gamma}^{[n]} \times X_{\Gamma}^{[n]}\right),
$$

which is the kernel corresponding to the composition $Q_{i} P_{i}$. (Again we ignore line bundles for convenience.) This is equal to

$$
\pi_{13}\left(\pi_{23}^{*} \mathcal{O}_{P_{i}(n, n+1)} \otimes \pi_{12}^{*} \mathcal{O}_{P_{i}(n, n+1)}\right) \cong \pi_{13 *}\left(\mathcal{O}_{\pi_{23}^{-1}\left(P_{i}(n, n+1)\right)} \otimes \mathcal{O}_{\pi_{12}^{-1}\left(P_{i}(n, n+1)\right)}\right) .
$$

Now

$$
\begin{aligned}
\pi_{23}^{-1} & P_{i}(n, n+1) \cap \pi_{12}^{-1} P_{i}(n, n+1) \\
\quad & =\left\{J_{0} \supset J_{1} \subset J_{0}^{\prime}: \operatorname{supp}\left(J_{0} / J_{1}, J_{0}^{\prime} / J_{1}\right) \in E_{i}\right\} \subset X_{\Gamma}^{[n]} \times X_{\Gamma}^{[n+1]} \times X_{\Gamma}^{[n]}
\end{aligned}
$$

has two components. One of them, denoted $\Delta_{0}$, is where $J_{0}=J_{0}^{\prime}$, while the other, denoted $\Delta_{1}$, is the closure of the locus where $J_{0} \neq J_{0}^{\prime}$. It is not hard to see that $\operatorname{dim}\left(\Delta_{1}\right)=2 n$, while $\operatorname{dim}\left(\Delta_{0}\right)=2 n+1$, which means that the intersection is of the expected dimension along $\Delta_{1}$ but not $\Delta_{0}$. The pushforward via $\pi_{13}$ is generically one-to-one along $\Delta_{1}$ and is a $\mathbb{P}^{1}$ fibration along $\Delta_{0}$.

Notice that $\pi_{13}\left(\Delta_{0}\right)$ is the diagonal $\Delta \subset X_{\Gamma}^{[n]} \times X_{\Gamma}^{[n]}$. Now one should show that $\Delta_{0}$ contributes $\mathcal{O}_{\Delta}[1] \oplus \mathcal{O}_{\Delta}[-1]$ via the pushforward. The computation here is a more involved version of the calculation

$$
\operatorname{Ext}_{X_{\Gamma}}^{k}\left(\mathcal{O}_{E_{i}}, \mathcal{O}_{E_{i}}\right) \cong \begin{cases}\mathbb{C} & \text { if } k=0,2, \\ 0 & \text { otherwise }\end{cases}
$$

occurring on the surface $X_{\Gamma}$ (which uses that the normal bundle of $E_{i} \cong \mathbb{P}^{1} \subset X_{\Gamma}$ is $\left.\mathcal{O}_{\mathbb{P}^{1}}(-2)\right)$.

On the other hand, $\Delta_{1}$ contributes just $\mathcal{O}_{\pi_{13}\left(\Delta_{1}\right)}$. Finally, one can check via a direct convolution computation (which is simpler than the one above) that $\mathcal{O}_{\pi_{13}\left(\Delta_{1}\right)}$ is actually equal to the kernel which induces $\mathrm{P}_{i} \mathrm{Q}_{i}$. Since $\mathcal{O}_{\Delta}$ induces the identity functor, the commutation relation follows.

\subsection{Nakajima's action on cohomology}

The fundamental class of the variety $P_{i}(n, n+1)$ induces one of Nakajima's Heisenberg operators on the cohomology of Hilbert schemes. The other Nakajima operators 
are given by fundamental classes of the varieties

$$
\begin{aligned}
P_{i}(n, n+k) & =\left\{\left(J_{0}, J_{1}\right): \operatorname{supp}\left(J_{0} / J_{1}\right) \text { is supported at a single point of } E_{i}\right\} \\
& \subset X_{\Gamma}^{[n]} \times X_{\Gamma}^{[n+k]} .
\end{aligned}
$$

Nakajima's theorem [19, Theorem 3.1] is that fundamental classes of the $P_{i}(n, n+k)$ (and their Poincaré duals) satisfy the defining relations of the "standard" Heisenberg generators $a_{i}(k)$ from Section 2. However, when $k>1$, the varieties $P_{i}(n, n+k)$ are singular, a fact which perhaps accounts for some of the difficulty of lifting the Nakajima action on cohomology to $K$-theory. In our categorical approach, the Heisenberg generators which are categorified are not the standard generators $a_{i}(k)$ but rather the generators $p_{i}^{(k)}$ which are categorified by the direct summands $\mathrm{P}_{i}^{(k)}$ of $\mathrm{P}_{i}^{k}$. In other words, $\mathrm{P}_{i}^{(k)}$ is the image of $e_{\text {triv }} \in S_{k} \subset \operatorname{End}\left(\mathrm{P}_{i}^{k}\right)$, where $e_{\text {triv }}$ is the idempotent inside $\mathbb{C}\left[S_{k}\right]$ corresponding to the trivial partition.

We do not know a direct, geometric description of the kernel which induces $\mathrm{P}_{i}^{(k)}$. The only geometry we can see is that involving $\mathrm{P}_{i}^{k}$ and the action of $S_{k}$ on it. One can probably guess the support of the kernel for $\mathrm{P}_{i}^{(k)}$, but it is going to be more complicated than just $P_{i}(n, n+k)$.

The difficulty in explicitly identifying $\mathrm{P}_{i}^{(k)}$ is probably related to the fact that it seems very hard to categorify the standard generators $a_{i}(k)$. By this we mean two things. First, we do not know if the $\mathcal{O}_{P_{i}(n, n+k)}$ induce an action of the standard generators $a_{i}(k)$ on derived categories of coherent sheaves. (We suspect that they do not.) Second, we do not know any categorical constructions of the expressions in Section 2.2 relating the generators $p_{i}^{(k)}$ and $a_{i}(k)$. Ideally, one would like some complex of functors involving $\mathrm{P}_{i}^{(k)}$ which categorify $a_{i}(k)$.

\section{Remark 7}

There is a graded version of this whole story, given by the equivalence

$$
D\left(A_{n}^{\Gamma} \text {-gmod }\right) \longleftrightarrow D \operatorname{Coh}^{\mathbb{C}^{\times}}\left(X_{\Gamma}^{[n]}\right),
$$

where the right-hand side is the derived category of $\mathbb{C}^{\times}$-equivariant sheaves. Here the action of $\mathbb{C}^{\times}$on $X_{\Gamma}^{[n]}$ is induced from the diagonal action of $\mathbb{C}^{\times}$on $\mathbb{C}^{2}$.

\section{An abelian 2-representation of $\mathscr{H}_{\Gamma}$}

We now describe another 2-representation of $\mathscr{H}_{\Gamma}$ which is closely related to the 2representation of Section 4 on $\bigoplus_{n} D\left(A_{n}^{\Gamma}\right.$-gmod $)$. More precisely, we replace $A_{n}^{\Gamma}$ with its Koszul dual

$$
B_{n}^{\Gamma}:=\left(B^{\Gamma} \otimes \cdots \otimes B^{\Gamma}\right) \rtimes S_{n} .
$$


(The algebra $B_{n}^{\Gamma}$ can also be written in smash product notation as $\left(B^{\Gamma}\right)^{\otimes n} \# \mathbb{k}\left[S_{n}\right]$.) The algebras $B_{n}^{\Gamma}$ are superalgebras, and it turns out that the Heisenberg category $\mathscr{H}_{\Gamma}$ acts on the module category $\bigoplus_{n} B_{n}^{\Gamma}$-gmod. Moreover, this action is nonderived and is therefore, in some sense, simpler than the action from Section 4. However, we have emphasized the action in Section 4 because of its closer relationship to geometry.

After recalling some basic definitions of superalgebras and modules over them, we will define all the generating 1- and 2-morphisms. The verification of relations, which is straightforward, will be left to the reader.

\subsection{Superalgebras and supermodules}

Let $B$ be a superalgebra. A left module over $B$ is a supervector space $W$, which is a left $B$-module in the usual sense. A right supermodule is a supervector space, which is a right $B$-module in the usual (nonsuper) sense, after ignoring the $\mathbb{Z}_{2}$-gradings on $B$ and on $W$.

Given two superalgebras $B_{1}$ and $B_{2}$, we form the tensor product superalgebra $B_{1} \otimes B_{2}$ with multiplication:

$$
(a \otimes b)\left(a^{\prime} \otimes b^{\prime}\right)=(-1)^{|b|\left|a^{\prime}\right|}\left(a a^{\prime} \otimes b b^{\prime}\right) .
$$

There is an isomorphism of superalgebras:

$$
B_{1} \otimes B_{2} \cong B_{2} \otimes B_{1}, \quad a \otimes b \mapsto(-1)^{|a||b|} b \otimes a .
$$

If $V$ and $W$ are left supermodules for $B_{1}$ and $B_{2}$, respectively, then $W_{1} \otimes W_{2}$ is a left supermodule for the superalgebra $B_{1} \otimes B_{2}$. The action of $B_{1} \otimes B_{2}$ on $W_{1} \otimes W_{2}$ is

$$
a \otimes b \cdot(v \otimes w)=(-1)^{|b||v|}(a \cdot v) \otimes(b \cdot w) .
$$

A morphism of left $B$-supermodules $f: W_{1} \rightarrow W_{2}$ is a map of vector spaces such that

$$
f(a \cdot v)=(-1)^{|f||a|} f(v), \quad v \in W_{1}, a \in B .
$$

Here $|f|$ is the degree of $f$ considered as a map of super vector spaces. In contrast, a morphism of right $B$-supermodules $g: W_{1} \rightarrow W_{2}$ is a map of vector spaces such that

$$
g(v \cdot a)=g(v) \cdot a, \quad v \in W_{1}, a \in B .
$$

Note that there is no sign here.

If $f: W_{1} \rightarrow W_{2}$ is a morphism of left $B_{1}$-supermodules and $g: W_{1}^{\prime} \rightarrow W_{2}^{\prime}$ is a morphism of left $B_{2}$-supermodules, then we write $f \otimes g$ as the induced morphism of left $\left(B_{1} \otimes B_{2}\right)$-modules 


$$
(f \otimes g): W_{1} \otimes W_{1}^{\prime} \longrightarrow W_{2} \otimes W_{2}^{\prime} .
$$

Explicitly, we have

$$
(f \otimes g)(v \otimes w)=(-1)^{|g||v|} f(v) \otimes g(w) .
$$

A $\left(B_{1}, B_{2}\right)$-superbimodule $W$ is a left $B_{1}$-supermodule which is a right $B_{2}$ supermodule for the same grading such that

$$
a \cdot(v \cdot b)=(a \cdot v) \cdot b .
$$

A morphism of superbimodules is a map which is a morphism of left and right supermodules. For the remainder of this section, all objects will be assumed super, though we will often omit writing the prefix "super" from discussions of algebras, modules, and bimodules.

\subsection{The algebras $B_{n}^{\Gamma}$}

Recall the algebra

$$
B_{n}^{\Gamma}:=\left(B^{\Gamma} \otimes \cdots \otimes B^{\Gamma}\right) \rtimes S_{n},
$$

which is the Koszul dual of $A_{n}^{\Gamma}$. Note that $B_{n}^{\Gamma}$ contains both $\mathbb{k}\left[S_{n}\right]$ and $B^{\Gamma \otimes n}$ as subalgebras, and the action of $S_{n}$ on $B^{\Gamma^{\otimes n}}$ is by superpermutations: if $s_{k} \in S_{n}$ is the simple transposition $(k, k+1)$, then

$$
\begin{aligned}
& s_{k} \cdot(\left.b_{1} \otimes \cdots \otimes b_{k} \otimes b_{k+1} \otimes \cdots \otimes b_{n}\right) \\
& \quad=(-1)^{\left|b_{k}\right|\left|b_{k+1}\right|} b_{1} \otimes \cdots \otimes b_{k+1} \otimes b_{k} \otimes \cdots \otimes b_{n} .
\end{aligned}
$$

\subsection{Functors $\mathrm{P}$ and $\mathrm{Q}$}

Consider the natural inclusion $B_{n}^{\Gamma} \hookrightarrow B_{n+1}^{\Gamma}$. We let $P^{\Gamma}(n)$ and $(n) Q^{\Gamma}$ denote $B_{n+1}^{\Gamma}\{1\}$ and $B_{n+1}^{\Gamma}$ thought of as a $\left(B_{n+1}^{\Gamma}, B_{n}^{\Gamma}\right)$-bimodule and $\left(B_{n}^{\Gamma}, B_{n+1}^{\Gamma}\right)$-bimodule, respectively.

Now $\mathbb{k}\left[S_{n+1}\right]$ is a flat $\mathbb{k}\left[S_{n}\right]$-module, where the module structure is defined via the natural inclusion $\mathbb{k}\left[S_{n}\right] \rightarrow \mathbb{k}\left[S_{n+1}\right]$. Subsequently, $B_{n+1}^{\Gamma}$ is a flat $B_{n}^{\Gamma}$-module. It follows that we can define the functors

$$
\begin{gathered}
\mathrm{P}(n): B_{n}^{\Gamma}-\operatorname{gmod} \longrightarrow B_{n+1}^{\Gamma}-\operatorname{gmod} \quad \text { and } \\
(n) \mathrm{Q}: B_{n+1}^{\Gamma}-\operatorname{gmod} \longrightarrow B_{n}^{\Gamma-\operatorname{gmod}}
\end{gathered}
$$

between abelian categories (instead of derived categories) using

$$
\mathrm{P}(n)(\cdot):=P^{\Gamma}(n) \otimes_{B_{n}^{\Gamma}}(\cdot) \quad \text { and } \quad(n) \mathrm{Q}(\cdot):=(n) Q^{\Gamma} \otimes_{B_{n+1}^{\Gamma}}(\cdot) .
$$

As before, we usually omit the $(n)$ to simplify notation. 


\subsection{Natural transformations}

\subsubsection{Definition of $X$}

For any $n \geq 1$ there is an inclusion $B_{1}^{\Gamma} \hookrightarrow B_{n}^{\Gamma}$ given by sending an element of $B_{1}^{\Gamma}$ to the last factor of the product

$$
a \mapsto(1 \otimes 1 \otimes \cdots \otimes 1 \otimes a) \in B_{n}^{\Gamma} .
$$

The image of this inclusion supercommutes with the subalgebra $B_{n-1}^{\Gamma} \subset B_{n}^{\Gamma}$. We henceforth consider $B_{1}^{\Gamma}$ as a subalgebra of $B_{n}^{\Gamma}$ via this embedding. For homogeneous $b \in B_{1}^{\Gamma}$ we define

$$
X(b): B_{n}^{\Gamma} \longrightarrow B_{n}^{\Gamma} w \mapsto(-1)^{|w||b|} w b
$$

using right multiplication by $b \in B_{1}^{\Gamma} \subset B_{n}^{\Gamma}$.

The map $X(b)$ is a map of $\left(B_{n}^{\Gamma}, B_{n-1}^{\Gamma}\right)$-bimodules. We thus get a well-defined natural transformation:

$$
X(b): \mathrm{P} \longrightarrow \mathrm{P}\{\operatorname{deg} b\}
$$

\subsubsection{Definition of $T$}

Notice that PP : $B_{n}^{\Gamma}-\operatorname{gmod} \rightarrow B_{n+2}^{\Gamma}-\operatorname{gmod}$ is induced by the $\left(B_{n+2}^{\Gamma}, B_{n}^{\Gamma}\right)$-bimodule

$$
B_{n+2}^{\Gamma}\{1\} \otimes_{B_{n+1}^{\Gamma}} B_{n+1}^{\Gamma}\{1\} \cong B_{n+2}^{\Gamma}\{2\} .
$$

So to define a degree 0 map, $T: \mathrm{PP} \rightarrow \mathrm{PP}$, we need a map $B_{n+2}^{\Gamma} \rightarrow B_{n+2}^{\Gamma}$ of $\left(B_{n+2}^{\Gamma}, B_{n}^{\Gamma}\right)$-bimodules. For this we use $w \mapsto w t_{n+1}$, where $t_{n+1}$ is the simple transposition $(n+1, n+2) \in S_{n+2}$. Since $t_{n+1}$ commutes with the subalgebra $B_{n}^{\Gamma} \subset$ $B_{n+2}^{\Gamma}$, this gives a well-defined map of $\left(B_{n+2}^{\Gamma}, B_{n}^{\Gamma}\right)$-bimodules.

\subsubsection{Definition of adj : QP $\rightarrow \operatorname{id}\{-1\}$}

The identity functor on $B_{n}^{\Gamma}-\operatorname{gmod}$ is given by tensoring with the $\left(B_{n}^{\Gamma}, B_{n}^{\Gamma}\right)$-bimodule $B_{n}^{\Gamma}$. On the other hand, QP is induced by the bimodule

$$
B_{n+1}^{\Gamma} \otimes_{B_{n+1}^{\Gamma}} B_{n+1}^{\Gamma}\{1\} .
$$

As a $\left(B_{n}^{\Gamma}, B_{n}^{\Gamma}\right)$-bimodule, this is generated by the subalgebra $B_{1}^{\Gamma}$ and the element $s_{n}$. (So any $\left(B_{n}^{\Gamma}, B_{n}^{\Gamma}\right)$-bimodule map is determined by where it sends $B_{1}^{\Gamma}$ and $s_{n}$.) We define a $\left(B_{n}^{\Gamma}, B_{n}^{\Gamma}\right)$-bimodule map,

$$
B_{n+1}^{\Gamma} \otimes_{B_{n+1}^{\Gamma}} B_{n+1}^{\Gamma} \rightarrow B_{n}^{\Gamma}\{-2\},
$$

by $s_{n} \mapsto 0,1 \mapsto 0, v_{1} \mapsto 0, v_{2} \mapsto 0$, and $\omega=v_{1} \wedge v_{2} \mapsto 1$, where $\left\{1, v_{1}, v_{2}, v_{1} \wedge v_{2}\right\}$ is the standard basis of $B_{1}^{\Gamma}$. This induces a map QP $\rightarrow \operatorname{id}\{-1\}$. 
9.4.4. Definition of adj $: P Q \rightarrow \operatorname{id}\{1\}$

The algebra multiplication

$$
B_{n+1}^{\Gamma} \otimes_{B_{n}^{\Gamma}} B_{n+1}^{\Gamma} \rightarrow B_{n+1}^{\Gamma} a \otimes b \mapsto a b
$$

is a map of $\left(B_{n+1}^{\Gamma}, B_{n+1}^{\Gamma}\right)$-bimodules and induces the map adj $: \mathrm{PQ} \longrightarrow \operatorname{id}\{1\}$.

\subsubsection{Definition of adj : id $\rightarrow \mathrm{PQ}\{1\}$}

The functor $\mathrm{PQ}\{1\}$ is induced by the $\left(B_{n+1}^{\Gamma}, B_{n+1}^{\Gamma}\right)$-bimodule $B_{n+1}^{\Gamma} \otimes_{B_{n}^{\Gamma}} B_{n+1}^{\Gamma}\{2\}$. Now any $\left(B_{n+1}^{\Gamma}, B_{n+1}^{\Gamma}\right)$-bimodule map from $B_{n+1}^{\Gamma}$ to this bimodule is determined by the image of $1 \in B_{n+1}^{\Gamma}$. Moreover, an element $b \in B_{n+1}^{\Gamma} \otimes_{B_{n}^{\Gamma}} B_{n+1}^{\Gamma}$ can be in the image of 1 under such a bimodule map if and only if $b$ is a Casimir element of $B_{n+1}^{\Gamma} \otimes_{B_{n}^{\Gamma}} B_{n+1}^{\Gamma}$ (i.e., if and only if $y b=(-1)^{|b||y|} b y$ for all $y \in B_{n+1}^{\Gamma}$ ).

We define the bimodule map $B_{n+1}^{\Gamma} \longrightarrow B_{n+1}^{\Gamma} \otimes_{B_{n}^{\Gamma}} B_{n+1}^{\Gamma}\{2\}$ by $1 \mapsto b_{0}$, where

$$
b_{0}=\sum_{b \in \mathscr{B}} \sum_{i=1}^{n+1} s_{i} \cdots s_{n} b^{\vee} \otimes b s_{n} \cdots s_{i},
$$

where $\mathcal{B}$ is our basis of $B_{1}^{\Gamma} \subset B_{n+1}^{\Gamma}$ and (by convention) $s_{i} \cdots s_{n}=s_{n} \cdots s_{i}=1$ when $i=n+1$ in the summation above. We leave it to the reader to check that $b_{0}$ is a Casimir element. This map induces adj : id $\rightarrow P Q\{1\}$.

\subsubsection{Definition of adj : id $\rightarrow$ QP $\{-1\}$}

The functor QP $\{-1\}$ is induced by the $\left(B_{n}^{\Gamma}, B_{n}^{\Gamma}\right)$-bimodule

$$
B_{n+1}^{\Gamma} \otimes_{B_{n+1}^{\Gamma}} B_{n+1}^{\Gamma} \cong B_{n+1}^{\Gamma} .
$$

The natural inclusion of $B_{n}^{\Gamma}$ as a subalgebra of $B_{n+1}^{\Gamma}$ is a map of $\left(B_{n}^{\Gamma}, B_{n}^{\Gamma}\right)$-bimodules and defines adj : id $\longrightarrow \mathrm{QP}\{-1\}$.

\subsection{The abelian 2-representation}

The following is the analogue of Theorem 2.

\section{THEOREM 3}

The functors $\mathrm{P}$ and $\mathrm{Q}$ together with natural transformations $X, T$, and adj defined above give a categorical action of $\mathcal{H}_{\Gamma}$ on the abelian category $\bigoplus_{n} B_{n}^{\Gamma}$-gmod.

The proof of the above theorem is analogous to the proof of Theorem 2, but easier, since none of the functors or natural transformations are derived. (In other words, there are no complexes involved and nowhere does one need to take resolutions.) 


\subsection{Relation to wreath products $\mathbb{k}\left[\Gamma^{n} \rtimes S_{n}\right]$}

The algebra $\mathbb{k}\left[\Gamma^{n} \rtimes S_{n}\right]$ embeds as a subalgebra (concentrated in degree 0 ) of $B_{n}^{\Gamma}$. This embedding gives rise to induction and restriction functors

$$
\begin{aligned}
& \operatorname{Ind}_{n}: \mathbb{k}\left[\Gamma^{n} \rtimes S_{n}\right]-\operatorname{gmod} \rightarrow B_{n}^{\Gamma}-\operatorname{gmod}, \\
& \operatorname{Res}_{n}: B_{n}^{\Gamma}-\operatorname{gmod} \rightarrow \mathbb{k}\left[\Gamma^{n} \rtimes S_{n}\right]-\operatorname{gmod}
\end{aligned}
$$

between categories of finite-dimensional (over $\mathbb{k}$ ) graded (super) modules. These functors are (up to shift) left and right adjoint to each other.

Denote by $K_{0}\left(\mathbb{k}\left[\Gamma^{n} \rtimes S_{n}\right]\right)$ and $K_{0}\left(B_{n}^{\Gamma}\right)$ the complexified Grothendieck groups of these categories, and denote by $\left.K_{P}\left(\mathbb{k}\left[\Gamma^{n} \rtimes S_{n}\right]\right]\right)$ and $K_{P}\left(B_{n}^{\Gamma}\right)$ the subgroups generated by projective modules. Of course, since the characteristic of $\mathbb{k}$ is zero, all $\mathbb{k}\left[\Gamma^{n} \rtimes S_{n}\right]$-modules are projective and $K_{0}\left(\mathbb{k}\left[\Gamma^{n} \rtimes S_{n}\right]\right)=K_{P}\left(\mathbb{k}\left[\Gamma^{n} \rtimes S_{n}\right]\right)$.

At the same time, $\mathbb{k}\left[\Gamma^{n} \rtimes S_{n}\right]$ is also a quotient of $B_{n}^{\Gamma}$ via the surjection which kills all elements of positive degree. This surjection induces an extension by a zero functor,

$$
\operatorname{ex}_{n}: \mathbb{k}\left[\Gamma^{n} \rtimes S_{n}\right]-\operatorname{gmod} \longrightarrow B_{n}^{\Gamma-g m o d},
$$

whose left adjoint is the functor

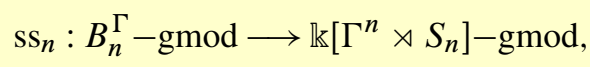

which takes a $B_{n}^{\Gamma}$-module to its maximal semisimple quotient considered naturally as a $\mathbb{k}\left[\Gamma^{n} \rtimes S_{n}\right]$-module. These functors have the following properties:

- $\quad$ the functors $\operatorname{Res}_{n}$ and $\operatorname{ex}_{n}$ induce isomorphisms $K_{0}\left(B_{n}^{\Gamma}\right) \cong K_{0}\left(\mathbb{k}\left[\Gamma^{n} \rtimes S_{n}\right]\right)$; - $\quad$ the functors $\operatorname{Ind}_{n}$ and $\mathrm{ss}_{n}$ induce isomorphisms $K_{P}\left(B_{n}^{\Gamma}\right) \cong K_{P}\left(\mathbb{k}\left[\Gamma^{n} \rtimes S_{n}\right]\right)$. The functor $\mathrm{ss}_{n}$ does not have finite homological dimension, so we restrict to the projective Grothendieck group in the second isomorphism. Therefore, as a corollary to Theorem 3, we obtain the following.

\section{COROLLARY 2}

The Heisenberg algebra $\mathfrak{h}_{\Gamma}$ acts on $\bigoplus_{n} K_{0}\left(\mathbb{k}\left[\Gamma^{n} \rtimes S_{n}\right]\right)$ and on $\bigoplus_{n} K_{P}\left(\mathbb{k}\left[\Gamma^{n} \rtimes S_{n}\right]\right)$.

This recovers a theorem of Frenkel, Jing, and Wang from [9, Theorem 6.1]. However, this action does not appear to come from a categorical action of $\mathscr{H}_{\Gamma}$ on the categories $\bigoplus_{n} \mathbb{k}\left[\Gamma^{n} \rtimes S_{n}\right]$ - gmod (in part because the 2-morphisms in $\mathscr{H}_{\Gamma}$ have nontrivial gradings). Indeed, the functors constructed in [9] give rise to a nondeformed Heisenberg algebra action (given by setting $t=1$ in the definition of $\mathfrak{h}_{\Gamma}$ ) on $\bigoplus_{n} K_{0}\left(\mathbb{k}\left[\Gamma^{n} \rtimes S_{n}\right]\right)$. 
In [10], Frenkel, Jing, and Wang obtain quantum Heisenberg actions by replacing $\Gamma$ with $\Gamma \times \mathbb{k}^{\times}$. This corresponds to considering graded versus ungraded $A_{n}^{\Gamma}$-modules in this section, as well as Section 4.

\section{Other remarks and open problems}

We would like to end by making some general remarks and discussing what we consider to be some interesting unanswered questions.

\subsection{Relationship to Khovanov's Heisenberg categorification and work of Shan and Vasserot}

Our 2-category $\mathscr{H}_{\Gamma}$ takes as its input data the embedded finite subgroup $\Gamma \subset \mathrm{SL}_{2}(\mathbb{C})$, and the definition of $\mathcal{H}_{\Gamma}$ can be generalized to an arbitrary embedded finite group $\Gamma \subset$ $\mathrm{SL}_{n}(\mathbb{C})$. The associated Heisenberg 2-category will then act naturally on

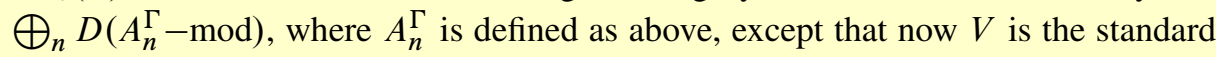
representation of $\mathrm{SL}_{n}(\mathbb{C})$. In fact, such a construction already makes sense when $\Gamma$ is trivial and $V=0$. In this case, the definition of the associated Heisenberg category is due to Khovanov [14].

In Khovanov's construction, like ours, the 1-morphisms are generated by two elements, $P$ and $Q$, which are biadjoint up to shift. The difference appears in the structure of 2-morphisms. Here the roles of $\Gamma$ and $V$ become apparent. In particular, our categories inherit a nontrivial $\mathbb{Z}$-grading from the natural grading on the exterior algebra $\Lambda^{*}(V)$. This grading makes the Grothendieck group of $\mathscr{H}_{\Gamma}$ into a $\mathbb{Z}\left[t, t^{-1}\right]$ module which categorifies a quantum deformation of the Heisenberg algebra.

It would be interesting to formulate a deeper relationship between our categorification and Khovanov's. One possible source for such a relationship comes from the representation theory of Hecke algebras at roots of unity. In particular, in [18] the second author, together with Alistair Savage, defined a $q$-deformation of Khovanov's Heisenberg categorification, recovering Khovanov's definition at $q=1$. At roots of unity, the Grothendieck group of the categorification of [18] changes. Moreover, if $q=e^{\frac{2 \pi i}{k}}$, the associated Heisenberg category acts on $\bigoplus_{n \geq 0} H_{n}(q)-\bmod$, where $H_{n}$ is the Hecke algebra of the symmetric group $S_{n}$. The category $\bigoplus_{n \geq 0} H_{n}(q)-\bmod$ was used earlier by Ariki [1] to categorify the basic representation of the affine Lie algebra $\widehat{\mathfrak{s t}_{k}}$. From this point of view, it is tempting to conjecturally identify Khovanov's Heisenberg categorification (after deforming as in [18] and setting $q=e^{\frac{2 \pi i}{k}}$ ) with a categorification of the principal Heisenberg subalgebra of the affine Lie algebra $\widehat{\mathfrak{s l}_{k}}$.

On the other hand, in [6] we describe a precise relationship between $\mathscr{H}^{\Gamma}$, where $\Gamma=\mathbb{Z} / k \mathbb{Z}$ is cyclic and the quantum affine algebra $\widehat{\mathfrak{s t}_{k}}$. Namely, $\mathscr{H}^{\Gamma}$ categorifies the homogeneous Heisenberg subalgebra of $\widehat{\mathfrak{s l}_{k}}$. Thus, at least in type A, a concrete 
relationship between our categorification and Khovanov's might come from the 2representation theory of the affine Lie algebra $\widehat{\mathfrak{s t}_{k}}$.

More recently, categorical Heisenberg actions have also appeared in the theory of cyclotomic rational double affine Hecke algebras (rDAHA). In [26], Shan and Vasserot describe certain induction and restriction functors between these rDAHAs, which are also defined using a cyclic subgroup $\Gamma \subset \mathrm{SL}_{2}(\mathbb{C})$. In fact, these rDAHAs are deformations of our algebras $A_{n}^{\Gamma}$, so the existence of a Heisenberg action should not be entirely surprising.

On the other hand, as far as we understand, Shan and Vasserot do not compute the categorical commutator relations between their $P$ and $Q$. (They only know the commutator relation at the level of Grothendieck groups.) Moreover, the type of Heisenberg they seem to get is a higher-level version of Khovanov's. This means that they have generators $P$ and $Q$ (but no $P_{i}$ and $Q_{i}$ for $i \in I_{\Gamma}$ ), with $[P, Q]$ equal to several copies of the identity.

After passing to the Grothendieck group, their Heisenberg corresponds to the diagonal Heisenberg inside $\widehat{\mathfrak{g l}}_{k}$, whereas in this paper we consider the homogeneous Heisenberg inside $\mathfrak{s \mathfrak { l }}_{k}$. These two combine to give the homogeneous Heisenberg inside $\widehat{\mathfrak{g l}_{k}}$. So it is likely that the construction in [26] is complementary to our work.

There is a large amount of work still to be done in this area, and we are happy to bring to the attention of the reader the problem of relating all the various categorical appearances of the Heisenberg algebra.

\subsection{Relation to Kac-Moody 2-categories $\mathcal{U}(\mathfrak{g})$}

When $\mathfrak{g}$ is the affine Lie algebra associated to $\Gamma$ we expect a direct relationship between the 2-categories $\mathscr{H}^{\Gamma}$ and $\mathcal{U}(\mathfrak{g})$. This expectation is motivated by the existence of two presentations of the affine Lie algebra $U(\mathfrak{g})$ - one as an extension of the loop algebra of the corresponding finite-dimensional Lie algebra and one as a KacMoody Lie algebra.

These two presentations should have two distinct categorifications. The 2categories $U(\mathfrak{g})$ from [17] and [24] categorify the Kac-Moody presentation. A second "loop categorification" should come from combining the 2-category associated with the finite-dimensional Lie algebra with our Heisenberg 2-category $\mathscr{H}^{\Gamma}$. One would like an equivalence between these categorifications, which lifts the isomorphism between the two different presentations. We will study such a relationship in [6].

\subsection{Degenerate affine Hecke algebras}

In $H^{\Gamma}$ we will also use hollow dots as shorthand for drawing right-twist curls. Subsequently, we obtain the following relations: 

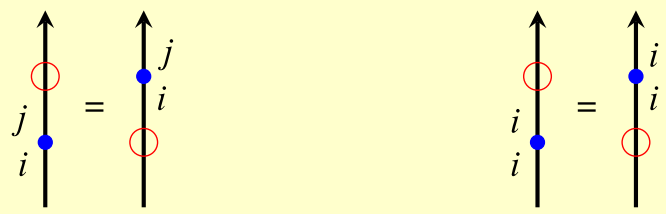

where $i, j \in I_{\Gamma}$ are joined by an edge. The hollow dots have an interesting "affine Hecke"-type relation with crossings:
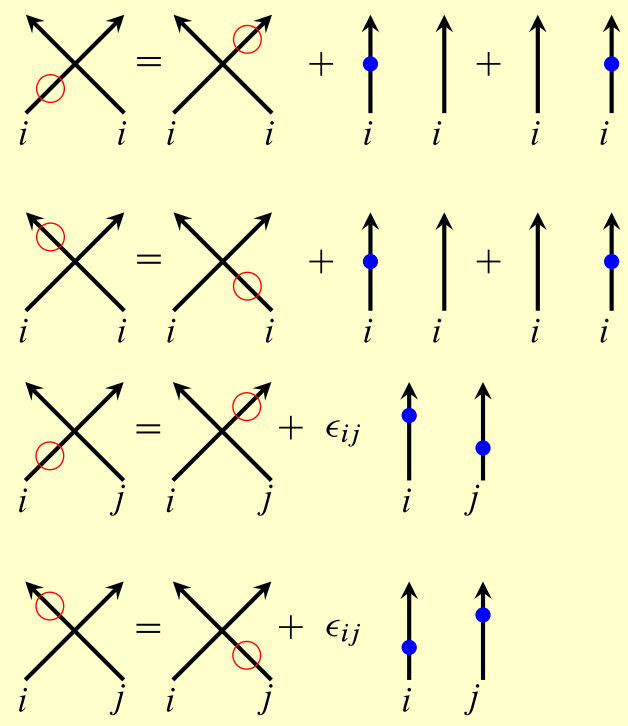

These relations are similar to the relations in the degenerate affine Hecke algebra of the symmetric group and its wreath products, which suggests that the endomorphism algebra of $P_{i}^{n}$ (or, more generally, the endomorphism algebra of $P_{i_{1}}^{n_{1}} \cdots P_{i_{k}}^{n_{k}}$ ) is an interesting algebraic object in its own right. It is perhaps worthwhile to express at least one of these algebras in more standard algebraic notation.

We thus define the graded $\mathbb{k}$-algebra $H_{i}^{n}$ as follows. The generators of $H_{i}^{n}$ are elements $y_{k}, z_{k}$ for $k=1, \ldots, n$ and $t_{l}$ for $1 \leq l \leq n-1$, where

$$
\operatorname{deg}\left(y_{k}\right)=\operatorname{deg}\left(z_{k}\right)=2 \quad \text { and } \quad \operatorname{deg}\left(t_{l}\right)=0 .
$$

The relations are as follows. First, the $\left\{t_{l}\right\}_{l=1}^{n-1}$ generate a subalgebra isomorphic to the group algebra $\mathbb{k}\left[S_{n}\right]$ of the symmetric group, with $t_{l}$ equal to the simple transposition $(l, l+1)$. The $\left\{y_{k}\right\}_{k=1}^{n}$ generate a symmetric algebra $\operatorname{Sym}\left(y_{1}, \ldots, y_{n}\right)$, and the $\left\{z_{k}\right\}_{k=1}^{n}$ generate an exterior algebra $\Lambda\left(z_{1}, \ldots, z_{n}\right)$. The remaining relations are

$$
y_{k} z_{l}=z_{l} y_{k} \quad \text { for all } k, l,
$$




$$
\begin{aligned}
t_{k} y_{l} & =y_{l} t_{k} \quad \text { for } l \neq k, k+1, \\
t_{k} z_{l} & =z_{l} t_{k} \quad \text { for } l \neq k, k+1, \\
t_{k} z_{k+1} & =z_{k} t_{k}, \\
t_{k} z_{k} & =z_{k+1} t_{k}, \\
t_{k} y_{k+1} & =y_{k} t_{k}+z_{k}+z_{k+1}, \\
y_{k+1} t_{k} & =t_{k} y_{k}+z_{k}+z_{k+1} .
\end{aligned}
$$

Note that there is a natural map of $\mathbb{k}$-algebras $H_{i}^{n} \longrightarrow \operatorname{End}_{\mathscr{H} \Gamma}\left(P_{i}^{n}\right)$ taking $t_{k}$ to a crossing between the $k$ and $(k+1)$ st strands, $y_{k}$ to a hollow dot on the $k$ th strand, and $z_{k}$ to a solid dot on the $k$ th strand.

\section{Conjecture 1}

The natural map of $\mathbb{k}$-algebras $H_{i}^{n} \longrightarrow \operatorname{End}_{\mathscr{H}^{\Gamma}}\left(P_{i}^{n}\right)$ is injective.

\section{Remark 8}

In Section 9 the functor $P^{n}$ is induced by the $\left(B_{k}^{\Gamma}, B_{k+n}^{\Gamma}\right)$-bimodule $B_{n+k}^{\Gamma}\{n\}$. Then it is clear from the above definitions of $X(a)$ and $T$ that the map $b \in B_{n}^{\Gamma}$ acts on $B_{n+k}^{\Gamma}\{n\}$ by right multiplication. Subsequently, the map $B_{n}^{\Gamma} \longrightarrow \operatorname{End}_{\mathscr{H}_{\Gamma}}\left(P^{n}\right)$ is injective. When $\Gamma$ is cyclic (i.e., when $\mathscr{H}_{\Gamma}$ and $\mathscr{H}^{\Gamma}$ agree), this means that the subalgebra of $H_{i}^{n}$ generated by the $t$ 's and $z$ 's injects into $\operatorname{End}_{\mathcal{H}} \Gamma\left(P_{i}^{n}\right)$.

The more complicated part of the conjecture above involves the hollow dots, which generate a commutative subalgebra of $\operatorname{End}_{\mathscr{H}} \Gamma\left(P^{n}\right)$. Under the analogy between $H_{i}^{n}$ and the degenerate affine Hecke algebra, it is natural to consider the image of hollow dots in representations of $\mathscr{H}^{\Gamma}$ as "Jucys-Murphy elements." We refer the reader to [14] for a related discussion where the algebra $B_{n}^{\Gamma}$ is replaced by the group algebra $\mathbb{k}\left[S_{n}\right]$ of the symmetric group, in which case the algebra $H_{i}^{n}$ is replaced by the degenerate affine Hecke algebra.

The space of 2-morphisms End(id) from the empty diagram to itself is spanned by closed diagrams. Moreover, since one can slide closed diagrams past vertical lines, one can assume that all closed components appear to the right of all components with boundary. On the other hand, any closed diagram acts naturally on any space of 2morphisms in $\mathscr{H}^{\Gamma}$, since closed diagrams can be added to the right of a 2-morphism to produce a new 2-morphism with the same source and target. A more ambitious version of Conjecture 1 is that, up to the action of End(id), all endomorphisms of $P_{i}^{n}$ come from the algebra $H_{i}^{n}$ : 


\section{Conjecture 2}

The natural map $H_{i}^{n} \otimes_{\mathbb{k}} \operatorname{End}_{\mathscr{H} \Gamma(\mathrm{id})} \longrightarrow \operatorname{End}_{\mathscr{H} \Gamma}\left(P_{i}^{n}\right)$ is an isomorphism.

\subsection{The structure of End $_{\mathscr{H} \Gamma}$ (id)}

Conjecture 2 reduces the problem of understanding $\operatorname{End}_{\mathscr{H} \Gamma}\left(P_{i}^{n}\right)$ to the problem of understanding $\operatorname{End}_{\mathscr{H} \Gamma}$ (id). An element of $\operatorname{End}_{\mathscr{H} \Gamma}$ (id) can be simplified to a linear combination of unions of disjoint, oriented circles containing solid and hollow dots. In fact, with a little manipulation one can also write any counterclockwise-oriented circle as a linear combination of products of clockwise-oriented circles.

Notice that if you have a circle labeled by $i \in I$ with a solid dot, then you can split the dot into two solid dots corresponding to maps $P_{i} \rightarrow P_{j}\langle 1\rangle \rightarrow P_{i}\langle 2\rangle$, where $i, j \in I$ are joined by an edge in the Dynkin diagram. Moving one of the dots around the other side and joining back the two dots gives a circle labeled $j$ with a solid dot. Thus it is clear that the label on a circle containing a solid dot is irrelevant.

For $i \in I$ and $k \in \mathbb{N}$,

- $\quad$ let $c_{k}^{i}$ be a clockwise circle labeled $i$ containing exactly $k$ hollow dots, and - $\quad$ let $c_{k}$ denote a clockwise circle containing one solid dot and $k$ hollow dots (the labeling of the circle $c_{k}$ is not important by the observation above).

Notice that $\operatorname{deg}\left(c_{k}^{i}\right)=2 k+2$ and $\operatorname{deg}\left(c_{k}\right)=2 k+4$.

\section{Conjecture 3}

End $_{\mathscr{H} \Gamma}$ (id) is isomorphic to the symmetric algebra freely generated by $\left\{c_{k}^{i}\right\}_{k \geq 0, i \in I}$ and $\left\{c_{k}\right\}_{k \geq 0}$.

\subsection{Hochschild cohomology of Hilbert schemes}

Let us work over $\mathbb{k}=\mathbb{C}$. Our categorical action of $\mathscr{H}^{\Gamma}$ on $\bigoplus_{n} D \operatorname{Coh}\left(X_{\Gamma}^{[n]}\right)$ gives a degree-preserving map of rings,

$$
\operatorname{End}_{\mathscr{H} \Gamma}\left(\operatorname{id}_{n}\right) \rightarrow H H^{*}\left(X_{\Gamma}^{[n]}\right),
$$

where $\mathrm{id}_{n}$ is the identity functor of the region labeled by $n \in \mathbb{Z}$ and where $H H^{*}$ denotes the Hochschild cohomology. Unfortunately, this map is neither injective nor surjective. For example, $H H^{1}\left(X_{\Gamma}\right)$ is nonzero because $X_{\Gamma}$ carries nontrivial vector fields, but $\operatorname{End}_{\mathscr{H}^{\Gamma}}\left(\operatorname{id}_{n}\right)$ is zero in odd degrees.

On the other hand, one can consider "cyclotomic quotients" of $\operatorname{End}_{\mathscr{H} \Gamma}\left(\operatorname{id}_{n}\right)$ much like those of quiver Hecke algebras considered in [15], [16], [17], and [24]. For example, one such quotient, $\overline{\operatorname{End}}_{\mathscr{H}} \Gamma\left(\mathrm{id}_{n}\right)$, is defined as the quotient by the ideal generated by diagrams which contain a region labeled by an integer $n<0$. 


\section{Example}

$\overline{\text { End }}_{\mathscr{H}} \Gamma\left(\mathrm{id}_{0}\right) \cong \mathbb{C}$ essentially because any counterclockwise circle is zero or the identity and any other diagram will contain a region labeled by some $n<0$.

\section{Example}

Likewise, we have

$$
\overline{\operatorname{End}}_{\mathscr{H} \Gamma}\left(\mathrm{id}_{1}\right) \cong \begin{cases}\mathbb{C} & \text { in degree } 0,4, \\ \mathbb{C}^{|\Gamma|} & \text { in degree } 2, \\ 0 & \text { in all other degrees, }\end{cases}
$$

where the degree 2 maps are spanned by clockwise circles labeled by some $i \in I_{\Gamma}$. The product of any two such circles is equal to a clockwise circle containing a solid dot. (This spans the space of degree 4 maps.)

The map (37) factors through $\overline{\mathrm{End}}_{\mathscr{H}_{\Gamma}}\left(\mathrm{id}_{n}\right)$. One would like to know if the resulting morphism has any interesting properties. Likewise, one can ask this same question if you replace $D \operatorname{Coh}\left(X_{\Gamma}^{[n]}\right)$ by the category $B_{n}^{\Gamma}-\operatorname{gmod}$ from Section 9.

\section{Appendix. The case $\Gamma=\mathbb{Z}_{2}$}

The case $\Gamma=\mathbb{Z}_{2}$ differs slightly from other nontrivial $\Gamma \subset \mathrm{SL}_{2}(\mathbb{C})$, and the main definitions in the body of the paper need to be modified slightly. We collect the appropriate definitions for $\Gamma=\mathbb{Z}_{2}$ in this section. Having made these definitions, all of the results and proofs in the body of the paper carry over to $\Gamma=\mathbb{Z}_{2}$ without difficulty.

The vertex set $I_{\mathbb{Z}_{2}}=\{0,1\}$ consists of the two distinct isomorphism classes of representations of $\mathbb{Z}_{2}$, the trivial representation and the sign representation. The quantum Cartan matrix $C_{\mathbb{Z}_{2}}$ is the matrix with entries $\langle i, j\rangle$ indexed by $i, j \in I_{\mathbb{Z}_{2}}$ given by

$$
\langle i, j\rangle= \begin{cases}t+t^{-1} & \text { if } i=j, \\ -2 & \text { if } i \neq j .\end{cases}
$$

The Heisenberg algebra $\mathfrak{h}_{\mathbb{Z}_{2}}$ is the unital infinite-dimensional $\mathbb{k}\left[t, t^{-1}\right]$-algebra with generators $p_{i}^{(n)}, q_{i}^{(n)}$ for $i \in I_{\mathbb{Z}_{2}}$ and integers $n \geq 0$, with relations

$$
\begin{array}{ll}
p_{i}^{(n)} p_{j}^{(m)}=p_{j}^{(m)} p_{i}^{(n)} \quad \text { and } \quad q_{i}^{(n)} q_{j}^{(m)}=q_{j}^{(m)} q_{i}^{(n)} & \text { for any } i, j \in I_{\mathbb{Z}_{2}} ; \\
q_{i}^{(n)} p_{j}^{(m)}= \begin{cases}\sum_{k \geq 0}[k+1] p_{i}^{(m-k)} q_{i}^{(n-k)} & \text { if } i=j, \\
p_{j}^{(m)} q_{i}^{(n)}+2 p_{j}^{(m-1)} q_{i}^{(n-1)}+p_{j}^{(m-2)} q_{i}^{(n-2)} & \text { if } i \neq j .\end{cases}
\end{array}
$$

The definition of the 2-category $\mathscr{H}_{\mathbb{Z}_{2}}^{\prime}$ is the same as before (and $\mathscr{H}_{\mathbb{Z}_{2}}$ is still defined as the Karoubi envelope of $\mathscr{H}_{\mathbb{Z}_{2}}^{\prime}$ ). 
On the other hand, $\mathscr{H}^{\prime \mathbb{Z}_{2}}$ is slightly different. We fix an orientation $\epsilon$ of the Dynkin diagram. Since there are two edges connecting the two vertices $i$ and $j$, we describe the orientation $\epsilon$ by two functions, $\epsilon_{i j}(0)$ and $\epsilon_{i j}(1)$. More precisely, $\epsilon_{i j}(0)= \pm 1$ depending on whether the first edge is oriented from $i$ to $j$ or from $j$ to $i$. Similarly, $\epsilon_{i j}(1)$ describes the orientation of the second edge.

As in the case $\Gamma \neq \mathbb{Z}_{2}$, the objects of $\mathscr{H}^{\prime \mathbb{Z}_{2}}$ are indexed by the integers $\mathbb{Z}$, while the 1-morphisms are generated by $P_{i}$ and $Q_{i}\left(i \in I_{\mathbb{Z}_{2}}\right)$.

The space of 2-morphisms is the $\mathbb{k}$-algebra generated by suitable planar diagrams modulo local relations. As before, the diagrams consist of oriented compact 1 -manifolds immersed into the plane strip $\mathbb{R} \times[0,1]$ (possibly with crossings) modulo isotopies fixing the boundary.

Each such strand is labeled by some $i \in I_{\mathbb{Z}_{2}}$. It can carry a solid dot as before, which represents a map $X: P_{i} \rightarrow P_{i}\langle 2\rangle$. These $i i$ dots move freely on strands and through crossings just as in the case $\Gamma \neq \mathbb{Z}_{2}$.

The main difference is that the strands now also carry a hollow rectangle (see below). More precisely, corresponding to each of the two edges in the Dynkin diagram connecting vertices $i$ and $j$ there is a 2-morphism $X: P_{i} \rightarrow P_{j}\langle 1\rangle$ (one denoted by the solid dot and the other by the hollow rectangle):

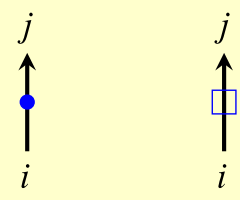

These dots and rectangles are also allowed to move freely along the 1-manifold along cups and caps and through crossings. Dots or rectangles on distinct strands anticommute when they move past each other relative to the vertical. The relations which govern compositions are as follows:
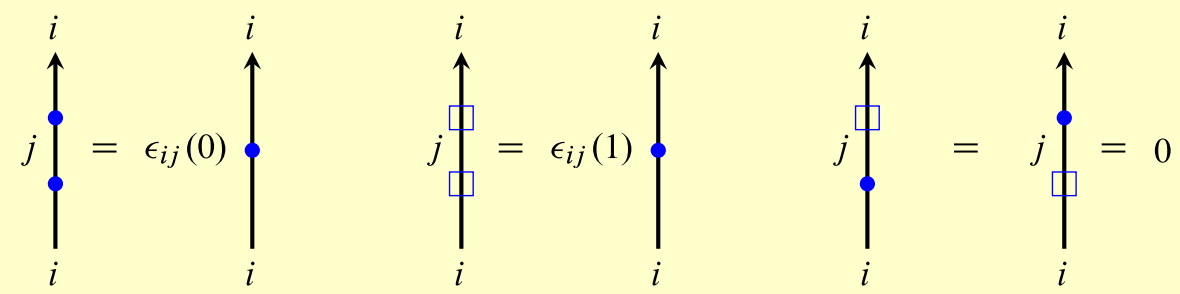

Also, the composition of an $i i$ dot with any other dot or rectangle is zero.

Next, we have the same relations (27) and (28), as well as (30). The only more interesting relation is (29), which is replaced by 


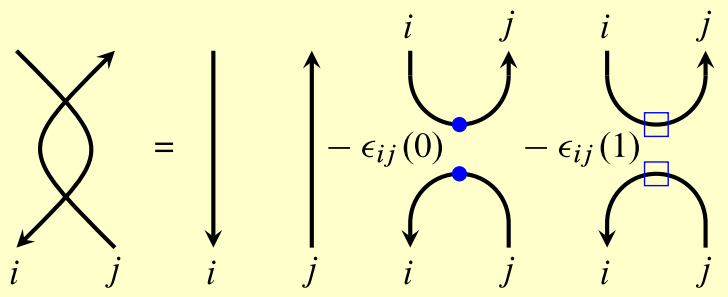

Finally, we assign a $\mathbb{Z}$-grading on the space of planar diagrams like before. In particular, we define the degree of both an $i j$ dot and an $i j$ rectangle to be one when $i \neq j$ and the degree of an $i i$ dot to be two. Equipped with these assignments, all the graphical relations are graded, providing a $\mathbb{Z}$-grading on $\mathscr{H}^{\prime \mathbb{Z}_{2}}$. We then define $\mathscr{H}^{\mathbb{Z}_{2}}$ to be the Karoubi envelope of $\mathscr{H}^{\prime \mathbb{Z}_{2}}$.

Acknowledgments. The authors would like to thank Mikhail Khovanov for several useful conversations and for giving us an early version of his paper [14]. Much of this paper is inspired by his work. We would also like to thank Igor Frenkel for sharing with us his unpublished notes with Khovanov and Malkin on categorification and vertex operator algebras [11], and Alistair Savage for useful comments regarding an earlier version of this paper. This project began when both authors were at the program "Homology Theory of Knots and Links" at the Mathematical Sciences Research Institute (MSRI) in 2010. We thank MSRI for their hospitality and great working environment.

\section{References}

[1] S. ARIKI, "Lectures on cyclotomic Hecke algebras" in Quantum Groups and Lie Theory (Durham, 1999), London Math. Soc. Lecture Note Ser. 290, Cambridge Univ. Press, Cambridge, 2001, 1-22. MR 1903956 (2538)

[2] T. BRIDGELAND, A. KING, and M. REID, The McKay correspondence as an equivalence of derived categories, J. Amer. Math. Soc. 14 (2001), 535-554. MR 1824990 (2529)

[3] S. CAUTIS and J. KAMNITZER, Braiding via geometric Lie algebra actions, Compos. Math. 148 (2012), 464-506. MR 2904194 (2529)

[4] S. CAUTIS, J. KAMNITZER, and A. LICATA, Categorical geometric skew Howe duality, Invent. Math. 180 (2010), 111-159. MR 2593278 (2529)

[5] Coherent sheaves on quiver varieties and categorification, preprint, arXiv:1104.0352v1 [math.AG] (2471)

[6] S. CAUTIS and A. LICATA, Vertex operators and 2-representations of quantum affine algebras, preprint, arXiv:1112.6189v1 [math.RT] (2538, 2539)

[7] N. CHRISS and V. GINZBURG, Representation Theory and Complex Geometry, Birkhäuser, Boston, 1997. MR 1433132 (2470) 
[8] B. FEIGIN and A. TSYMBALIUK, Equivariant K-theory of Hilbert schemes via shuffle algebra, Kyoto J. Math. 51 (2011), 831-854. MR 2854154 (2469, 2470)

[9] I. FRENKEL, N. JING, and W. WANG, Vertex representations via finite groups and the McKay correspondence, Int. Math. Res. Not. IMRN 2000, no. 4, 195-222. MR 1747618 (2471, 2472, 2528, 2537)

[10] - Quantum vertex representations via finite groups and the McKay correspondence, Comm. Math. Phys. 211 (2000), 365-393. MR 1754520 (2472, 2498, 2528, 2538)

[11] I. FRENKEL, M. KHOVANOV, and A. MALKIN, unpublished notes, circa 2003. (2545)

[12] I. GROJNOWSKI, Instantons and affine Lie algebras I: The Hilbert scheme and vertex operators, Math. Res. Lett. 3 (1996), 275-291. MR 1386846 (2469, 2471, 2472)

[13] M. HAIMAN, Hilbert schemes, polygraphs and the Macdonald positivity conjecture, J. Amer. Math. Soc. 14 (2001), 941-1006. MR 1839919 (2529)

[14] M. KHOVANOV, Heisenberg algebra and a graphical calculus, preprint, arXiv:1009.3295v1 [math.RT] $(2538,2541,2545)$

[15] M. KHOVANOV and A. LAUDA, A diagrammatic approach to categorification of quantum groups, I, Represent. Theory 13 (2009), 309-347. MR 2525917 (2470, 2477, 2480, 2542)

[16] A diagrammatic approach to categorification of quantum groups, II, Trans. Amer. Math. Soc. 363 (2011), 2685-2700. MR 2763732 (2470, 2471, 2477, 2480, 2542)

[17] - A categorification of quantum $\mathrm{sl}(n)$, Quantum Topol. 1 (2010), 1-92. MR 2628852 (2470, 2477, 2480, 2539, 2542)

[18] A. LICATA and A. SAVAGE, Hecke algebras, finite general linear groups, and Heisenberg categorification, to appear in Quantum Topol., preprint, arXiv:1101.0420v2 [math.RT] (2538)

[19] H. NAKAJIMA, Heisenberg algebra and Hilbert schemes of points on projective surfaces, Ann. of Math. (2) 145 (1997), 379-388. MR 1441880 (2469, 2471, 2472, 2532)

[20] - Quiver varieties and Kac-Moody algebras, Duke Math. J. 91 (1998), 515-560. MR 1604167 (2470)

[21] - Lectures on Hilbert Schemes of Points on Surfaces, Univ. Lecture Ser. 18, Amer. Math. Soc., Providence, 1999. MR 1711344 (2475)

[22] - Quiver varieties and finite-dimensional representations of quantum affine algebras, J. Amer. Math. Soc. 14 (2001), 145-238. MR 1808477 (2470)

[23] A. RAM and A. SHEPLER, Classification of graded Hecke algebras for complex reflection groups, Comment. Math. Helv. 78 (2003), 308-334. MR 1988199 (2487)

[24] R. ROUQUIER, 2-Kac-Moody algebras, preprint, arXiv:0812.5023v1 [math.RT] (2470, 2477, 2480, 2539, 2542)

[25] O. SCHIFFMANN and E. VASSEROT, The elliptic Hall algebra and the K-theory of the Hilbert scheme of $\mathbb{A}^{2}$, preprint, arXiv:0905.2555v3 [math.QA] $(2469,2470)$

[26] P. SHAN and E. VASSEROT, Heisenberg algebras and rational double affine Hecke algebras, J. Amer. Math. Soc. 25 (2012), 559-1031. MR 2947944 (2539) 
[27] J. WAN, Wreath Hecke algebras and centralizer construction for wreath products, J. Algebra 323 (2010), 2371-2397. MR 2602385 (2487)

[28] J. WAN and W. WANG, Modular representations and branching rules for wreath Hecke algebras, Int. Math. Res. Not. IMRN 2008, art. ID rnn128. MR 2449051 (2487)

\section{Cautis}

Department of Mathematics, Columbia University, New York, New York 10027, USA; scautis@math.columbia.edu

\section{Licata}

Department of Mathematics, Stanford University, Stanford, California 94305, USA; amlicata@math.stanford.edu 